University of Tennessee Health Science Center UTHSC Digital Commons

$12-2018$

\title{
Roles of Cytosolic Nucleic Acid Sensors in Cancer and Infection
}

Qifan Zhu

University of Tennessee Health Science Center

Follow this and additional works at: https://dc.uthsc.edu/dissertations

Part of the Biological Phenomena, Cell Phenomena, and Immunity Commons, Medical Cell Biology Commons, and the Medical Immunology Commons

\section{Recommended Citation}

Zhu, Qifan (http://orcid.org/0000-0001-7174-7943), "Roles of Cytosolic Nucleic Acid Sensors in Cancer and Infection" (2018). Theses and Dissertations (ETD). Paper 477. http://dx.doi.org/10.21007/ etd.cghs.2018.0462.

This Dissertation is brought to you for free and open access by the College of Graduate Health Sciences at UTHSC Digital Commons. It has been accepted for inclusion in Theses and Dissertations (ETD) by an authorized administrator of UTHSC Digital Commons. For more information, please contact jwelch30@uthsc.edu. 


\title{
Roles of Cytosolic Nucleic Acid Sensors in Cancer and Infection
}

\begin{abstract}
Pattern recognition receptors are innate immune sensors that recognize pathogen-associated molecular patterns (PAMPs) and danger-associated molecular patterns (DAMPs) with crucial roles in host defense against microbial infection, autoimmune diseases and cancer. Cytosolic nucleic acids including DNA and RNA originate from pathogens or self-cells, which form major groups of PAMPs and DAMPs. A range of nucleic acid sensors have evolved to sense various types of nucleic acids. How different DNA-sensing pathways regulate microbial infection and cancer is the focus of this dissertation.
\end{abstract}

Stimulator of IFN genes (STING) is a cytosolic innate immune sensor for cyclic dinucleotides that also serves a dual role as an adaptor molecule for a number of intracellular DNA receptors. A physiological role for STING in cancer was previously unknown. We showed that STING-deficient mice were highly susceptible to colitis-associated colorectal cancer. Colons of STING-deficient mice exhibited significant intestinal damage and overt proliferation with increased levels of pro-inflammatory cytokines during early stages of tumorigenesis, uncovering an unexpected and important role for STING in mediating protection against colorectal tumorigenesis.

Absent in melanoma 2 (AIM2) forms an inflammasome with ASC and caspase- 1 upon recognition of double-stranded DNA (dsDNA) in the cytosol leading to caspase-1 activation and caspase-1-dependent pyroptosis and release of cytokines IL-10 and IL-18. Mutations in AIM2 are frequently identified in patients with colorectal cancer, but how AIM2 modulates colonic tumorigenesis is unknown. We found that AIM2-deficient mice were hypersusceptible to colonic tumor development. While production of inflammasome-associated cytokines and other inflammatory mediators was largely intact in AIM2-deficient mice, intestinal stem cells lacking AIM2 were prone to uncontrolled proliferation. Aberrant Wnt signaling expanded a population of tumor-initiating stem cells in the absence of AIM2 driving the tumor development in AIM2-deficient mice. In addition to its role in cancer, AIM2 recognizes bacteria including Francisella tularensis subspecies novicida (F. novicida) and induces inflammasome responses. Type I interferon (IFN) signaling drives activation of AIM2 inflammasome in F. novicida-infected macrophages; however, the relative contribution of IFNs and inflammasome responses in host defense against $F$. novicida infection is less understood. We found intact AIM2 inflammasome responses in mice lacking type I IFN signaling during infection with F. novicida. Lack of type I IFN signaling conferred protection to $F$. novicida infection in contrast to the increased susceptibility in AIM2-deficient mice. Interestingly, mice lacking both AIM2 and IFNAR2 were protected against the infection indicating a dominant role for type I IFNs in mediating detrimental responses despite the protective AIM2 inflammasome responses. Gasdermin D (GSDMD) is activated by caspase- 1 to generate pores on the plasma membrane to induce pyroptosis downstream of the AIM2 inflammasome. We also demonstrated that mice lacking GSDMD were highly susceptible to $F$. novicida infection. Interestingly, GSDMD is required for optimal caspase-1 activation during F. novicida infection, providing protection to the host during the infection.

In addition, we identified differential mechanisms regulating expression of inflammasome-associated cytokines IL-1 $\beta$ and IL-18. IL-1 $\beta$ is only induced in response to inflammatory stimuli and its expression is not sustained during chronic treatment, while IL-18 is constitutively expressed and further induced after the stimulation in a type I IFN signaling-dependent manner.

Overall, this dissertation addresses protective roles for cytosolic nucleic acid-sensing molecules, STING and AIM2, in colon cancer, uncovers an interplay between the AIM2 inflammasome and type IFN signaling during $F$. novicida infection, and demonstrates a novel function for GSDMD in regulating AIM2 inflammasome. The dissertation finally describes distinctive mechanisms governing inflammasome- 
associated cytokines IL-1 $\beta$ and IL-18.

Document Type

Dissertation

Degree Name

Doctor of Philosophy (PhD)

Program

Biomedical Sciences

Research Advisor

Thirumala-Devi Kanneganti, Ph.D.

Keywords

Cancer, Immunology, Infection, Inflammasome, Innate immunity, Pattern recognition receptors

Subject Categories

Biological Phenomena, Cell Phenomena, and Immunity | Medical Cell Biology | Medical Immunology | Medical Sciences | Medicine and Health Sciences 


\title{
Roles of Cytosolic Nucleic Acid Sensors in Cancer and Infection
}

\author{
A Dissertation \\ Presented for \\ The Graduate Studies Council \\ The University of Tennessee \\ Health Science Center
}

\author{
In Partial Fulfillment \\ Of the Requirements for the Degree \\ Doctor of Philosophy \\ From The University of Tennessee
}

By

Qifan Zhu

December 2018 
Chapter 2 and 3 (C) 2014 by American Association of Immunologists, Inc. Chapter 2 and 7 (C) 2017 by American Association of Immunologists, Inc.

All other materials (C) 2018 by Qifan Zhu All rights reserved. 


\section{ACKNOWLEDGEMENTS}

I would like to express my greatest gratitude to my advisor Dr. Thirumala-Devi Kanneganti for allowing me to complete the graduate training and for her guidance and support. Her enthusiasm on science and critical mind on research greatly inspired me during my Ph.D. study. I want to thank all the previous and current lab members of the Kanneganti lab for their continuous support. I also would like to thank my graduate committee members, Drs. Elizabeth Fitzpatrick, Gerard Zambetti, Hongbo Chi, Maureen McGargill and Terrence Geiger. I want to acknowledge my collaborators, Drs. Liqin Zhu, Liyuan Li, Richard J. Gilbertson (now at University of Cambridge), Geoffrey Neale, Scott R. Olsen, Daniel J. McGoldrick, David Finkelstein, Gang Wu, Robert A. Carter from St. Jude Children's Research Hospital and Dr. Mohamed Lamkanfi from (now at Janssen Immunosciences) from Vlaams Instituut voor Biotechnologie and Ghent University. I also want to acknowledge the Veterinary Pathology Core and the Flow cytometry core from St. Jude Children's Research Hospital.

I would like to thank my wife Huiyi Yang for her company and all my family members for their support. I want to thank my friends that I have met during the Ph.D. period.

The studies are funded by National Institutes of Health Grants (AR056296, CA163507, AI124346 and AI101935), the American Lebanese Syrian Associated Charities, European Research Council Grant (281600), and the Fund for Scientific Research-Flanders (G030212N, 1.2.201.10.N.00, and 1.5.122.11.N.00). 


\begin{abstract}
Pattern recognition receptors are innate immune sensors that recognize pathogenassociated molecular patterns (PAMPs) and danger-associated molecular patterns (DAMPs) with crucial roles in host defense against microbial infection, autoimmune diseases and cancer. Cytosolic nucleic acids including DNA and RNA originate from pathogens or self-cells, which form major groups of PAMPs and DAMPs. A range of nucleic acid sensors have evolved to sense various types of nucleic acids. How different DNA-sensing pathways regulate microbial infection and cancer is the focus of this dissertation.
\end{abstract}

Stimulator of IFN genes (STING) is a cytosolic innate immune sensor for cyclic dinucleotides that also serves a dual role as an adaptor molecule for a number of intracellular DNA receptors. A physiological role for STING in cancer was previously unknown. We showed that STING-deficient mice were highly susceptible to colitisassociated colorectal cancer. Colons of STING-deficient mice exhibited significant intestinal damage and overt proliferation with increased levels of pro-inflammatory cytokines during early stages of tumorigenesis, uncovering an unexpected and important role for STING in mediating protection against colorectal tumorigenesis.

Absent in melanoma 2 (AIM2) forms an inflammasome with ASC and caspase-1 upon recognition of double-stranded DNA (dsDNA) in the cytosol leading to caspase-1 activation and caspase-1-dependent pyroptosis and release of cytokines IL-1 $\beta$ and IL-18. Mutations in AIM2 are frequently identified in patients with colorectal cancer, but how AIM2 modulates colonic tumorigenesis is unknown. We found that AIM2-deficient mice were hypersusceptible to colonic tumor development. While production of inflammasome-associated cytokines and other inflammatory mediators was largely intact in AIM2-deficient mice, intestinal stem cells lacking AIM2 were prone to uncontrolled proliferation. Aberrant Wnt signaling expanded a population of tumor-initiating stem cells in the absence of AIM2 driving the tumor development in AIM2-deficient mice. In addition to its role in cancer, AIM2 recognizes bacteria including Francisella tularensis subspecies novicida (F. novicida) and induces inflammasome responses. Type I interferon (IFN) signaling drives activation of AIM2 inflammasome in $F$. novicidainfected macrophages; however, the relative contribution of IFNs and inflammasome responses in host defense against $F$. novicida infection is less understood. We found intact AIM2 inflammasome responses in mice lacking type I IFN signaling during infection with $F$. novicida. Lack of type I IFN signaling conferred protection to $F$. novicida infection in contrast to the increased susceptibility in AIM2-deficient mice. Interestingly, mice lacking both AIM2 and IFNAR2 were protected against the infection indicating a dominant role for type I IFNs in mediating detrimental responses despite the protective AIM2 inflammasome responses. Gasdermin D (GSDMD) is activated by caspase-1 to generate pores on the plasma membrane to induce pyroptosis downstream of the AIM2 inflammasome. We also demonstrated that mice lacking GSDMD were highly susceptible to $F$. novicida infection. Interestingly, GSDMD is required for optimal 
caspase- 1 activation during $F$. novicida infection, providing protection to the host during the infection.

In addition, we identified differential mechanisms regulating expression of inflammasome-associated cytokines IL-1 $\beta$ and IL-18. IL-1 $\beta$ is only induced in response to inflammatory stimuli and its expression is not sustained during chronic treatment, while IL-18 is constitutively expressed and further induced after the stimulation in a type I IFN signaling-dependent manner.

Overall, this dissertation addresses protective roles for cytosolic nucleic acidsensing molecules, STING and AIM2, in colon cancer, uncovers an interplay between the AIM2 inflammasome and type IFN signaling during $F$. novicida infection, and demonstrates a novel function for GSDMD in regulating AIM2 inflammasome. The dissertation finally describes distinctive mechanisms governing inflammasome-associated cytokines IL-1 $\beta$ and IL-18. 


\section{TABLE OF CONTENTS}

CHAPTER 1. INTRODUCTION .....................................................................................1

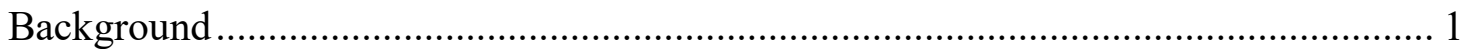

Cytosolic Nucleic Acids Sensing by Innate Immune Receptors................................. 1

Recognition of Cytosolic DNA by cGAS-STING Pathway and Their Potential

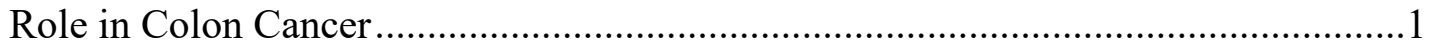

AIM2 in Colon Cancer and Francisella Infection ..................................................

Regulation of Inflammasome-Associated Cytokines IL-1 $\beta$ and IL-18 ......................5

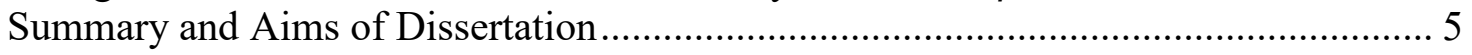

CHAPTER 2. MATERIALS AND METHODS.........................................................7

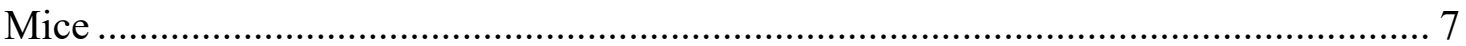

Bacterial Culture and Animal Infection ................................................................. 7

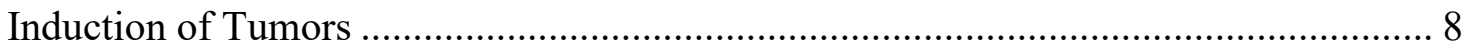

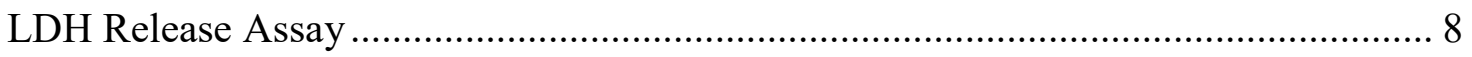

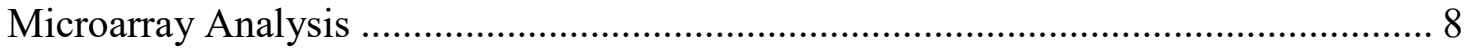

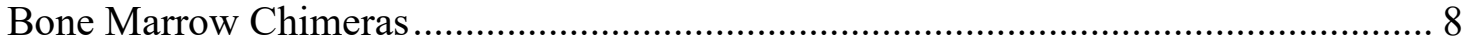

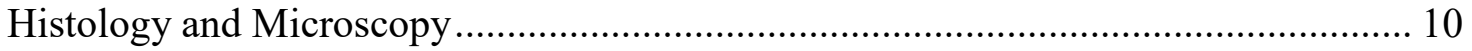

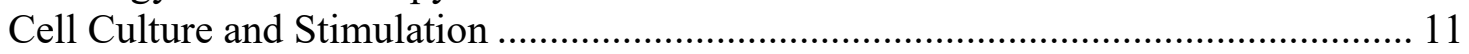

Cytokine Analysis ............................................................................................ 11

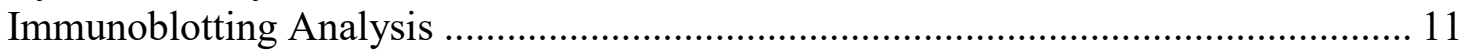

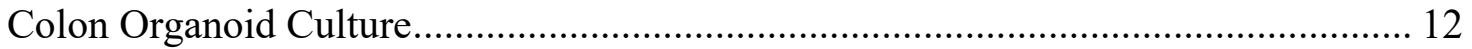

Real-time Quantitative PCR ............................................................................ 12

Universal Sequencing of Gut Microbiota ........................................................... 12

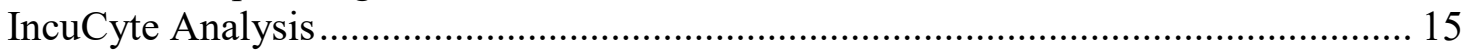

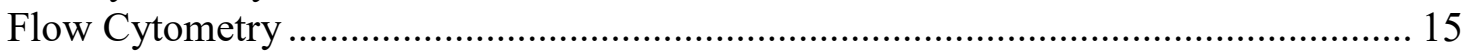

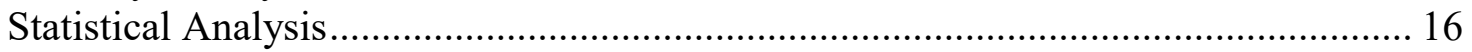

\section{CHAPTER 3. STING MEDIATES PROTECTION AGASINT COLON}

CANCER BY REGULATING COLON INFLAMMATION ......................................17

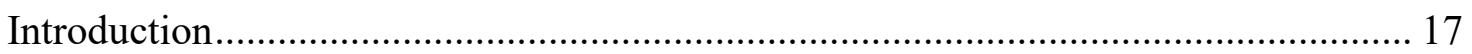

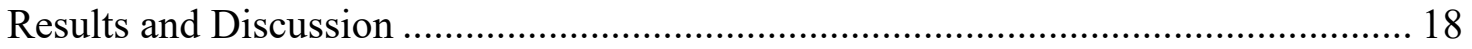

\section{CHAPTER 4. ROLE OF THE DNA SENSOR AIM2 IN COLON CANCER...........25}

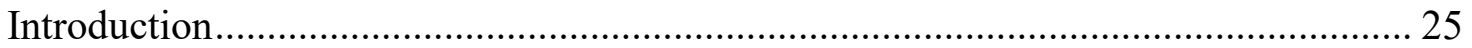

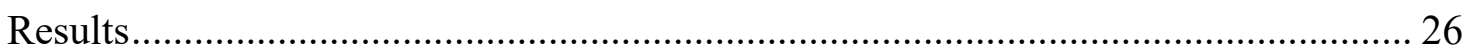

The DNA Receptor AIM2 Is Required to Prevent Colorectal Cancer......................26

AIM2 Controls Tumor Development Independently of Inflammasomes .................26

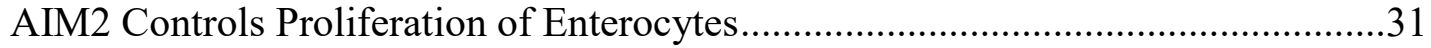

AIM2 Controls Expansion of Intestinal Stem Cells ................................................33

AIM2 Protects Colorectal Tumorigenesis by Modulating the Gut Microbiota .........40

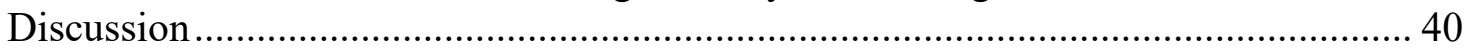




\section{CHAPTER 5. DETRIMENTAL TYPE I INTERFERON SIGNALING DOMINATES PROTECTIVE AIM2 INFLAMMASOME RESPONSES DURING FRANCISELLA NOVICIDA INFECTION}

Introduction.

Results and Discussion 45

IFNAR Signaling Enhances Susceptibility to Infection by $F$. novicida

The Detrimental Effects of Type I IFN Signaling Dominate the Protective Effects of AIM2 during $F$. novicida Infection in vivo

Inflammatory Cytokine Production Is Intact in the Absence of Type I IFN

Signaling

Type I IFN Signaling Controls Activation of Apoptotic Caspases and Cell Death...50

\section{CHAPTER 6. GASDERMIN D MEDIATES PROTECTION AGAINST \\ FRANCISELLA NOVICIDA BY PROMOTING INFLAMMASOME}

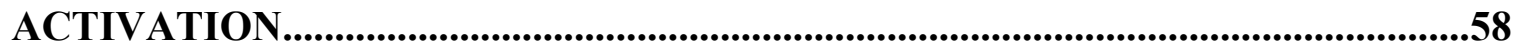

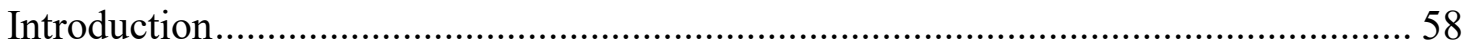

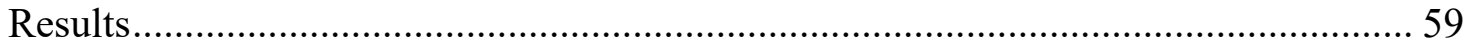

GSDMD Is Required for Host Defense Against $F$. novicida Infection ...................59

GSDMD Is Necessary for Optimal Caspase-1 Activation by F. novicida .................59

GSDMD Is Necessary for Optimal Caspase-1 Activation by Other AIM2

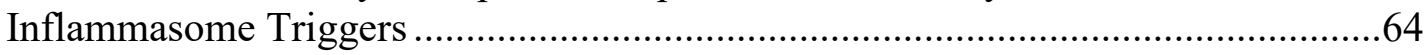

AIM2 Inflammasome Cleaves GSDMD during $F$. novicida Infection.....................64

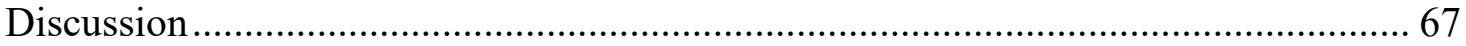

\section{CHAPTER 7. DISTINCT REGULATORY MECHANISMS CONTROL}

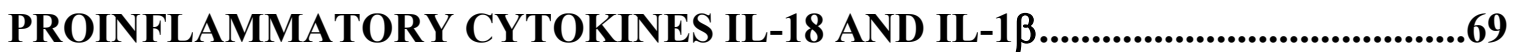

Introduction.

Results and Discussion

IL-18 and IL-1 $\beta$ Are Differentially Induced in Response to Inflammatory Stimuli..70 Induction of IL-18 but Not IL-1 $\beta$ Is Dependent on Type I IFN Signaling ................ 72 Components of Type I IFN Signaling Are Essential to Induce IL-18 ......................74

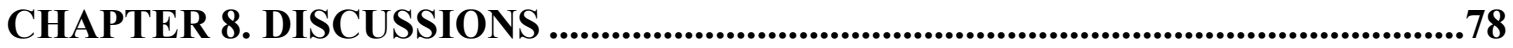

LIST OF REFERENCES ....................................................................................................81

VITA .93 


\section{LIST OF TABLES}

Table 2-1. Real-time qPCR primer sequences. .......................................................13 


\section{LIST OF FIGURES}

Figure 1-1. Diagram of cGAS-STING pathway.........................................................2

Figure 1-2. Diagram of AIM2 inflammasome in response to $F$. novicida . .....................4

Figure 2-1. Timeline for AOM and DSS treatment..............................................

Figure 3-1. STING is critical for mediating protection against colon tumor

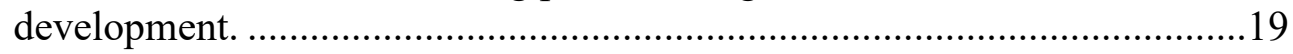

Figure 3-2. STING dampens early intestinal damage and overt proliferation. ..............20

Figure 3-3. STING suppresses overt colon inflammation during tumor development. ..22

Figure 3-4. The type I IFNs, caspase-1 and gut microbiota profile of WT and Sting ${ }^{g t / g t}$ mice.

Figure 4-1. AIM2 prevents colitis-associated colorectal tumorigenesis.

Figure 4-2. AIM2 governs colorectal tumorigenic susceptibility independently of inflammasomes and a number of inflammatory mediators.

Figure 4-3. AIM2 governs colorectal tumorigenic susceptibility independently of the magnitude of inflammation. ............................................................. 30

Figure 4-4. AIM2 suppresses overt proliferation. ................................................... 32

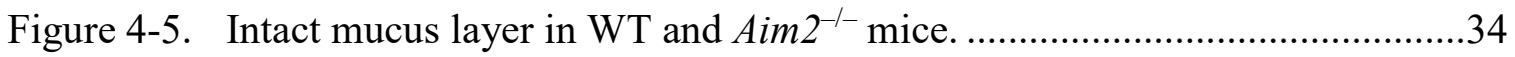

Figure 4-6. AIM2 controls proliferation of intestinal progenitor stem cells. .................35

Figure 4-7. Loss of AIM2 increased proliferation initiated by Prom $1^{+}$cells in the large intestine following $\beta$-catenin activation.

Figure 4-8. AIM2 controls expansion of intestinal stem cells...................................38

Figure 4-9. The role of hematopoietic and non-hematopoietic compartments in AIM2-mediated protection of colorectal tumorigenesis.

Figure 4-10. Reciprocal exchange of gut microbiota reduces susceptibility of Aim2 mice to colon cancer.

Figure 5-1. Mice lacking IFNAR1 or IFNAR2 are resistant to $F$. novicida. . .46

Figure 5-2. Detrimental role of type I IFN signaling is dominant over AIM2 inflammasome responses during $F$. novicida infection. . 
Figure 5-3. Characterization of Ifnar $2^{-/-} \mathrm{Aim}^{-/-}$mice.

Figure 5-4. Type I IFN signaling does not alter production of a range of inflammatory cytokines during $F$. novicida infection.

Figure 5-5. Inflammatory cytokine production after $F$. novicida infection.

Figure 5-6. Type I IFN signaling induces apoptotic cell death.

Figure 5-7. Analysis of apoptotic cell death and TRAIL pathway during F. novicida infection.

Figure 5-8. Opposing roles for type I IFNs signaling and AIM2 inflammasome responses during Francisella novicida infection in vivo..........................56

Figure 6-1. GSDMD contributes to host defense against $F$. novicida infection. 60

Figure 6-2. GSDMD regulates caspase-1 activation and cell death after various doses of $F$. novicida infection.

Figure 6-3. GSDMD promotes casaspse-1 activation by F. novicida.

Figure 6-4. TNF release is normal in the absence of GSDMD.

Figure 6-5. GSDMD is necessary for optimal casaspse-1 activation by other AIM2 inflammasome triggers.

Figure 6-6. GSDMD cleavage is dependent on AIM2 inflammasome. .66

Figure 7-1. IL-18 and IL-1 $\beta$ are differentially expressed in response to inflammatory stimuli.

Figure 7-2. Kinetics of IL-18 and IL-1 $\beta$ expression stimulated by gardiquimod.

Figure 7-3. Type I IFN signaling is essential for induction of IL-18 but not IL-1 $\beta \ldots . . . .73$

Figure 7-4. Components of type I IFN signaling are required for induction of IL-18 ...75

Figure 7-5. Type I IFNs induce IL-18 expression via ISGF3 and a model of differential regulation of pro-inflammatory cytokines IL-18 and IL-1 $\beta \ldots . . . .76$ 


\section{LIST OF ABBREVIATIONS}

$\begin{array}{ll}\text { AIM2 } & \text { Absent in melanoma 2 } \\ \text { AOM } & \text { Azoxymethane } \\ \text { BMDM } & \text { Bone marrow-derived macrophages } \\ \text { c-di-AMP } & \text { cyclic-di-AMP } \\ \text { c-di-GMP } & \text { cyclic-di-GMP } \\ \text { cGAMP } & \text { 2'3'-cyclic GMP-AMP } \\ \text { cGAS } & \text { cyclic GMP-AMP synthase } \\ \text { DAMPs } & \text { Danger-associated molecular patterns } \\ \text { DNA-PK } & \text { DNA-dependent protein kinase } \\ \text { dsDNA } & \text { Double-stranded DNA } \\ \text { DSS } & \text { Dextran sulfate sodium } \\ \text { E. coli } & \text { Escherichia coli } \\ \text { F.novicida } & \text { Francisella tularensis subspecies } \text { novicida } \\ \text { GBP } & \text { Guanylate-binding proteins } \\ \text { GSDMD } & \text { Gasdermin D } \\ \text { HMGB1 } & \text { High-mobility group box 1 } \\ \text { HSV } & \text { Herpes simplex virus } \\ \text { IFN } & \text { Interferon } \\ \text { IGF-1 } & \text { Insulin-like growth factor-1 } \\ \text { ISGF3 } & \text { Interferon-stimulated gene factor 3 } \\ \text { ISGs } & \text { Interferon-stimulated genes } \\ \text { IRF } & \text { Interferon-regulatory factor } \\ \text { IRGB10 } & \text { Immunity-related GTPase family member B10 } \\ \text { L. monocytogenes } & \text { Listeria monocytogenes } \\ \text { LRR } & \text { Leucine-rich repeat } \\ \text { MCMV } & \text { mouse cytomegalovirus } \\ \text { MDA5 } & \text { Melanoma differentiation-associated protein 5 } \\ \text { MIB } & \text { Mouse intestinal Bacteroides } \\ \text { MSH2 } & \text { MutS homolog 2 } \\ \text { NK } & \text { Natural killer } \\ \text { NLRP3 } & \text { Nucleotide-binding domain, leucine-rich repeat containing protein 3 } \\ \text { NOD } & \text { Nucleotidebinding oligomerization domain } \\ \text { PAMPs } & \text { Pathogen-associated molecular patterns } \\ \text { PCA } & \text { Principal Component Analysis } \\ \text { qPCR } & \text { Quantitative PCR } \\ \text { RIG-I } & \text { Retinoic acid inducible gene I } \\ \text { rTRAIL } & \text { Recombinant TRAIL } \\ \text { SFB } & \text { Segmented filamentous bacteria } \\ \text { STAT } & \text { Signal transducer and activator of transcription } \\ \text { STING } & \text { Stimulator of IFN genes } \\ \text { TBK1 } & \text { TANK-binding kinase 1 } \\ \text { TLR } & \text { Toll-like receptor } \\ \text { TRAIL } & \text { TNF-related apoptosis-inducing ligand } \\ & \end{array}$


TSB

WT

Trypticase Soy Broth

Wide-type 


\section{CHAPTER 1. INTRODUCTION}

\section{Background}

\section{Cytosolic Nucleic Acids Sensing by Innate Immune Receptors}

Pattern recognition receptors are germ-line encoded, innate immune sensors that recognize conserved molecules derived from microbes or disturbance of homeostatic selfcells, namely PAMPs or DAMPs. Cytosolic nucleic acids such as DNA and RNA form a major group of PAMPs and DAMPs, which can be utilized by microbes including viruses, bacteria and fungi as genetic materials and are sensed by cytosolic nucleic acids sensors upon being released into cytoplasm during the life cycle of the microbes. Under certain conditions, nucleic acids of self-origin gain access to the cytoplasm and are recognized by cytosolic sensors leading to autoimmunity [1].

RNA sensors retinoic acid inducible gene I (RIG-I) and melanoma differentiationassociated protein 5 (MDA5) recognize short and long RNA fragments respectively [2]. RIG-I also detects 5'-tri-phosphate and di-phosphate RNA [3-5]. A common adaptor, mitochondrial antiviral-signaling protein (MAVS) is activated downstream of RIG-I or MDA5 to induce production of type I IFNs and other inflammatory cytokines [6-9]. RIGI and MDA5 are critical in mediating immune responses to a variety of RNA viruses, such as influenza virus, hepatitis $C$ virus and myocarditis virus $[10,11]$. On the other hand, cytosolic DNA is sensed mainly by cyclic GMP-AMP synthase (cGAS)- STING pathway and AIM2, which will be discussed in details below.

\section{Recognition of Cytosolic DNA by cGAS-STING Pathway and Their Potential Role in Colon Cancer}

DNA sensor, cGAS, recognizes dsDNA in the cytoplasm and mediates synthesis of 2'3'-cyclic GMP-AMP (cGAMP), which binds and activates downstream adaptor

STING (Figure 1-1) [12-16]. Bacteria-derived cyclic-di-AMP (c-di-AMP) and cyclic-diGMP (c-di-GMP) in the cytoplasm can also bind and activate STING, placing STING as a direct sensor for cytosolic cyclic dinucleotides [17]. Activated STING recruits TANKbinding kinase 1 (TBK1) to induce phosphorylation of interferon-regulatory factor (IRF) 3 and IRF3-mediated expression of type I IFNs and other cytokines [18-20]. The adaptor STING has been shown to mount host defense against infection by DNA and RNA viruses and bacteria such as Francisella, Listeria and Mycobacterium genera [21]. The RNA-sensing pathway has been implicated to play a role in cancer biology in addition to its critical function in host defense against viral and bacterial infection. Treatment of DNA demethylation agents to colon tumor cells induces viral mimicry, leading to MDA5 and MAVS-dependent production of IFNs with anti-tumor abilities [23]. However, the role of the STING-mediated DNA-sensing pathway in cancer, particularly colon cancer, is rather understudied. Colon cancer is the 3rd most commonly diagnosed cancer and 4th 


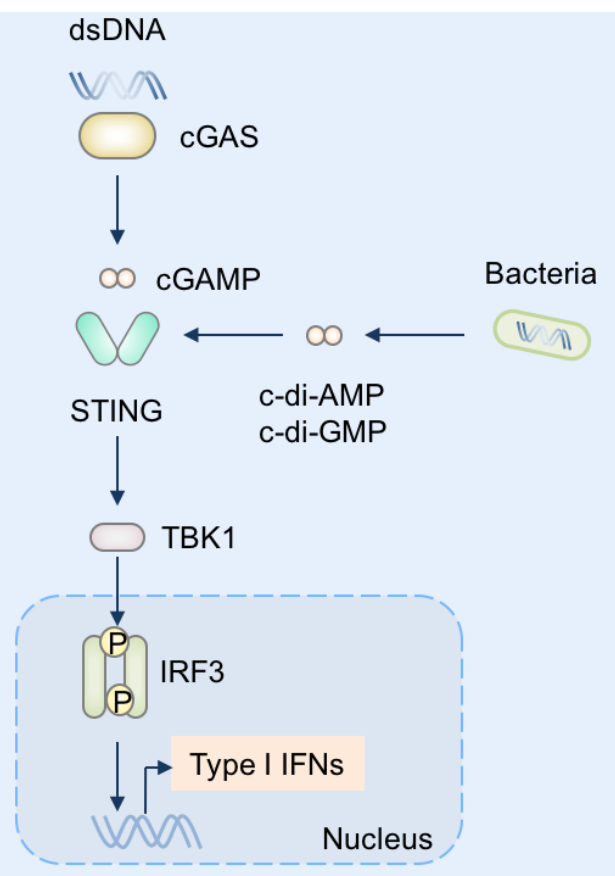

Figure 1-1. Diagram of cGAS-STING pathway.

cGAS senses and binds to cytosolic dsDNA leading to production of cGAMP. cGAMP then binds and activates the downstream adaptor STING to induce recruitment of TBK1 and phosphorylation of IRF3. Phosphorylated IRF3 forms dimers to enter the nucleus to mediate transcription of type I IFNs and other inflammatory cytokines. Bacteria such as L. monocytogenes release c-di-AMP and c-di-GMP into the cytosol and activate STING directly $[17,22]$. 
leading cause for cancer-related deaths worldwide [24]. The incidence and mortality of colon cancer are rapidly increasing in developing countries [25]. Inflammation functions as a driving force to promote colon tumorigenesis. Clinical data support this notion by observing increased probability for patients with ulcerative colitis to develop colon cancer [26]. Moreover, previous studies have suggested that innate immune sensors can modulate the magnitude of colon inflammation thereby regulating development of colon cancer [27-31], underscoring a potential role for the DNA-sensing pathway governed by cGAS and STING in colitis-associated cancer.

\section{AIM2 in Colon Cancer and Francisella Infection}

dsDNA in the cytoplasm can also be recognized by AIM2 (Figure 1-2) [32-35]. AIM2 binds to DNA via a HIN200 domain which allows its PYRIN domain to interact with the PYRIN domain of the bipartite adaptor molecule ASC [36]. The CARD domain of ASC recruits and interacts with the CARD domain of caspase-1 to form a multiprotein complex named the inflammasome [36]. The AIM2 inflammasome mediates activation of caspase-1 leading to caspase-1-depdendent maturation and secretion of IL$1 \beta$ and IL-18 and induction of pyroptosis. The AIM2 gene was initially isolated by investigating tumorigenicity of melanoma cells [37]. Later studies identified mutations and reduced expression of AIM2 gene in human colon tumors and colon tumor cell lines [38-40]. Moreover, a poorer survival rate has been reported in colon cancer patients bearing lower AIM2 expression in the tumors [40]. Besides its potential role in colon cancer, AIM2 has been established as a critical sensor for microbial infection. AIM2 is responsible for inducing inflammasome responses upon infection by viruses including mouse cytomegalovirus (MCMV) and vaccinia virus, bacteria including Francisella tularensis Live Vaccine Strain, F. novicida, Listeria monocytogenes (L. monocytogenes) and Mycobacterium, and fungi including Aspergillus fumigatus [1]. Of these, F. novicida is a gram-negative bacterium that exclusively activates AIM2 inflammasome in a type I IFN signaling-dependent manner in murine macrophages and dendritic cells (Figure 1-2) [41-43]. After being engulfed in macrophages, $F$. novicida escapes the Francisellacontaining vacuole and replicates in the cytoplasm. During this process, the cGASSTING pathway senses $F$. novicida leading to production of type I IFNs [44-46]. Type I IFNs induces expression of transcriptional factor IRF1 and further IRF1-dependent expression of immunity-related GTPase family member B10 (IRGB10) and guanylatebinding proteins (GBPs) including GBP2 and GBP5 [44, 45, 47]. IRGB10 and GBPs belong to a family of IFN-inducible GTPases and are proteins of cell-autonomous immunity [48]. During F. novicida infection, they are targeted to the cell membrane of bacteria leading to bacterial killing and release of bacterial ligands including DNA into the cytoplasm, thereby activating AIM2 inflammasome [44, 45, 47].

While AIM2 is required for host protection against $F$. novicida infection, the role of type I IFN signaling during bacterial infection in vivo is diverse, which can be either beneficial or detrimental depending on the pathogen. Type I IFNs provide protection to the mice infected with Escherichia coli (E. coli), Streptococcus pneumoniae, or Streptococcus pyogenes but are deleterious following infection by $F$. novicida, 


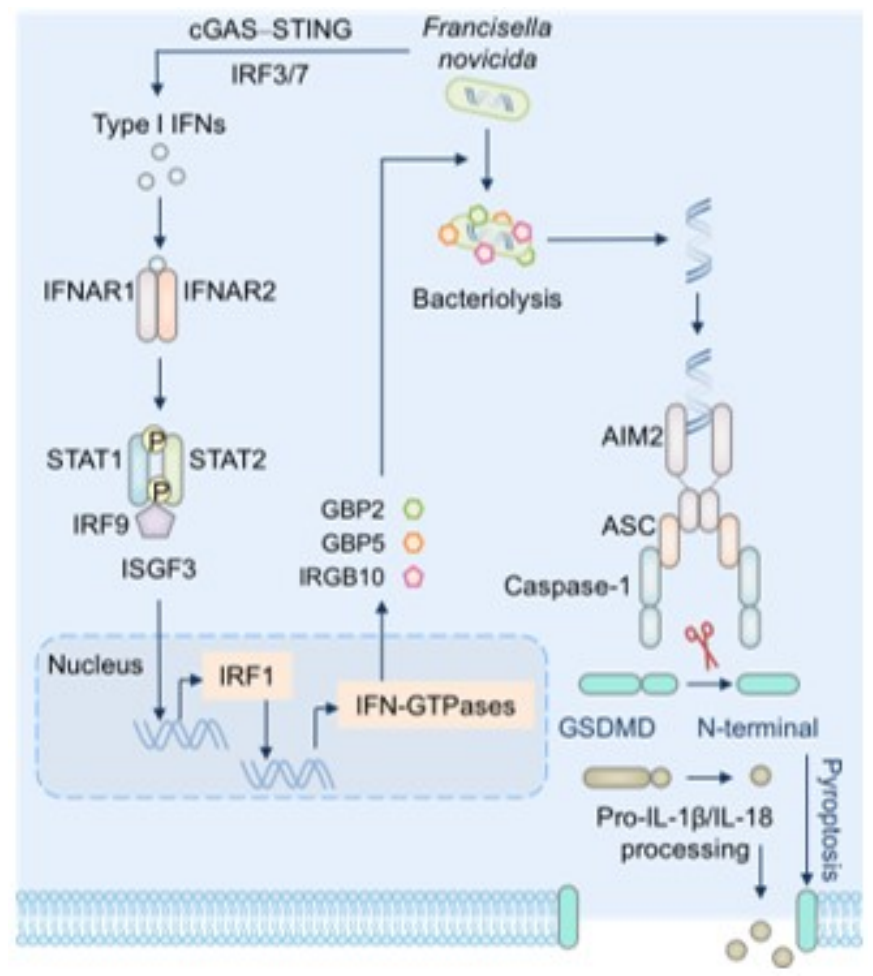

Figure 1-2. Diagram of AIM2 inflammasome in response to $F$. novicida.

Cytosolic $F$. novicida is recognized by the cGAS-STING pathway leading to production of type I IFNs via transcription factors IRF3 and IRF7. Type I IFNs bind to the heterodimeric receptor consisting of IFNAR1 and IFNAR2 to induce formation of a signaling transducer named interferon-stimulated gene factor 3 (ISGF3), which include signal transducer and activator of transcription (STAT) members STAT1, STAT2 and IRF9 to mediate expression of IRF1 and in turn IRF1-dependent expression of IRGB10 and GBPs. These IFN-inducible GTPases are recruited to the bacteria membrane to induce bacteriolysis and release of bacterial DNA into the cytosol to activate the AIM2 inflammasome. TheAIM2 inflammasome activates caspase-1. Activated caspase-1 cleaves GSDMD to release the $\mathrm{N}$-terminal portion to induce pyroptosis by generating pore structures on the cell plasma membrane. Caspase- 1 also cleaves pro-IL- $1 \beta$ and proIL-18 into their mature form. IL-1 $\beta$ and IL-18 are released from the cell through the pores formed by GSDMD. 
Salmonella enterica, or L. monocytogenes [49, 50]. How the interplay between the opposing effects of both type I IFN signaling and the AIM2 inflammasome determines the final outcome during host defense against $F$. novicida infection has remained unclear.

In addition, recent studies have discovered GSDMD as the molecular basis underlying pyroptosis downstream of inflammasome activation [51-53] (Figure 1-2). GSDMD belongs to gasdermin family of proteins. Inflammatory caspases including caspase-11 or caspase-1 cleave a linker region between the $\mathrm{N}$ - and $\mathrm{C}$-terminal domain of GSDMD, releasing the functional $\mathrm{N}$-terminal fragment from inhibition by the $\mathrm{C}$-terminal end [51-53]. The N-terminal portion of GSDMD has been shown to be associated with lipid membranes with an ability to directly bind to cardiolipin and phosphoinositides, the latter of which are usually located in the inner leaflet of the plasma membrane [54-58]. GSDMD forms pores on the membrane and induces cell lysis. In addition, GSDMDformed pores allow release of IL-1 $\beta$ and IL-18 independently of pyroptosis $[59,60]$. While IL-1 $\beta$ and IL-18 have been shown to protect against Francisella tularensis Live Vaccine Strain infection downstream of AIM2 inflammasome activation [61, 62], the role of AIM2-mediated pyroptosis during Francisella infection has not been studied yet.

\section{Regulation of Inflammasome-Associated Cytokines IL-1 $\beta$ and IL-18}

IL- $1 \beta$ and IL-18 are members of the IL- 1 cytokine family and are synthesized as precursor proteins. Upon the assembly of inflammasome, active caspase-1 cleaves IL-1 $\beta$ and IL-18 leading to their subsequent release from the cell [63]. Interestingly, although IL-1 $\beta$ is not expressed under steady state, a cellular pool of IL-18 already exists before any inflammatory stimuli, and is ready to be activated and released by the inflammasome [64]. Expression of IL-1 $\beta$ is induced when cells are stimulated with different toll-like receptor (TLR) ligands or cytokines [65-67]. Several reports suggest that IL-18 expression can also be induced by several TLR ligands or sendai virus [68-71]. Thus, how the levels of IL-1 $\beta$ and IL-18 are differentially regulated by inflammatory signals is unclear.

\section{Summary and Aims of Dissertation}

Upon recognition of dsDNA in the cytoplasm, STING mediates expression of type I IFNs and other inflammatory genes, while AIM2 forms an inflammasome to induce IL-1 $\beta$ and IL-18 release and pyroptosis. Both DNA-sensing pathways are critical in infection and autoimmune diseases, while their roles in cancer are yet to be studied. First two aims of this dissertation is to investigate potential functions of STING and AIM2 in colon cancer. We hypothesize that they have critical roles during colon cancer development.

Upon $F$. novicida infection in vitro, AIM2 senses bacterial DNA derived from $F$. novicida to form the inflammasome, a process that is dependent on type I IFN signaling. However, while the AIM2 inflammasome is protective against $F$. novicida infection, type 
I IFN signaling is detrimental to the host during the infection in vivo. The third aim of the dissertation is to address the interplay between AIM2 inflammasome responses and type I IFN signaling during $F$. novicida infection in vivo. Thus, we hypothesize that the detrimental type I IFN signaling could interference with the protective AIM2 inflammasome responses.

The AIM2 inflammasome activates caspase-1 to induce GSDMD-mediated pyroptosis and release of IL-1 $\beta$ and IL-18. Fourth aim of the dissertation is to study the role of GSDMD downstream of the AIM2 inflammasome during F. novicida infection. We hypothesize that GSDMD functions to provide protection to the host during $F$. novicida infection.

The fifth aim of the dissertation is to characterize mechanisms governing expression of IL-1 $\beta$ and IL-18 in response to different inflammatory stimuli. 


\section{CHAPTER 2. MATERIALS AND METHODS*}

\section{Mice}

Wide-type (WT) C57BL/6J mice and $\operatorname{Irf} 8^{\text {tm } 1.2 H m} / \mathrm{J}$ mice (also known as $\operatorname{Irf} 8^{-/-}$; Stock Number 018298) were purchased from The Jackson Laboratory. STING-deficient

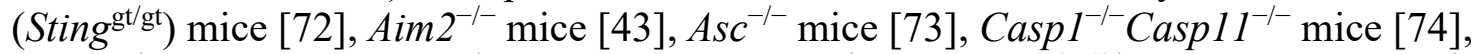
Casp3 $^{-/-}$mice [75], Casp $7^{-/-}$mice [76], Prom ${ }^{\mathrm{C}-\mathrm{L} /+} ;$ Ctnnbllox $^{(\mathrm{ex} 3) /+}$ mice [77], Ifnarl ${ }^{-1-}$ mice [78], Ifnar2 ${ }^{-/-}$mice [79], Irf3 ${ }^{-/-}$mice [80], Irf4 $4^{\mathrm{fl} / \mathrm{fl}}-\mathrm{Lysm}$-Cre mice [81], Irf5 ${ }^{-/-}$mice

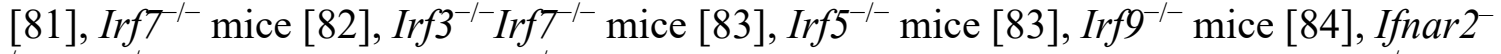
${ }^{1-}$ Aim $^{-1-}$ mice [83] and Gsdmd ${ }^{-/-}$mice [85] have been described previously. Stat $1^{-/-}$mice were provided by Dr. Abhay Satoskar (Ohio State University). Male and female mice of 6-8 weeks old were used in this study. All mice were bred at the Animal Resource Center at St. Jude. Animal studies were conducted according to protocols approved by the St. Jude Animal Care and Use Committee.

\section{Bacterial Culture and Animal Infection}

F. novicida strain U112 was grown overnight at $37^{\circ} \mathrm{C}$ in BBL Trypticase Soy Broth (TSB) (BD) contaning 0.2\% L-cysteine and were 1:10 subcultured for 4 hours. Mice were infected subcutaneously with $F$. novicida in $200 \mu \mathrm{l}$ PBS. For CFU analysis, homogenized liver and spleen tissue samples were plated onto TSB agar containing $0.2 \%$

\footnotetext{
* Adapted with permission. Zhu Q., Man S.M., Gurung P., Liu Z., Vogel P., Lamkanfi M., Kanneganti T.D. (2014). Cutting Edge: STING mediates protection against colorectal tumorigenesis by governing the magnitude of intestinal inflammation. Journal of Immunology. 193(10):4779-82. Copyright 2014. The American Association of Immunologists, Inc.
}

Adapted with open access permission. Man S.M.*, Zhu Q.*, Zhu L.*, Liu Z.*, Karki R., Malik A., Sharma D., Li L., Malireddi R.K., Gurung P., Neale G., Olsen S.R., Carter R.A., McGoldrick D.J., Wu G., Finkelstein D., Vogel P., Gilbertson R.J., Kanneganti T.D. (2015). Critical role for the DNA sensor AIM2 in stem cell proliferation and cancer. Cell. 162(1):45-58. (* Co-first author)

Adapted with permission. Zhu, Q. and T.D. Kanneganti, Cutting Edge: Distinct Regulatory Mechanisms Control Proinflammatory Cytokines IL-18 and IL-1 $\beta$. J Immunol, 2017. 198(11): p. 4210-4215. Copyright 2017. The American Association of Immunologists, Inc.

Adapted with open access permission. Zhu, Q., Man S.M., Karki R., Malireddi R.K., Kanneganti, T.D. (2018). Detrimental type I Interferon signaling dominates protective AIM2 Inflammasome responses during Francisella novicida Infection. Cell Reports. 22, 3168-3174. 
L-cysteine and incubated overnight. For survival analysis following TNF-related apoptosis-inducing ligand (TRAIL) neutralization, mice were injected intraperitoneally with $300 \mathrm{mg}$ anti-TRAIL monoclonal antibody (N2B2) or immunoglobulin G2a (IgG2a) (RTK2758) isotype control (BioLegend) on days 0,1 , and 2 post-infection. For the recombinant TRAIL (rTRAIL) experiment, mice were injected intraperitoneally with 10 mg rTRAIL (PeproTech) or PBS on the same day as infection.

\section{Induction of Tumors}

For azoxymethane (AOM) and dextran sulfate sodium (DSS) model (Figure 2-1) [86], mice were injected intraperitoneally with $10 \mathrm{mg}$ of AOM (Sigma) per kg body weight. 5 days later, 3\% DSS (molecular mass 36-40 kDa; MP Biologicals) was given in the drinking water for 6 days, which was further followed by drinking water for 2 weeks. This cycle was repeated twice with $2.5 \%$ DSS. Mice were sacrificed on day 80 . For Day 8 samples, mice were injected with AOM, fed 3\% DSS for 3 days and sacrificed. For Day 14 samples, mice were injected with AOM, fed 3\% DSS for 6 days and sacrificed 3 days later. For co-housing experiments, equal numbers of female WT and female $\mathrm{Aim}^{-/-}$mice were housed in the same cage for 4 weeks. Mice were then housed separately before AOM injection and remained separated over the course of the experiment. To induce oncogenic Wnt signaling, tamoxifen induction was performed on 2-month-old mice by oral gavage with $200 \mathrm{ml}$ tamoxifen $(20 \mathrm{mg} / \mathrm{ml})$ in corn oil for 2 days.

\section{LDH Release Assay}

Cell culture supernatants were collected at the indicated times, and LDH activity was measured using the Promega cytotoxicity kit according to manufacturer's protocols.

\section{Microarray Analysis}

Mouse Gene 2.0 (Affymetrix) was used to examine the gene expression profiles using RNA that was extracted from colon tissues. GSEA software (Broad Institute) was used to analyze the relevant signaling pathways.

\section{Bone Marrow Chimeras}

Mice were subjected to split-dose irradiation, first with a 350 rad dose, followed by a 950 rad dose 24 hours later. 2 hours after the second dose of irradiation, mice were injected intravenously with $10^{7}$ bone marrow cells from the femur and tibia of donor mice. The following four groups of chimeric mice were generated: WT $\rightarrow$ WT (CD45.1expressing WT cells into CD45.2-expressing WT mice), Aim $2^{--} \rightarrow$ WT (CD45.2expressing Aim $2^{-/-}$cells into CD45.1-expressing WT mice), WT $\rightarrow$ Aim2 $2^{-/}$(CD45.1expressing WT cells into CD45.2- expressing Aim $2^{-/-}$mice) and Aim2 $2^{-/-} \rightarrow \mathrm{Aim}^{\mathrm{C}^{--}}$ 


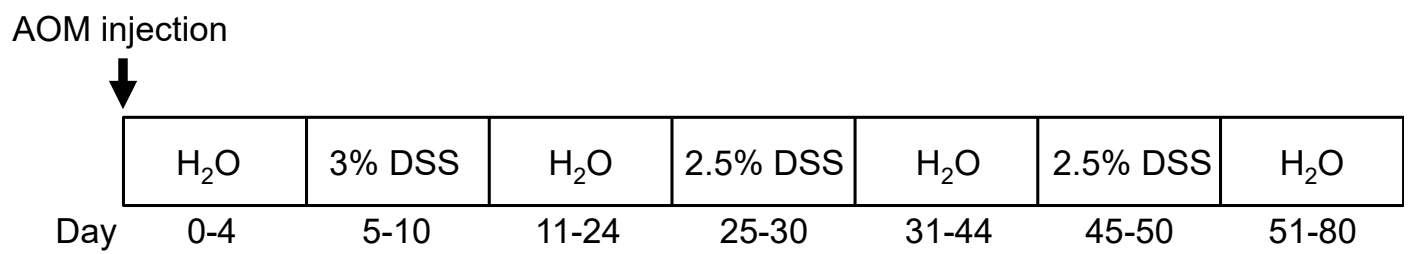

Figure 2-1. Timeline for AOM and DSS treatment. 
(CD45.2-expressing Aim2 $2^{-/-}$cells into CD45.2-expressing $\mathrm{Aim} 2^{-/-}$mice). The reconstitution rate evaluated 6 weeks after bone marrow transfer was determined by flow staining of CD45.1 and CD45.2 in blood leukocytes, which was at least 95\%.

\section{Histology and Microscopy}

Tissues were fixed in 10\% formalin, embedded in paraffin and sectioned. Colon tissues were stained with H\&E. The number of proliferating cells in the intestinal epithelium was detected by immunoperoxidase staining for thymidine analog 5'-bromo2 'deoxyuridine (BrdU). In brief, $1 \mathrm{mg} / \mathrm{ml} \mathrm{BrdU}$ in PBS was injected intraperitoneally. Colon tissues were collected after 2 hours and fixed in 10\% neutral buffered formalin and embedded in paraffin. Immunohistochemistry was performed using the in situ BrdU staining kit according to manufacturer's instructions (BD Bioscience, 550803). Ki67 (NBP1-40684, Novus Biologicals), AKT (4691, Cell Signaling Technology), p-AKT (Ser473; 4060, Cell Signaling Technology), and c-Myc (5605, Cell Signaling Technology) staining were performed using antibodies according to the manufacturers' instructions. The number of Ki67- or BrdU-positive cells per crypt in each animal was counted (at least 18-20 crypts per mouse) blindly. Tissues were counterstained with hematoxylin. For staining of goblet cells, colon tissues were stained with PAS and Alcian blue. The number of goblet cells per crypt was counted blindly. For nLacZ analysis and GFP microscopy, intestinal tissues were perfused and fixed in $2 \%$ paraformaldehyde overnight, cryo-protected, and frozen in OCT. Sections were stained for $\beta$-galactosidase or examined for GFP signal. For $\beta$-galactosidase staining, frozen sections were washed in PBS for $5 \mathrm{~min}$ and incubated in the dark in standard $\beta$-galactosidase substrate $(5 \mathrm{mM}$ potassium ferricyanide, $5 \mathrm{mM}$ potassium ferrocyanide, $1 \mathrm{mg} / \mathrm{ml} \mathrm{X-gal,} 2 \mathrm{mM} \mathrm{MgCl}_{2}$, $0.01 \%$ sodium deoxycholate, and $0.02 \% \mathrm{NP}-40$ in PBS) for 4 hours at $37^{\circ} \mathrm{C}$. Sections were then rinsed and counterstained by Nuclear Fast Red (Vector, H-3403). For TUNEL (terminal deoxynucleotidyl transferase deoxyuridine triphosphate nick end labeling) staining of liver tissues after $F$. novicida infection, the staining was performed using In Situ Cell Death Detection Kit (11684817910) according to the manufacturer's instructions (Sigma).

Histological lesions were scored blindly by a pathologist for following the categories: inflammation severity, ulceration, hyperplasia and lesion area. Inflammation severity was assigned as follows: $0=$ normal; $1=$ mild (small, focal, or widely separated, mostly limited to lamina propria); 2 = moderate (multifocal mucosal inflammation, often extending to submucosa); 3 = marked (multifocal and coalescing to extensive inflammation with occasional transmural foci); $4=$ severe (diffuse inflammation with transmural inflammation). Scores for ulceration were assigned as follows: $0=$ normal (no ulcers); $1=$ mild ( $1-2$ ulcers, involving up to a total of 20 crypts); $2=$ moderate (1-4 ulcers, involving a total of 20-40 crypts); $3=$ severe (more than 4 ulcers or over 40 crypts). Hyperplasia scores were assigned as follows: $0=$ normal; $1=$ mild (Crypts elongated, normal epithelium); 2 = moderate (Crypts elongated with crowding of hyperchromatic epithelium, no branching of crypts); 3 = marked (Crypts 2-3 times normal thickness, hyperchromatic epithelium, reduced goblet cells, scattered 
arborization); 4 = severe (Crypts 2-3 times normal thickness, marked hyperchromasia, few to no goblet cells, high mitotic index, frequent arborization). Scoring for lesion areas were assigned as follows: $0=$ normal $(0 \%$ involvement); $1=$ minimal (less than $10 \%$ involvement); $2=$ mild $(10 \%$ to $25 \%$ involvement $) ; 3=$ moderate $(25 \%$ to $50 \%$ involvement); $4=$ marked ( $50 \%$ to $75 \%$ involvement); $5=$ severe (over $75 \%$ involvement).

\section{Cell Culture and Stimulation}

Bone marrow-derived macrophages (BMDMs) were cultured for 6 days in DMEM containing 10\% FBS, 30\% L929 conditioned media and 1\% penicillin and streptomycin, and seeded at a concentration of $1 \times 10^{6}$ cells onto 12 -well plates. The next day, BMDMs were incubated in antibiotic-free media. BMDMs were stimulated with LPS (500 ng/mL; InvivoGen), Pam3CSK4 $(1 \mu \mathrm{g} / \mathrm{mL})$, gardiquimod $(1 \mu \mathrm{g} / \mathrm{mL}$; InvivoGen), Poly(I:C) (10 $\mu \mathrm{g} / \mathrm{mL}$; InvivoGen), or IFN $\beta$ (400 U/mL; PBL Assay Science). For transfection of poly(dA:dT), $0.25 \mu \mathrm{g}$ or 1 ug of poly(dA:dT) (InvivoGen) was mixed with $0.3 \mathrm{~mL}$ of Xfect polymer in Xfect reaction buffer (Clontech Laboratories) for 10 min and then added to BMDMs in Opti-MEM (ThermoFisher Scientific). For transfection of cGAMP, $1 \mu \mathrm{g}$ of cGAMP (InvivoGen) was mixed with $0.3 \mathrm{~mL}$ of Xfect polymer in Xfect reaction buffer (Clontech Laboratories) for 10 min and then added to BMDMs in Opti-MEM (ThermoFisher Scientific). F. novicida was cultured and added to unprimed BMDMs at an indicated MOI for 20 hours. $50 \mu \mathrm{g} / \mathrm{ml}$ gentamicin was added after 4 hours of the infection. The MCMV Smith MSGV strain (VR-1399, American Type Culture Collection) was obtained from P.G. Thomas (St. Jude) and was used in experiments in Chapter 5. The MCMV strain (K181) was obtained from Edward S. Mocarski (Emory University School of Medicine) [87] and was used in experiments in Chapter 6. MCMV was added at an MOI of 10 for 10 hours.

\section{Cytokine Analysis}

Levels of cytokines were measured by ELISA according to the manufacturer's instructions (IL-18 ELISA from eBioscience, IFN- $\beta$ from BioLegend and all other cytokines were from Millipore).

\section{Immunoblotting Analysis}

Proteins from tissues were extracted using RIPA buffer supplemented with protease and phosphatase inhibitors (Roche). BMDMs samples were prepared as previously described [81, 83]. Prepared samples were then separated by SDS-PAGE and transferred to polyvinylidene difluoride membranes (Millipore). Membranes were blocked by $5 \%$ milk and incubated with primary antibodies overnight. The primary antibodies used were, caspase-1 p10 (sc-515, Santa Cruz), p-ERK (9101, Cell Signaling Technology), ERK (9102, Cell Signaling Technology), p-IкBa (9241, Cell Signaling 
Technology), IкBa (9242, Cell Signaling Technology), STAT3 (9139, Cell Signaling Technology), p-STAT3 (9131, Cell Signaling Technology), HIF-1 $\alpha$ (3716, Cell Signaling Technology), p-AKT Ser473 (9271, Cell Signaling Technology), AKT (4691, Cell Signaling Technology), p-PTEN (9554, Cell Signaling Technology), PTEN (9552, Cell Signaling Technology), c-Myc (5605, Cell Signaling Technology), caspase-1 (AG20B-0042, Adipogen), caspase-8 (ALX-804-447-C100, Enzo Life Sciences), cleaved caspase-8 (8592, Cell Signaling Technology), caspase-3 (9662, Cell Signaling Technology), cleaved caspase-3 (9661, Cell Signaling Technology), caspase-7 (9492, Cell Signaling Technology), cleaved caspase-7 (9491, Cell Signaling Technology), TRAIL (AF1121, R\&D Systems), DR5 (MAB1540, R\&D Systems), GAPDH (5174, Cell Signaling Technology), GSDMD (ab209845, Abcam), IL-18 (D046-3, MBL International), IL-1 $\beta$ (AF-401-NA, R\&D Systems), and $\beta$-actin (8457, Cell Signaling Technology). Membranes were then incubated with HRP-conjugated secondary antibody for 1 hour and proteins were visualized using ECL substrate (ThermoScientific) or Luminata Forte Western HRP substrate (Millipore). Immunoblots were quantified using ImageJ in Chapter 5.

\section{Colon Organoid Culture}

Mouse colon stem cells were cultured as previously described with modifications [88]. Briefly, mice were perfused with prewarmed $30 \mathrm{mM}$ EDTA in PBS. The colon was removed and rinsed with PBS. Colonic mucosa was scraped from the muscle layer and kept in $10 \mathrm{mM}$ EDTA in PBS on ice and incubated in a shaker at 350 RPM for $15 \mathrm{~min}$ at $4{ }^{\circ} \mathrm{C}$. Dissociated colonic crypts were vigorously suspended. Cells were filtered through a $70 \mathrm{~mm}$ cell strainer, mixed with Matrigel (BD Bioscience, 354230) and plated in $60 \mathrm{~mm}$ plates. The culture medium consisted of Advanced DMEM/F12 supplemented with B27, $\mathrm{N} 2$, N-Acetylcysteine, Gastrin $(10 \mathrm{nM})$, and growth factors, including $50 \mathrm{ng} / \mathrm{ml} \mathrm{EGF,} 1$ $\mathrm{mg} / \mathrm{ml}$ R-spondin1, $100 \mathrm{ng} / \mathrm{ml}$ Noggin, $100 \mathrm{ng} / \mathrm{ml} \mathrm{FGF10,} \mathrm{and} \mathrm{Wnt3A} \mathrm{conditioned} \mathrm{media}$ [89].

\section{Real-time Quantitative PCR}

RNA was extracted using TRIzol reagent (Life Technologies). Isolated RNA was reverse transcribed into cDNA using the First-Strand cDNA Synthesis Kit (Life Technologies). Real-time quantitative PCR (qPCR) was performed on an ABI 7500 realtime PCR instrument with $2 \times$ SYBR Green (Applied Biosystems). Primer sequences in

Table 2-1.

\section{Universal Sequencing of Gut Microbiota}

Genomic DNA from feces was extracted with the QiAamp DNA stool Mini Kit (QIAGEN) and used for 16S rRNA gene sequencing. Libraries were prepared by amplification of the 16S v4 domain according to the manufacturer's instructions (Bioo 
Table 2-1. Real-time qPCR primer sequences.

\begin{tabular}{|c|c|}
\hline Target & $\begin{array}{c}\text { Primer sequence } \\
\end{array}$ \\
\hline \multirow[t]{2}{*}{ Sting } & Forward: 5'-CAT TGG GTA CTT GCG GTT-3' \\
\hline & Reverse: 5'-CTG AGC ATG TTG TTA TGT AGC-3' \\
\hline \multirow[t]{2}{*}{$\operatorname{Aim} 2$} & Forward: 5'-GAT TCA AAG TGC AGG TGC GG-3' \\
\hline & Reverse: 5'-TCT GAG GCT TAG CTT GAG GAC-3' \\
\hline \multirow[t]{2}{*}{ Gapdh } & Forward: 5'-CGT CCC GTA GAC AAA ATG GT-3' \\
\hline & Reverse: 5'-TTG ATG GCA ACA ATC TCC AC-3' \\
\hline \multirow[t]{2}{*}{$I l 17 a$} & Forward: 5'-TCC AGA AGG CCC TCA GAC TA-3' \\
\hline & Reverse: 5'-ACA CCC ACC AGC ATC TTC TC-3' \\
\hline \multirow[t]{2}{*}{ Il22 } & Forward: 5'-TCC GAG GAG TCA GTG CTA AA-3' \\
\hline & Reverse: 5'-AGA ACG TCT TCC AGG GTG AA-3' \\
\hline \multirow[t]{2}{*}{$1123 p 19$} & Forward: 5'-GGT GGC TCA GGG AAA TGT-3' \\
\hline & Reverse: 5'-GAC AGA GCA GGC AGG TAC AG-3' \\
\hline \multirow[t]{2}{*}{ Ifnb } & Forward: 5'-GCC TTT GCC ATC CAA GAG ATG C-3' \\
\hline & Reverse: 5'-ACA CTG TCT GCT GGT GGA GTT C-3' \\
\hline \multirow[t]{2}{*}{ Ifng } & Forward: 5'-GCA TCT TGG CTT TGC AGC T-3' \\
\hline & Reverse: 5'-CCT TTT TCG CCT TGC TGT TG-3' \\
\hline \multirow[t]{2}{*}{$\operatorname{Tg} f b 1$} & Forward: 5'-CTC CCG TGG CTT CTA GTG C-3' \\
\hline & Reverse: 5'-GCC TTA GTT TGG ACA GGA TCT G-3' \\
\hline \multirow[t]{2}{*}{$T g f b 2$} & Forward: 5'-TCG ACA TGG ATC AGT TTA TGC G-3' \\
\hline & Reverse: 5'-CCC TGG TAC TGT TGT AGA TGG A-3' \\
\hline \multirow[t]{2}{*}{ Illo } & Forward: 5'-GCT CTT ACT GAC TGG CAT GAG-3' \\
\hline & Reverse: 5’-CGC AGC TCT AGG AGC ATG TG-3' \\
\hline \multirow[t]{2}{*}{ Мuc2 } & Forward: 5'-GCT GAC GAG TGG TTG GTG AAT G-3' \\
\hline & Reverse: 5'-GAT GAG GTG GCA GAC AGG AGA C-3' \\
\hline \multirow[t]{2}{*}{ Muc3 } & Forward: 5'-CGT GGT CAA CTG CGA GAA TGG-3' \\
\hline & Reverse: 5'-CGG CTC TAT CTC TAC GCT CTC C-3' \\
\hline \multirow[t]{2}{*}{ Muc4 } & Forward: 5'-CAG CAG CCA GTG GGG ACA G-3' \\
\hline & Reverse: 5'-CTC AGA CAC AGC CAG GGA ACT C-3' \\
\hline \multirow[t]{2}{*}{$c M y c$} & Forward: 5'-TCT CCA CTC ACC AGC ACA ACT ACG-3' \\
\hline & Reverse: 5'-ATC TGC TTC AGG ACC CT-3' \\
\hline \multirow[t]{2}{*}{$5100 a 9$} & Forward: 5’-GGT GGA AGC ACA GTT GGC A-3' \\
\hline & Reverse: 5'-GTG TCC AGG TCC TCC ATG ATG-3' \\
\hline \multirow[t]{2}{*}{ Snrpdl } & Forward: 5'-CAG CAT GAA CAC ACA CCT TAA AG-3' \\
\hline & Reverse: 5’-GCC TCG AAT ACT CAA TGT TTC CA-3' \\
\hline \multirow[t]{2}{*}{$D b f 4$} & Forward: 5'-AAT AAG ATA CAG TGT CGG GTC CC-3' \\
\hline & Reverse: 5’- GTC CTT CTG GAA ATT GGG CTC-3' \\
\hline \multirow[t]{2}{*}{ Ill18 } & Forward: 5'-GCC TCA AAC CTT CCA AAT CA-3' \\
\hline & Reverse: 5’-TGG ATC CAT TTC CTC AAA GG-3' \\
\hline \multirow[t]{2}{*}{ Ill $1 \mathrm{~b}$} & Forward: 5'-GAC CTT CCA GGA TGA GGA CA-3' \\
\hline & Reverse: 5'-AGC TCA TAT GGG TCC GAC AG-3' \\
\hline \multirow[t]{2}{*}{ Trail } & Forward: 5'- ACC TCA GCT TCA GTC AGC ACT TC -3' \\
\hline & Reverse: 5'- TGT AAG TCA CAG CCA CAG ACA CAG -3' \\
\hline $\operatorname{Dr} 5$ & Forward: 5’- AGT GTG TCT CCA AAA CGG -3' \\
\hline
\end{tabular}


Table 2-1. Continued.

\begin{tabular}{ll}
\hline Target & \multicolumn{1}{c}{ Primer sequence } \\
\hline \multirow{3}{*}{-actin } & Reverse: 5'-AAT GCA CAG AGT TCG CAC T -3' \\
& Forward: 5'-CAG CTT CTT TGC AGC TCC TT-3', \\
Hprt & Reverse: 5'-CAC GAT GGA GGG GAA TAC AG-3' \\
& Forward: 5'-CTC ATG GAC TGA TTA TGG ACA GGA C-3' \\
& Reverse: 5'-GCA GGT CAG CAA AGA ACT TAT AGC C-3' \\
\hline
\end{tabular}


Scientific Corporation). Paired-end 150 cycle sequencing was performed on a MiSeq using 300 cycle v2 sequencing kits according the manufacturer's instructions (Illumina) with the following modifications: a phiX library was spiked in at $\sim 10 \%$, and library specific sequencing primers were added to the Illumina primer wells as specified by the manufacturer (Bioo Scientific Corporation). Primers targeting the 16S V4 region were aligned to the full set of sequences from the Greengenes database v13.5 [90] using exonerate [91]. Each sequence was truncated to include only the V4 region, the primermatching regions and an additional 40 bases on either side. Duplicate V4 regions were removed from the dataset. All taxa labels from the removed duplicates were associated with the remaining representative V4 region sequence. Reads from each sample were aligned exhaustively to the non-redundant V4 sequences using USEARCH [92] allowing a minimum sequence identity of $90 \%$. All taxon labels associated with the top-scoring V4 region(s) were used to determine the taxon assignment of each read. The highest resolution non-conflicting taxon from all taxa associated with the top-scoring V4 region was assigned as the taxa for a read. Relative proportions of microbial taxa for each sample were assembled from the highest resolution sequence counts into a matrix with samples as columns and taxa as rows with proportions in cells. Columns were also assigned to either a WT or $\mathrm{Aim}^{-/-}$group. All relative proportions were transformed to near normality with a shifted logit-p transformation [86]. Unpaired T-test with unequal variance for the normalized proportions and a two-factor ANOVA model was used to investigate significant taxa. Transformed values were then normalized for each taxa to produce a signal to noise ratio [86]. Signal to noise ratios are depicted in a heatmap plot and Principal Component Analysis (PCA) were generated with Spotfire (Tibco software Inc).

\section{IncuCyte Analysis}

BMDMs were seeded at a concentration of $1 \times 10^{6}$ cells onto 12 -well plates. The next day, BMDMs were stimulated and incubated with $100 \mathrm{nM}$ of cell-impermeable DNA-binding fluorescent dye Sytox Green (S7020, Life Technologies) to stain dead cells. Cell death was monitored in real-time using a two-color IncuCyte Zoom inincubator imaging system (Essen Biosciences). Nuclear-ID (ENZ-52406, Enzo Life Sciences) was added at the last time point to stain live and dead cells for the total cell number [93]. Resulting images were analyzed using IncuCyte S3 software. Cell death was presented as a percentage of Sytox Green ${ }^{+}$cells to the total cell number. Experiments were conducted with three replicates for each experimental condition and nine image fields per well.

\section{Flow Cytometry}

For intracellular cytokine staining, cells were processed as previously described [94]. The following monoclonal antibodies were diluted 1:300 and used for flow cytometry cellular analyses: CD3 (17A2), CD4 (RM4-5), CD11b (M1/70), CD19 (1D3), CD8a (53-6.7), IFN- $\gamma$ (XMG1.2) and IL-17A (eBio17B7) from eBioscience, CD4 
(GK1.5), TCR- $\beta$ (H57-597), CD11c (N418), Gr1 (RB6-815), F4/80 (BM8), IL-6 (MP520F3) and TNF (MP6-XT22) from BioLegend and CD8a (53-6.7) from Sony. Flow cytometry data were acquired on FACSCalibur (BD) and were analyzed with FlowJo software (FlowJo, LLC and Illumina, Inc).

\section{Statistical Analysis}

GraphPad Prism software was used for data analysis. Data are shown as mean \pm SEM. Statistical significance was determined by performing $t$ tests (two-tailed), one-way ANOVA or log-rank test. $\mathrm{P}<0.05$ was considered to be statistically significant where $* \mathrm{p}<$ $0.05, * * \mathrm{p}<0.01, * * * \mathrm{p}<0.001$, and $* * * * \mathrm{p}<0.0001$. 


\section{CHAPTER 3. STING MEDIATES PROTECTION AGASINT COLON CANCER BY REGULATING COLON INFLAMMATION*}

\section{Introduction}

Colon cancer is the third most common malignant tumor in the United States, with 1.5 million new cases being diagnosed each year [95]. Clinical studies have shown that patients diagnosed with inflammatory bowel diseases have a greater risk of developing colon cancer compared to healthy individuals [96]. Pattern recognition receptors are key initiators of inflammation, which have been shown to be involved in colitis-associated colon cancer development [97].

Cytosolic nucleic acid receptors mediate the detection of DNA and RNA molecules within the cell and provide robust host immune defense against intracellular pathogens and protection against autoimmune diseases [98]. STING is a cytoplasmic receptor for cyclic dinucleotides and an adaptor for other DNA sensors $[12,15,17,19$, 99, 100]. The upstream DNA sensors, which signal through STING include cGAS, IFI16 and DDX41 [12, 99, 100], highlighting an important function for STING in governing multiple DNA recognition pathways. STING recruits TBK1 to activate IRF3, the latter of which translocates into the nucleus in a dimeric form and induces transcription of type I interferons.

Since its discovery, STING has been shown to contribute to the host defense against viral, bacterial and eukaryotic pathogens [101]. Whether STING plays a role during the development of colon cancer or other types of cancer is unknown. Evidence for a role of a number of nucleic acid sensors in tumorigenesis is emerging. Our group and others have previously shown that nucleotide-binding domain, leucine-rich repeat containing protein 3 (NLRP3), a proposed sensor of RNA or DNA:RNA hybrid in the cytoplasm of a cell [102-104], mediates protection against colon cancer development by modulating IL-18 production [27, 29]. The double-stranded DNA sensor, AIM2, and the double-stranded RNA sensor, RIG-I, are downregulated in patients with colorectal cancer and hepatocellular carcinoma, respectively, and reduced expression of these proteins are associated with increased mortality in these patients [26, 105]. Expression of LGP2, a negative regulator of RIG-I, promotes the survival of colon tumor and other cancer cell lines [106]. In addition, mice lacking the DNA sensor, Ku70, in combination with the $\mathrm{p} 53^{\mathrm{R} 172 \mathrm{P}}$ mutation, develop spontaneous colonic inflammation and colon cancer [107]. These studies indicate that nucleic acid sensors may play a critical function in the regulation of tumorigenesis.

\footnotetext{
* Adapted with permission. Zhu Q., Man S.M., Gurung P., Liu Z., Vogel P., Lamkanfi M., Kanneganti T.D. (2014). Cutting Edge: STING mediates protection against colorectal tumorigenesis by governing the magnitude of intestinal inflammation. Journal of Immunology. 193(10):4779-82. Copyright 2014. The American Association of Immunologists, Inc.
} 
In this study, we show that STING-deficient mice (Sting $\left.{ }^{\mathrm{gt} / \mathrm{gt}}\right)$ are highly susceptible to tumor formation in a model of colitis-associated colorectal cancer. The absence of STING leads to excessive colon inflammation during early stages of tumor development, characterized by elevated levels of colonic and circulating proinflammatory cytokines and an increased number of proliferative intestinal epithelial cells. In addition, we observe increased phosphorylation of NF- $\kappa \mathrm{B}$ and STAT3 in STING-deficient mice, indicating a role for STING in governing the suppression of transcription factor activities during intestinal inflammation. Overall, these results underscore a critical role for STING in mediating host protection against colorectal tumorigenesis.

\section{Results and Discussion}

STING is expressed in a variety of tissues and organs in mice (Figure 3-1A). The spleen and small and large intestines expressed the highest levels of STING mRNA (Figure 3-1A). To investigate the role of STING in colon tumor development, we utilized a colitis-associated colon cancer model to induce colon tumorigenesis in mice. We intraperitoneally injected AOM into WT and Sting ${ }^{\mathrm{gt} / \mathrm{gt}}$ mice followed by three rounds of DSS treatment. After 80 days post-AOM injection, we observed that Sting ${ }^{\text {gt/gt }}$ mice had significantly increased tumor burden compared to WT controls (Figure 3-1B-C). Although the number of tumors doubled in Sting ${ }^{\mathrm{g} / \mathrm{gt}}$ mice, the size of tumors in Sting ${ }^{\mathrm{gt} / \mathrm{gt}}$ and WT mice was similar (Figure 3-1D). Histological analysis revealed that Sting ${ }^{\mathrm{gt} / \mathrm{gt}}$ mice exhibited more severe pathological damage after tumor development, characterized by increased colonic inflammation and hyperplasia (Figure 3-1E-G). Histological observations were quantified and these scores revealed that Sting ${ }^{\mathrm{gt} / \mathrm{gt}}$ mice showed enhanced intestinal pathology compared to WT mice (histological scores: $17.6 \pm 1.2$ for Sting $^{\text {gt/gt }}$ and $10.6 \pm 0.9$ for WT; Figure 3-1F). Notably, the incidence of dysplasia was observed exclusively in Sting ${ }^{\text {gt } / \text { gt }}$ mice, with $75 \%$ of the colon samples from Sting ${ }^{\text {gt/gt }}$ mice showing hallmarks of low grade (50\% of the samples) or high grade $(25 \%)$ dysplasia (Figure 3-1H). Therefore, these data suggest a crucial role for STING in suppressing colorectal tumorigenesis in colitis-associated colon cancer.

We observed that Sting ${ }^{\text {gt/gt }}$ mice lost significantly more body weight than WT mice (Figure 3-2A). Indeed, the colon length was greatly reduced in Sting ${ }^{\mathrm{gt} / \mathrm{gt}}$ mice on Day 14 (Figure 3-2B). H\&E staining revealed an increase in cellular infiltration, crypt thickness and hyperchromatin in colon tissues of Sting ${ }^{\text {gt/gt }}$ mice on Day 14 (Figure 3-2C). In agreement, all of the histologic parameters assessed - inflammation, ulceration and hyperplasia - were significantly higher in Sting ${ }^{\text {gt/gt }}$ mice compared to WT mice

(Figure 3-2D-E). To assess whether STING mediates neoplastic changes that predispose the host to increased tumorigenic events, we performed Ki-67 staining on colon tissue sections and quantified the magnitude of intestinal epithelial proliferation. In line with our histological analysis, the colon of Sting ${ }^{\mathrm{gt} / \mathrm{gt}}$ mice had significantly increased numbers of Ki-67 positive cells per crypt $(58.7 \pm 2.1 ; \mathrm{n}=137)$ compared to WT mice $(39.6 \pm 1.3$; $\mathrm{n}=153 ; \mathrm{P}<0.0001$ ) on Day 14 (Figure 3-2F). Taken together, these results suggest that 


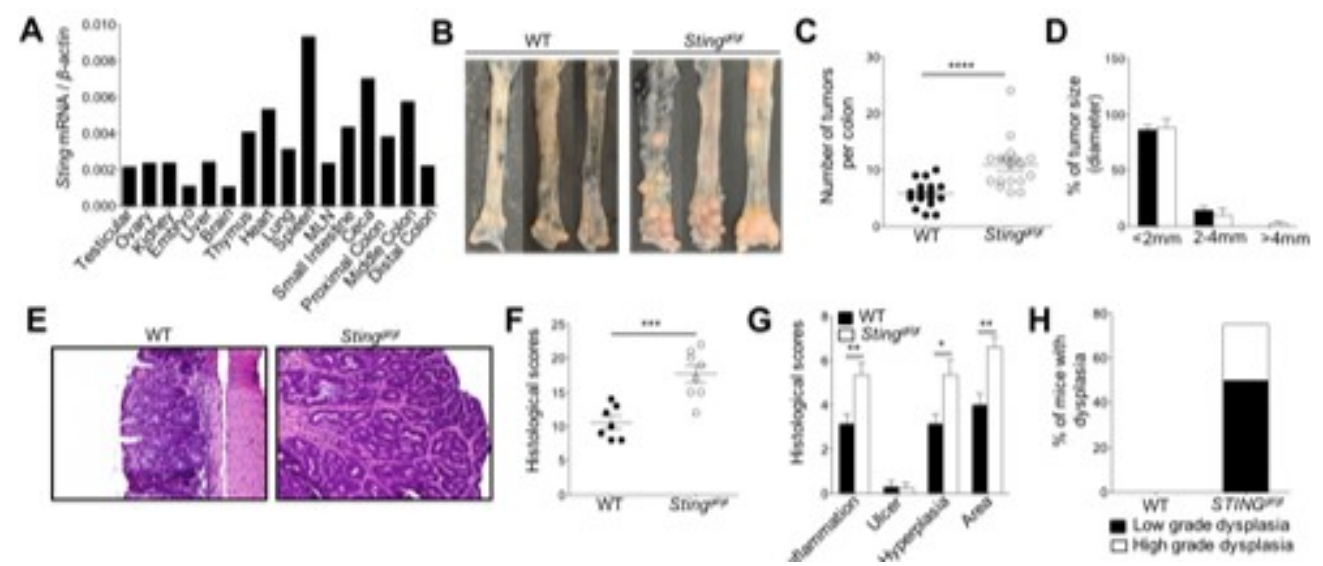

Figure 3-1. STING is critical for mediating protection against colon tumor development.

(A) Relative Sting mRNA levels in different tissues of WT mice. (B) Colons from WT and Sting ${ }^{g t / g t}$ mice on Day 80 post-AOM injection. (C) Tumor number and (D) size in WT and Sting ${ }^{g t / g t}$ mice. (E) Colon tissue sections by H\&E staining. Magnification: $20 \times$. (F) Total histological scores and $(\mathrm{G})$ scores for different parameters on Day 80. $(\mathrm{H})$ Percentage of WT and Sting ${ }^{g t / g t}$ mice with dysplasia. ${ }^{*} \mathrm{p}<0.05 ; *{ }^{*} \mathrm{p}<0.01 ; * * * \mathrm{p}<0.001$; $* * * * \mathrm{p}<0.0001$. (two-tailed $\mathrm{t}$ test $[\mathrm{B}-\mathrm{D}, \mathrm{F}$, and $\mathrm{G}]$ ). Data are from one experiment representative of three independent experiment $(\mathrm{B}$ and $\mathrm{D}-\mathrm{H})$ or pooled from three independent experiments (A and C; mean and SEM in C, D, F and G). 

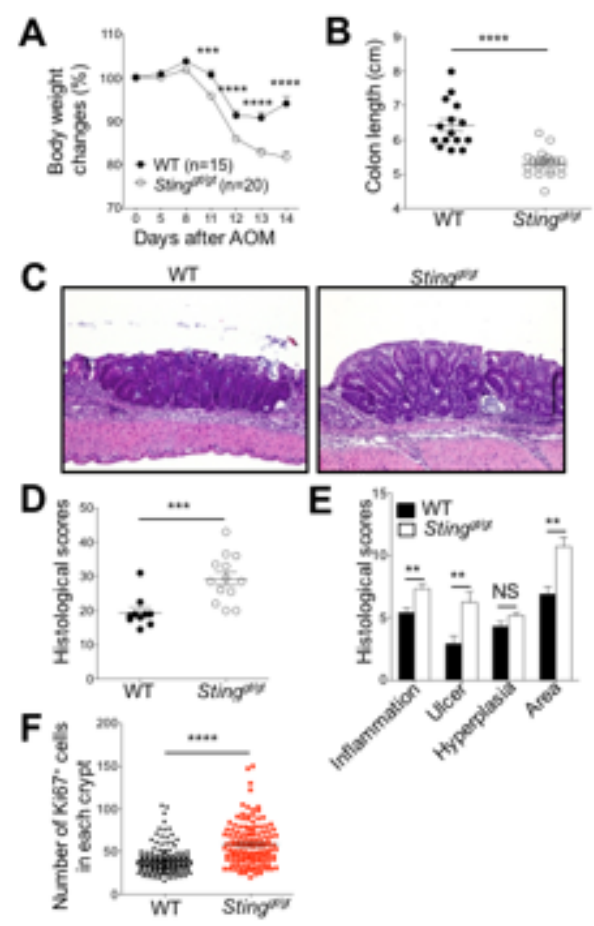

Figure 3-2. STING dampens early intestinal damage and overt proliferation. (A) Body weight changes in WT and Sting ${ }^{\text {gt/gt }}$ mice during early stages of colorectal tumorigenesis. (B) Colon length on Day 14 post-AOM injection. (C) H\&E staining of colon tissue sections on Day 14. Magnification: 10×. (D) Total histological scores and (E) scores for different parameters on Day 14. (F) The number of Ki-67-positive cells in each crypt of each animal on Day 14 (at least 27 crypts per mouse). WT n=5; Sting ${ }^{\text {gt/gt }}$ $\mathrm{n}=5$. Data are mean \pm SEM of two independent experiments. NS, not statistically significant; $* * \mathrm{p}<0.01 ; * * * \mathrm{p}<0.001 ; * * * * \mathrm{p}<0.0001$. (two-tailed t test $[\mathrm{A}, \mathrm{B}, \mathrm{D}, \mathrm{E}$ and $\mathrm{F}]$ ). Data are from one experiment representative of two independent experiment $(C, D, E$ and F) or pooled from two independent experiments (A and B; mean and SEM in A, B, D, E and F). 
STING plays an important function in controlling disease initiation and susceptibility to hyper-proliferation during early stages of colitis-associated colon cancer.

Since inflammation is one of the most important drivers in the development of colon cancer [97], we hypothesized that STING is controlling inflammation during early stages of AOM-DSS treatment, which ultimately determines host susceptibility to colorectal tumorigenesis. To analyze the magnitude of colonic inflammation in Sting ${ }^{\mathrm{gt} / \mathrm{gt}}$ mice, we measured levels of the pro-inflammatory cytokines IL-6 and CXCL1 (KC) on Day 14 post-AOM injection. We observed a significant increase in the levels of IL-6 and $\mathrm{KC}$ in colon tissues of Sting ${ }^{\text {gt/gt }}$ mice compared to WT mice (Figure 3-3A). We confirmed this finding and observed elevated levels of circulating IL-6 and $\mathrm{KC}$ in the serum of Sting ${ }^{\mathrm{gt} / \mathrm{gt}}$ mice (Figure 3-3B). Consistently, we found elevated phosphorylation of ERK and IאB $\alpha$ in colon tissues of Sting ${ }^{\mathrm{gt} / \mathrm{gt}}$ mice compared to WT mice on Day 14 (Figure 3-3C). Previous reports have shown that myeloid cell-derived IL-6 signals through IL-6R and gp130 to activate cytoplasmic STAT3 in intestinal epithelial cells $[108,109]$. STAT3 is frequently activated in pre-malignant and cancer cells to promote colon tumorigenesis by amplifying inflammation and tumorigenic transformation [110]. Indeed, colon tissues from Sting ${ }^{\text {gt/gt }}$ mice showed increased STAT3 phosphorylation compared to WT mice (Figure 3-3D). We also measured production of type I IFN by testing the level of IFN- $\beta$ after 14 days post-AOM injection and detected only basal levels of IFN- $\beta$ in colon tissues from WT or Sting ${ }^{\text {gt/gt }}$ mice (Figure 3-4A).

Caspase-1-mediated release of IL-18 following inflammasome activation has been shown to play a protective role in preventing AOM-DSS-induced colitis and colitisassociated colorectal tumorigenesis [27-29, 111]. Mice lacking caspase-1 or IL-18 exhibited elevated tumor burden in the colon with increased levels of pro-inflammatory cytokines during AOM-DSS-induced colon tumorigenesis [29]. We found reduced levels of caspase-1 activation and IL-18 release in colon tissues of Sting $\mathrm{g}^{\mathrm{g} / \mathrm{gt}}$ mice compared to WT mice after 14 days (Figure 3-4B-C). Therefore, it is possible that the crosstalk between STING and caspase-1 could mediate protection against colon tumorigenesis at the intestinal barrier. Indeed, recent studies suggest that activation of the STING pathway leads to assembly of NLRP3 inflammasome [112,113], potentially by inducing lysosomal cell death and potassium efflux [112]. It is possible that STING induces NLRP3 inflammasome activation in the colon leading to caspase-1-mediated protective IL-18 release. These findings highlight that STING restricts NF-kB and STAT3 signaling pathways by activating inflammasome responses to control colon inflammation and tumorigenesis.

The gut microbiota plays a critical role in modulating susceptibility to colorectal cancer. Dysbiosis is associated with increased susceptibility to colitis and colon cancer development [97]. To investigate whether an altered gut microbiota profile contributed to the elevated incidence of colorectal tumorigenesis in Sting ${ }^{\mathrm{gt} / \mathrm{gt}}$ mice, we performed realtime qPCR analysis to detect the abundance of five major intestinal bacterial populations from WT and Sting ${ }^{\mathrm{gt} / \mathrm{gt}}$ mice. We found that WT and Sting ${ }^{\mathrm{gt} / \mathrm{gt}}$ mice harbored similar levels of Prevatollaceae, Bacteroides, mouse intestinal Bacteroides (MIB), segmented filamentous bacteria (SFB) and TM7 (Figure 3-4D-H). Therefore, it is unlikely that 


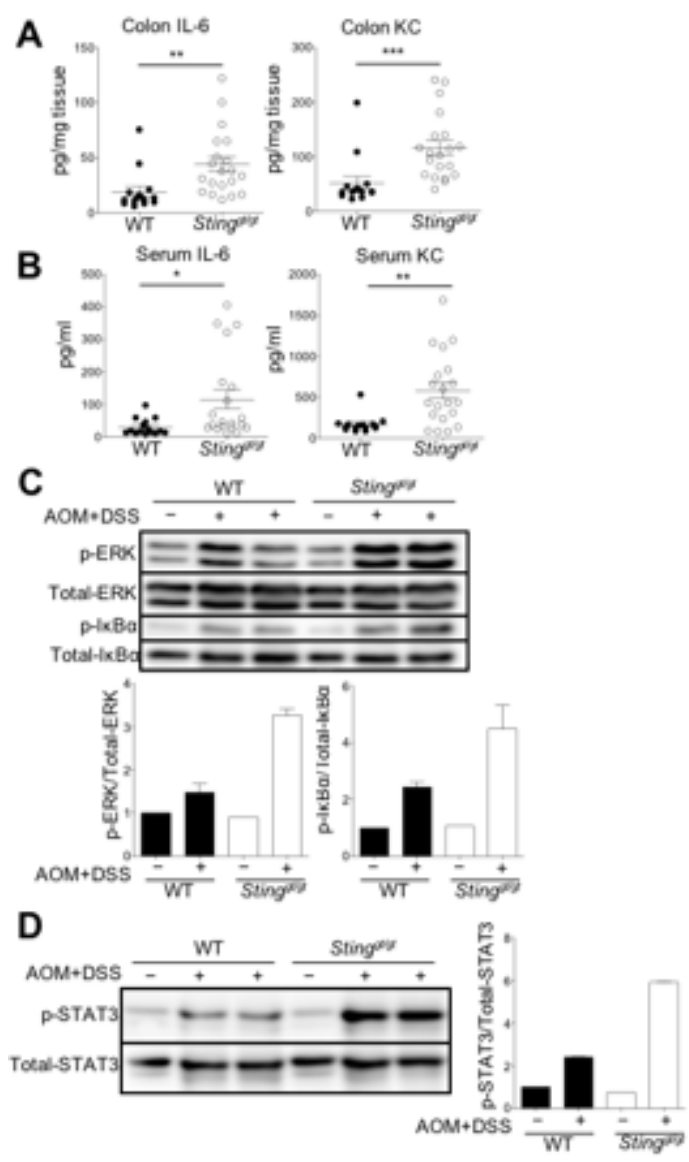

Figure 3-3. STING suppresses overt colon inflammation during tumor development.

(A and B) Levels of IL-6 and KC in colon tissues (A) and serum (B) on Day 14 postAOM injection. (C and D) Phosphorylation of ERK, IкBa (C) and STAT3 (D) in colon tissues on Day 14, quantified by densitometric analysis. Data are mean $\pm \mathrm{SEM}$ of two independent experiments. ${ }^{*} \mathrm{p}<0.05 ; * * \mathrm{p}<0.01 ; * * * \mathrm{p}<0.001$. (two-tailed t test [A and B]). Data are from one experiment representative of two independent experiment ( $C$ and $D)$ or pooled from two independent experiments (A and B; mean and SEM in A-D). 

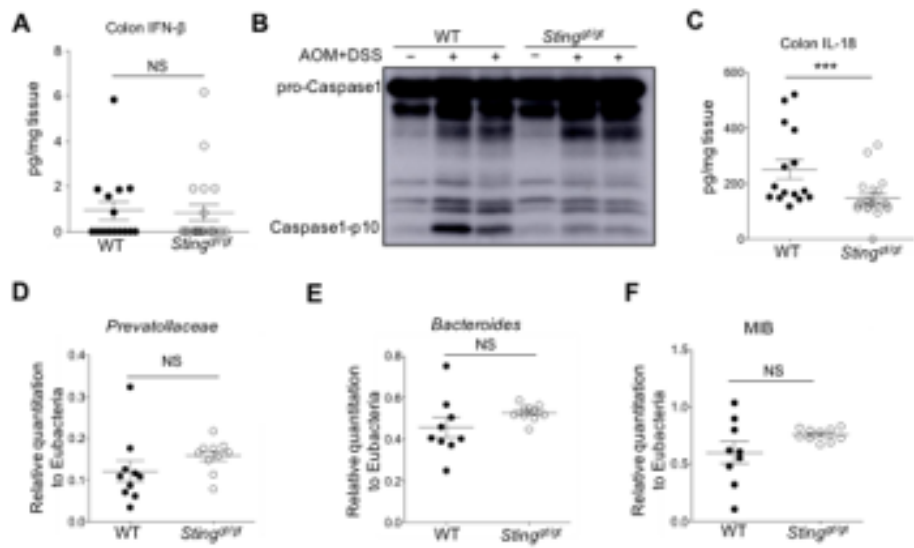

E

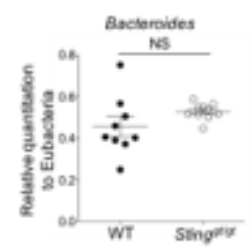

$\mathbf{F}$

G

H
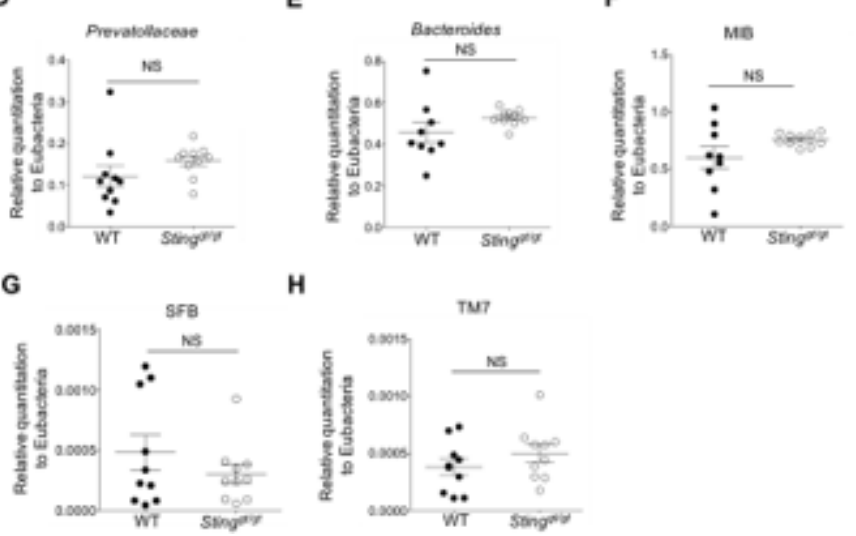

Figure 3-4. The type I IFNs, caspase-1 and gut microbiota profile of WT and Sting ${ }^{g t / g t}$ mice.

(A) Levels of IFN- $\beta$ in colon tissues from WT and Sting ${ }^{g t / g t}$ mice after 14 days post-AOM injection were measured by ELISA. (B) Caspase-1 cleavage in colon lysates from WT and Sting ${ }^{g t / g t}$ mice on Day 14. (C) Level of IL-18 in colon tissues on Day 14. (D-H) Levels of Prevatollaceae, Bacteroides, MIB, SFB and TM7 in untreated WT and Sting ${ }^{\text {gt/gt }}$ mice. Data are shown as mean \pm SEM of two independent experiments. NS, not statistically significant; $* * * p<0.001$. 
differences in these bacterial populations contributed to the protective role of STING during colon cancer development.

During AOM-DSS-induced colon tumorigenesis, STING itself or in concert with an upstream DNA sensor may recognize either cytosolic DNA in tumor cells, or DNA upon DNA damage [114], or DNA derived from dying cells during intestinal barrier damage or colon tumor cells [115]. In addition, oxidative DNA damage has been shown to accumulate in intestinal epithelial cells and activate STING signaling $[116,117]$. It is possible that recognition of self-DNA by STING mediates inflammasome activation, which suppresses colon inflammation and reduces the likelihood of uncontrolled inflammation leading to tumorigenesis.

Overall, our results underscore a novel role for STING in modulating activation of NF- $\kappa$ B and STAT3 signaling and production of IL-18 during the development of colon cancer. These inflammatory mediators may modulate the tumorigenic properties of intestinal cells, which ultimately regulate proliferation and tumorigenesis. Collectively, our data indicate that STING plays a critical role in mediating protection against inflammation-associated colon cancer development. Therapeutic modulation of STING in susceptible individuals may have the potential to prevent and treat colon cancer. 


\section{CHAPTER 4. ROLE OF THE DNA SENSOR AIM2 IN COLON CANCER*}

\section{Introduction}

Colorectal cancer is a leading cause of cancer-related deaths, with 160,000 cases being diagnosed annually in the USA [118]. Mutations in the gene encoding AIM2 are frequently identified in patients with colorectal cancer. A previous study has shown that over $50 \%$ of tumors from patients with small bowel cancer have frameshift mutations in the gene encoding AIM2 [119]. Further genetic evidence has shown that frameshift and missense mutations are targeted to the coding regions of the gene encoding AIM2 in colon cancer tissues and cell lines [38,39]. These include frameshift mutations in the A10 repeat of exon 6 , a deletion mutation in the A6 repeat of exon 5, and a nonsynonymous nucleotide exchange within exon 2 of the gene encoding AIM2 - all of which were identified in tumor tissues of patients with colorectal cancer with a high level of microsatellite instability associated with deficiency in mismatch repair [38]. Analysis of 414 colorectal tumors and matching control tissues further revealed that $67 \%$ of the tumors displayed reduced expression of the gene encoding AIM2 relative to control tissues [26]. Nearly $50 \%$ of patients who completely lacked expression of the gene encoding AIM2 in their tumor cells die from their cancer within five years of diagnosis, whereas over $70 \%$ of patients whose tumor cells retained some expression survived beyond five years after their diagnosis [26]. A better understanding of the biological function of AIM2 in colorectal cancer is key to identifying therapies that could be used in the treatment of this disease.

AIM2 is a cytosolic dsDNA receptor which contributes to the host defense against bacterial and viral pathogens, such as Francisella tularensis and vaccinia virus, respectively [32-35, 41, 42]. We previously identified that infection by the cytosolic bacterium $F$. novicida activates the transcription factor IRF1, which induces production of interferon-inducible GTPases called GBPs [44]. GBPs attack and kill bacteria in the cytoplasm, resulting in the liberation of bacterial DNA for recognition by AIM2 [44, 45]. Upon binding of dsDNA in the cytoplasm of infected cells, AIM2 recruits the adaptor protein ASC to assemble an inflammasome complex that activates caspase-1, a cysteine protease that induces pyroptosis and mediates the cleavage of the inflammatory cytokines IL-1 $\beta$ and IL-18. Structural analysis of AIM2 revealed that the HIN200 domain binds dsDNA, whereas the pyrin domain recruits ASC [120]. DNA accumulated in keratinocytes also activates the AIM2 inflammasome to drive the release of IL-1 $\beta$ in lesions of patients with psoriasis [121], suggesting that AIM2 has the capacity to recognize damage-associated molecular patterns released by the cell. Activation of AIM2

* Adapted with open access permission. Man S.M.*, Zhu Q.*, Zhu L.*, Liu Z.*, Karki R., Malik A., Sharma D., Li L., Malireddi R.K., Gurung P., Neale G., Olsen S.R., Carter R.A., McGoldrick D.J., Wu G., Finkelstein D., Vogel P., Gilbertson R.J., Kanneganti T.D. (2015). Critical role for the DNA sensor AIM2 in stem cell proliferation and cancer. Cell. 162(1):45-58. (* Co-first author) 
must, therefore, be tightly regulated to allow clearance of pathogens while maintaining homeostasis to prevent the development of autoimmune conditions.

In this study, we found that AIM2-deficient $\left(\operatorname{Aim}^{-/-}\right)$mice developed more tumors in the colon following AOM and DSS-induced colitis-associated tumorigenesis. Unlike its role in the host defense against infection, AIM2 protected against tumorigenesis by controlling intestinal epithelial cell proliferation via a mechanism independent of inflammasomes and inflammatory mediators. Upon dyregulated Wnt signaling, AIM2 suppressed expansion of tumor-initiating intestinal stem cells lining the base of the crypt. Remarkably, reciprocal exchange of the gut microbiota between healthy WT mice and $\mathrm{Aim} 2^{-/-}$mice reduced the susceptibility of $\mathrm{Aim} 2^{-/-}$mice to colorectal tumorigenesis. These findings revealed a role for AIM2 and its synergy with gut microbiota in the development of colorectal cancer.

\section{Results}

\section{The DNA Receptor AIM2 Is Required to Prevent Colorectal Cancer}

Mutations in the gene encoding AIM2 are associated with colorectal cancer and reduced expression of this gene is linked to increased mortality in humans [122-125]. In mice, the gene encoding AIM2 was widely expressed in the intestine, especially in the colon (Figure 4-1A), which suggested a role for AIM2 in regulating homeostasis in the gastrointestinal tract. To investigate the role of AIM2 in colorectal cancer, we used an established model of colitis-associated colorectal tumorigenesis whereby mice were injected intraperitoneally with AOM, followed by three rounds of DSS treatment (Figure 2-1). Expression of the gene encoding AIM2 was drastically reduced in the colon of WT mice 14 or 80 days after injection of AOM (Figure 4-1B-C).

We treated cohorts of WT and Aim $2^{-/-}$mice with AOM followed by DSS and examined the development of colon tumors after 80 days. We found that $\mathrm{Aim} 2^{-/-}$mice developed significantly increased numbers and size of tumors throughout the middle and distal colon compared with WT mice (Figure 4-1D-F). Aim $2^{-/-}$mice also tended to lose more body weight over the course of DSS treatment after injection of AOM

(Figure 4-1G). Histopathological analysis revealed that $\mathrm{Aim} 2^{-/-}$mice exhibited increased hyperplasia in the proximal, middle and distal colon (Figure 4-1H). Low-grade dysplasia was restricted to tumor-bearing WT mice whereas both low- and high-grade dysplasia was observed in Aim $^{-/-}$mice (Figure 4-1I-J). These results suggested that AIM2 mediated protection against colorectal tumorigenesis.

\section{AIM2 Controls Tumor Development Independently of Inflammasomes}

AIM2 is a dsDNA receptor that contributes to the host defense against bacterial and viral pathogens by inducing inflammasome responses, including the production of 

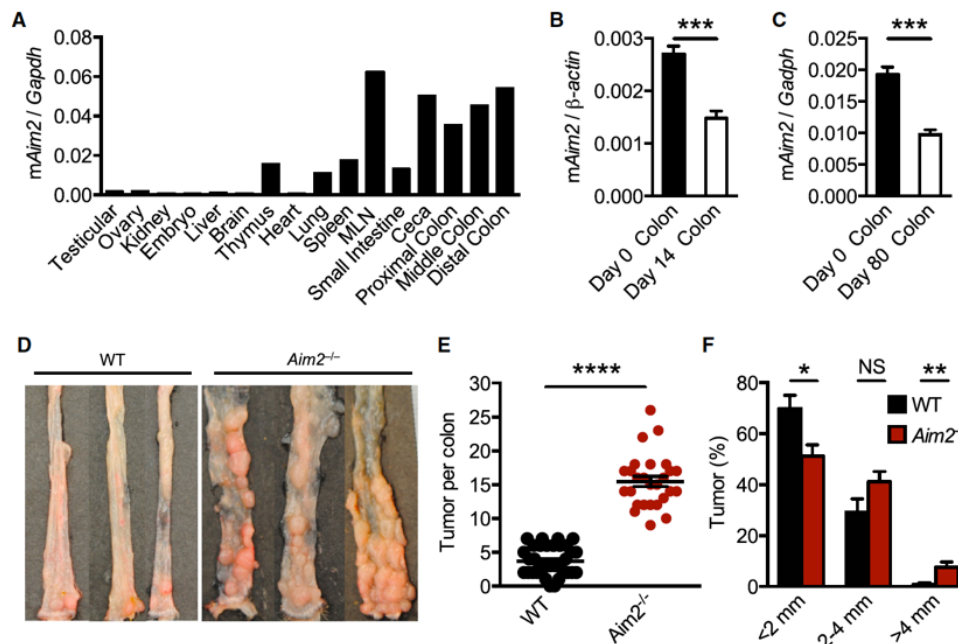

$\mathbf{F}$
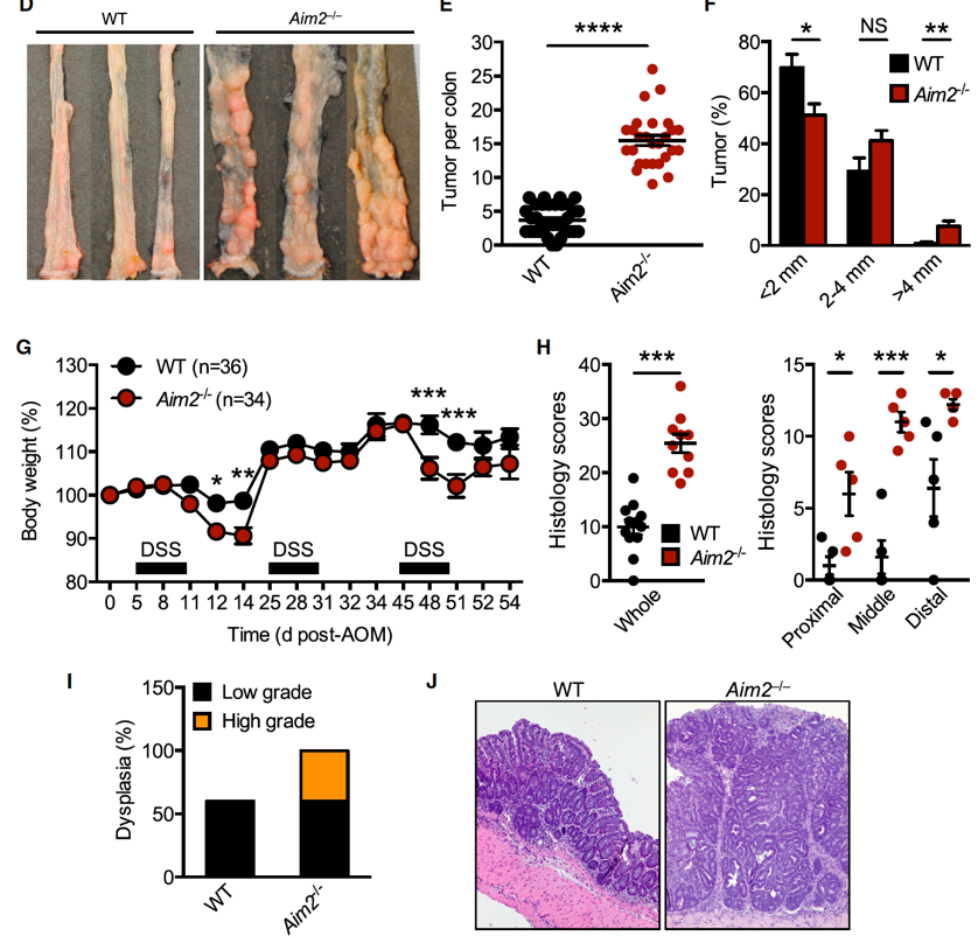

$\mathbf{J}$

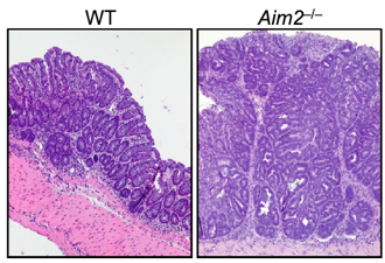

Figure 4-1. AIM2 prevents colitis-associated colorectal tumorigenesis.

(A) Relative expression of the gene encoding AIM2 in tissues of WT mice. (B) Relative expression of the gene encoding AIM2 in colon tissues of untreated WT mice or WT mice 14 days after injection of AOM. (C) Relative expression of the gene encoding AIM2 in colon tissues of untreated WT mice or WT mice 80 days after injection of AOM. (D) Colon tumors in WT and Aim $2^{--}$mice 80 days after injection of AOM. (E and F) Number and size of colon tumors in WT $(\mathrm{n}=34)$ and $\operatorname{Aim}^{-/-}(\mathrm{n}=27)$ mice. $(\mathrm{G})$ Body weight change of mice pooled from six independent experiments. $(\mathrm{H})$ Histological scores 80 days after injection of AOM. (I) Percentages of mice with dysplasia 80 days after injection of AOM. (J) H\&E staining of colon tumors. Original magnification, $10 \times .{ }^{*} \mathrm{p}<$ $0.05 ; * * \mathrm{p}<0.01 ; * * * \mathrm{p}<0.001 ; * * * * \mathrm{p}<0.0001$; NS, not statistically significant (twotailed $t$ test $[B, C, E, F$, and $H]$ or one-way ANOVA [G]). Data are from two (A-C) or six independent experiments (D-J; mean and SEM in B, C, E, F, G, and H). 
IL-18. We and others have previously shown that IL-18 induced by the related inflammasomes, NLRP3 or PYRIN inflammasome, is required for protection against colitis and colitis-associated tumorigenesis [27-29, 111]. To investigate the molecular mechanism underpinning AIM2-dependent protection against colorectal tumorigenesis, we first examined whether there was a lack of inflammasome signaling in $\mathrm{Aim}^{-/-}$mice that could be responsible for their increased susceptibility to tumorigenesis. We analyzed the levels of caspase-1 processing in the colon of WT and Aim ${ }^{-/}$mice and found similar levels of caspase-1 activation 14 days after injection of AOM (Figure 4-2A). We also detected similar levels of IL-18 in the colon tissues and serum of untreated WT and Aim $2^{-/}$mice or in WT and Aim $2^{-/-}$mice 8, 14 or 80 days after injection of AOM (Figure 4-2B). To further confirm these results, we measured IL-1 $\beta$ and found similar levels of this inflammasome-dependent cytokine in the colon tissues of untreated WT and $\mathrm{Aim}^{-/-}$mice or in WT and $\mathrm{Aim}^{-/-}$mice 14 or 80 days after injection of AOM (Figure 4-2C).

Although AIM2 functioned independently of the inflammasome in protection against colonic tumorigenesis, we investigated whether other inflammatory mediators might be playing a role in the absence of AIM2 that could have accelerated the progression of colorectal cancer. Shortening of the colon during DSS treatment is a hallmark of inflammation. However, we observed similar colon lengths in WT and Aim2 ${ }^{-}$mice 14 or 80 days after injection of AOM (Figure 4-2D). The NF-kB pathway is a major driver of inflammation. To this end, we analyzed phosphorylation of ERK and IKB $\alpha$ in colon tissues of WT and Aim2 $2^{--}$mice following AOM-DSS treatment. Both groups of mice exhibited similar levels of phosphorylated ERK and IкB $\alpha$ (Figure 4-2E). In agreement, the levels of pro-inflammatory cytokines IL-6, TNF and G-CSF and the chemokine $\mathrm{KC}$ were not significantly increased in $\mathrm{Aim}^{-/-}$mice 14 days after injection of AOM when compared with WT mice (Figure 4-2F). However, an increase in the levels of TNF, G-CSF and KC were observed 80 days after injection of AOM (Figure 4-2F). We further assessed the levels of cytokines IL-22, IL-23p19, IFN- $\beta$, IFN- $\gamma$ and IL-17A. Using qRT-PCR analysis, we found that the expression of genes encoding these cytokines was similar in colon tissues of untreated WT and $\mathrm{Aim} 2^{-/-}$mice and in colon tissues of WT and $\mathrm{Aim}^{-/-}$mice 14 days after injection of AOM (Figure 4-3A-B). However, Aim $^{-/-}$mice had an increase in the expression of the gene encoding IL-10 14 days after injection of AOM (Figure 4-3C). Furthermore, we found that the expression of genes encoding TGF- $\beta 1$ and TGF- $\beta 2$ was similar in colon tissues of untreated WT and $\mathrm{Aim}^{-/-}$mice and in WT and $\mathrm{Aim}^{-/-}$mice 14 days after injection of AOM (Figure 4-3C).

We used an automated hematology analyzer to determine the number and influx of circulating white blood cells from the peripheral blood of WT and $\mathrm{Aim}^{-/-}$mice. We observed similar levels of neutrophils, lymphocytes, monocytes, eosinophils and basophils and total white blood cells in the blood of WT and $\mathrm{Aim}^{-/-}$mice 14 or 80 days after injection of AOM (Figure 4-3D). While there was an increase in circulating lymphocytes in $\mathrm{Aim}^{-/-}$mice on day 80 compared with day 14, the proportion of IFN- $\gamma-$, IL-17- or TNF-expressing $\mathrm{CD}^{+}$or $\mathrm{CD} 8^{+} \mathrm{T}$ cells in the MLNs and spleens was similar in WT and Aim $^{-/-}$mice (Figure 4-2G). In addition, the prevalence of splenic and MLNassociated CD1 $1 \mathrm{~b}^{+}$cells producing TNF or IL-6 was also similar in WT and $\mathrm{Aim}^{-/-}$mice 

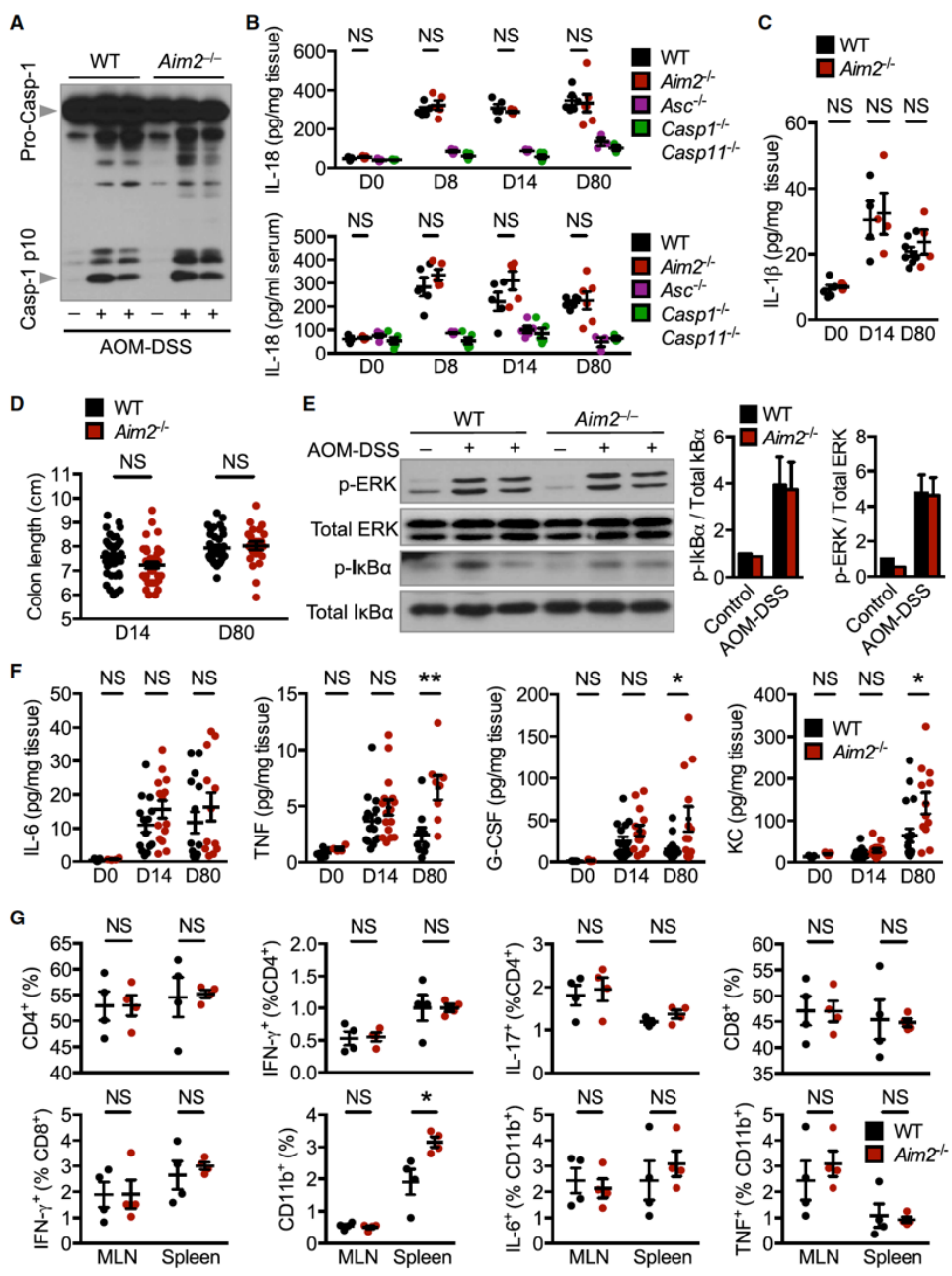

Figure 4-2. AIM2 governs colorectal tumorigenic susceptibility independently of inflammasomes and a number of inflammatory mediators.

(A) Caspase-1 processing in colon lysates from WT and $\mathrm{Aim} 2^{-1-}$ mice 14 days after injection of AOM. (B) Levels of IL-18 in the colon tissues and sera. (C) Levels of IL-1b in colon tissues. (D) The lengths of colon from WT and $A \mathrm{im} 2^{-/-}$mice 14 days after injection of AOM. (E) Immunoblot analysis (left) and densitometric quantification of band intensity of phosphorylated and total ERK and IкBa (right) in colon tissues of WT and $\mathrm{Aim}^{-/-}$mice 14 days after injection of AOM. (F) Levels of IL-6, TNF, G-CSF, and $\mathrm{KC}$ in colon tissues. (G) Percentages of $\mathrm{CD}^{+}$cells, $\mathrm{CD}^{+}$cells expressing IFN- $\gamma$ or IL$17, \mathrm{CD}^{+}$cells, $\mathrm{CD} 8^{+}$cells expressing IFN- $\gamma, \mathrm{CD} 11 \mathrm{~b}^{+}$cells, and $\mathrm{CD} 11 \mathrm{~b}^{+}$cells expressing IL-6 or TNF. ${ }^{*} \mathrm{p}<0.05 ; * * \mathrm{p}<0.01$; NS, not statistically significant (two-tailed t test [B$\mathrm{D}, \mathrm{F}$, and $\mathrm{G}]$ ). Data are from one experiment representative of two independent experiment $(\mathrm{A}-\mathrm{C}$ and $\mathrm{G}$ ) or pooled from three independent experiments (D-F; mean and $\mathrm{SEM}$ in $\mathrm{B}-\mathrm{G})$. 

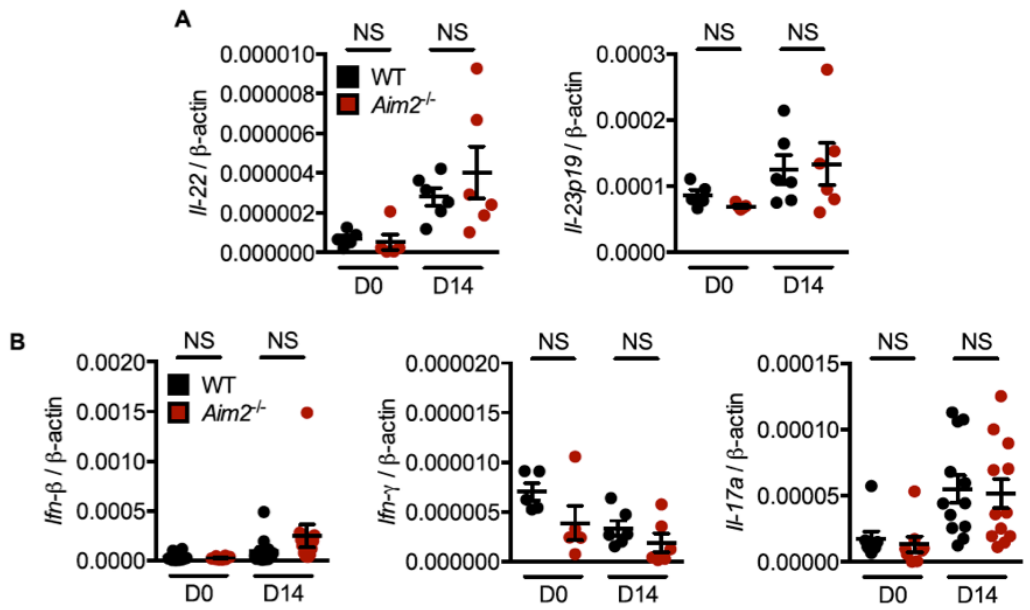

C
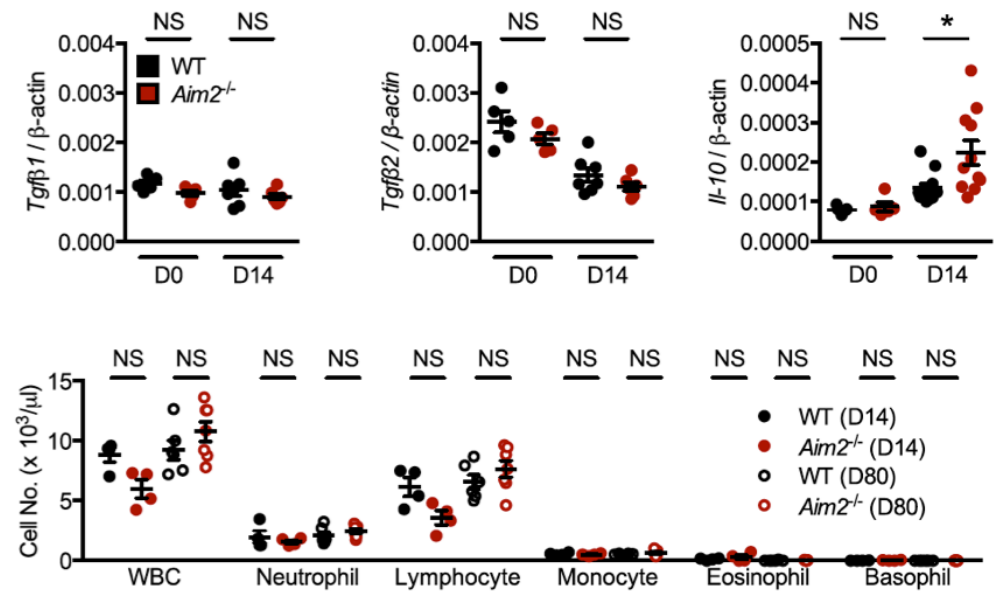

Figure 4-3. AIM2 governs colorectal tumorigenic susceptibility independently of the magnitude of inflammation.

(A) Relative expression of genes encoding IL-22 and IL-23p19 in colon tissues of untreated WT and $\mathrm{Aim}^{-/-}$mice or in WT and $\mathrm{Aim}^{2^{--}}$mice 14 days after injection of AOM. (B) Relative expression of genes encoding IFN- $\beta$, IFN- $\gamma$ and IL-17A in colon tissues of untreated WT and $A i m 2^{-/-}$mice or in WT and $A i m 2^{-/-}$mice 14 days after injection of AOM. (C) Relative expression of genes encoding TGF $\beta 1$, TGF $\beta 2$ and IL-10 in colon tissues of untreated WT and $\mathrm{Aim}^{-/-}$mice or in WT and $\mathrm{Aim} 2^{-/-}$mice 14 days after injection of AOM. (D) Automated hematology analyzer of white blood cell (WBC) populations in the whole blood of untreated WT and Aim $2^{-/}$mice and WT and Aim2 $2^{-/}$ mice 14 or 80 days after injection of AOM. ${ }^{*} p<0.05$; NS, not statistically significant; two tailed $t$ test. Data are from one experiment (D) or pooled from two independent experiments $(\mathrm{A}-\mathrm{C}$, mean and SEM in $\mathrm{A}-\mathrm{D})$. 
(Figure 4-2G). The prevalence of total $C D 11 b^{+}$cells was, however, elevated in the spleen, but not in the MLN. The modest increases in some inflammatory parameters in mice lacking AIM2 suggested a role for an immunological component in shaping tumor development in these mice. Identification of a role for AIM2 independently of classical inflammasome signaling and a number of other inflammatory mediators also suggested, at least in parts, alternative functions for AIM2 in the regulation of gastrointestinal homeostasis during colorectal tumorigenesis.

\section{AIM2 Controls Proliferation of Enterocytes}

Given that the increased susceptibility to tumor development in $A i m 2^{-/-}$mice could not be explained by differences in inflammasome activity or by a number of inflammatory mediators, we asked whether AIM2 regulated cellular proliferation or death following exposure to AOM and DSS. We used immunohistochemistry techniques to identify proliferative cells in the colon of WT and Aim $2^{--}$mice. We observed a significantly higher number of $\mathrm{BrdU}^{+}$proliferative cells per crypt in the colon of $\mathrm{Aim}^{2^{--}}$ mice 14, but not 8, days after injection of AOM (Figure 4-4A). We confirmed these findings and found a significantly higher number of $\mathrm{Ki}^{+} 7^{+}$cells per crypt in the intestinal epithelium of $\mathrm{Aim}^{-/-}$mice 14 days after injection of AOM compared with WT mice, whereas no difference was observed in untreated WT and $A \mathrm{im} 2^{-/}$mice (Figure 4-4A). Further, we used microarray analysis to screen for genes expressed differentially in colon tissues of WT and $\mathrm{Aim} 2^{-/}$mice 14 days after injection of AOM. Using this approach, we indeed identified increased expression of genes encoding molecules involved in cell proliferation in $\mathrm{Aim} 2^{-/-}$mice relative to their expression in WT mice (Figure 4-4B). These included genes encoding S100A9 and BIRC5, which have previously been linked to colorectal carcinoma in humans [126-128]. We confirmed using qRT-PCR analysis an increased expression of the top three differentially expressed genes encoding molecules involved in proliferation, S100A9, SNRPD1 and DBF4, identified in our microarray (Figure 4-4C).

To further investigate the mechanism of unchecked cellular proliferation when AIM2 was absent, we examined activities of the phosphatidylinositol 3-Kinase-AKT pathway, which has a major role in cell proliferation and survival and in development of cancer [129]. We found increased phosphorylation of AKT at Ser473 in Aim $^{-/-}$mice compared with WT mice 14 days after injection of AOM (Figure 4-4D), indicating activation of the AKT pathway. Phosphorylation of AKT at Ser473 was similar between WT and $A i m 2^{-/-}$mice 80 days after injection of AOM (Figure 4-4D). The phosphatidylinositol 3-Kinase-AKT pathway is negatively regulated by the tumor suppressor PTEN [130]. Phosphorylation of PTEN at S380, T382, and T383 maintains PTEN in a relatively inactive state [131]. Indeed, we saw increased phosphorylation of PTEN at S380, T382, and T383 in Aim $2^{-/-}$mice compared with WT mice 14 days after injection of AOM (Figure 4-4D). Increased AKT activation and transcription of genes involved in cell proliferation could explain increased proliferation in the colon of mice lacking AIM2 following damage induced by AOM. Consistent with increased AKT activation, we found an elevated level of the transcription factor and proto-oncogene c- 

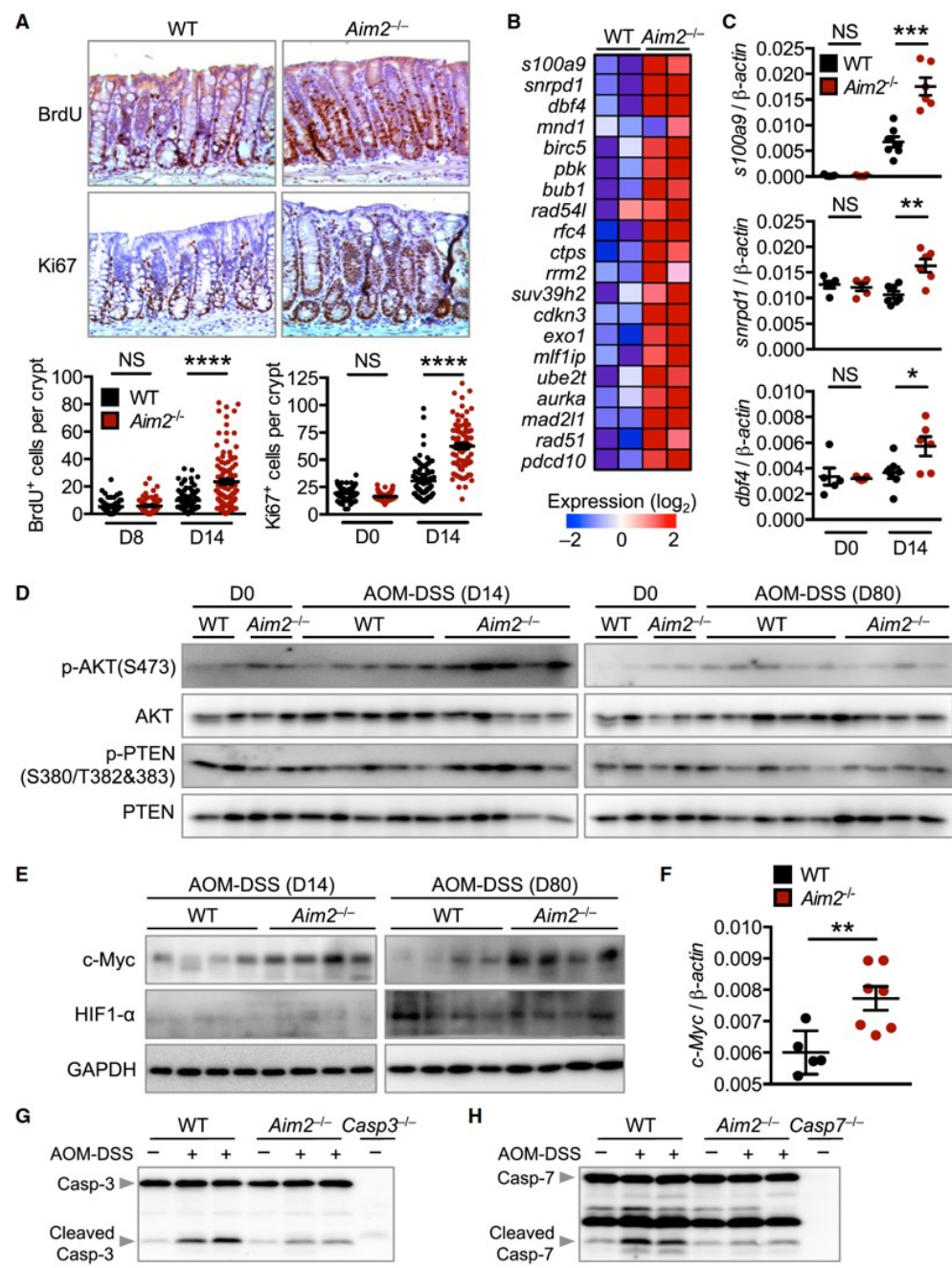

Figure 4-4. AIM2 suppresses overt proliferation.

(A) Quantification of the number of $\mathrm{BrdU}^{+}$cells in each crypt of WT (day 8, $\mathrm{n}=3$; day 14, $\mathrm{n}=5$ ) and $\operatorname{Aim}^{-/-}$(day 8, $\mathrm{n}=4$; day 14, $\mathrm{n}=5$ ) mice. Quantification of the number of $\mathrm{Ki}^{+} 7^{+}$in each crypt of WT (day 0, $\mathrm{n}=4$; day 14, $\mathrm{n}=4$ ) and $\operatorname{Aim}^{-/-}$(day $0, \mathrm{n}=3$; day 14, $\mathrm{n}=4$ ) mice. (B) Microarray analysis of genes encoding molecules involved in proliferation with significantly higher or lower expression in $A i m 2^{-/}$mice 14 days after injection of AOM compared with their WT counterparts. (C) Real-time qRT-PCR analysis of genes encoding S100A9, SNRPD1, and DBF4 in WT and Aim2 ${ }^{-/-}$mice 14 days after injection of AOM. (D and E) Immunoblot analysis of phosphorylated and total AKT and phosphorylated and total PTEN (D) or c-Myc, HIF1-a and GAPDH (loading control; E) from colon tissues. (F) Real-time qRT-PCR analysis of the gene encoding cMyc in colon tissues of mice 14 days after injection of AOM. ( $\mathrm{G}$ and $\mathrm{H}$ ) Immunoblot analysis of caspase-3 $(\mathrm{G})$ and -7 activation $(\mathrm{H})$ in colon tissues of mice 8 days after injection of AOM. At least 20 crypts were counted in each animal (A). ${ }^{*} p<0.05 ; *{ }^{*} p<$ $0.01 ; * * * p<0.001 ; * * * * p<0.0001 ;$ NS, no statistical significance (two-tailed t test [A, $\mathrm{C}$, and F]). Data represent one experiment with two biological replicates per genotype (B) or from one experiment representative of two independent experiments (A, C, and D-H) (mean and SEM in A, C, and F). 
Myc in $\mathrm{Aim}^{-/-}$mice compared with WT mice 14 and 80 days after injection of AOM (Figure 4-4E). Expression of the transcription factor HIF1- $\alpha$, however, was similar between WT and Aim $2^{-/}$mice (Figure 4-4E). We further confirmed by qRT-PCR analysis an increased expression of the gene encoding c-Myc (Figure 4-4F). In addition, increased intestinal cell proliferation was linked to reduced apoptosis because the levels of caspase- 3 and caspase- 7 activation in the colon of Aim $2^{-/-}$mice decreased compared with that in WT mice (Figure 4-4G-H). To examine possible intrinsic defects in the mucus layer in mice lacking AIM2, we analyzed the thickness of the mucus layer and the expression of genes encoding mucin, MUC2, MUC3 and MUC4, in WT and Aim2 ${ }^{-/-}$ mice. We found that the thickness of the mucus layer lining the large intestine and the expression levels of genes encoding MUC2, MUC3 and MUC4 were similar in WT and Aim $2^{-1-}$ mice (Figure 4-5A-B). In addition, we quantified the number of goblet cells and observed similar numbers of these cells in the crypts of WT and $\mathrm{Aim} 2^{-/-}$mice

(Figure 4-5C).

\section{AIM2 Controls Expansion of Intestinal Stem Cells}

We and others have previously identified that intestinal stem cells as the "cell-oforigin" of intestinal cancer [77, 132]. We hypothesized that the dramatic increase in cell proliferation and tumor formation in $\mathrm{Aim}^{-/-}$mice was caused by a population of intestinal stem cells which is susceptible to oncogenic insults. To investigate this, we cultivated and measured the growth rate of stem cells harvested from the colonic epithelia of untreated WT and Aim $2^{-/-}$mice. Remarkably, colonic epithelial stem cells harvested from $\mathrm{Aim}^{-/-}$mice consistently formed more organoids over five days compared with those harvested from WT mice (Figure 4-6). In addition, we measured the diameters of these organoids and found a greater proportion of organoids originated from $\mathrm{Aim}^{-/-}$mice with a larger size compared with those isolated from WT mice (Figure 4-6). These data indicated that stem cells derived from the colon of $\mathrm{Aim}^{-/-}$mice exhibited an increased capacity to proliferate.

We and others have previously demonstrated that stem cells or progenitor cells in the small intestine of mice are highly susceptible to tumorigenesis induced by aberrant activating $\beta$-catenin mutations $[77,133]$. These cells are marked by Prom1 (CD133), a marker of cancer stem cells in human intestinal tumors that arise as a result of aberrant Wnt signaling $[134,135]$. To investigate the role of AIM2 in this pathway, we used the Prom $1^{\text {CreERT2-LacZ }(C-L) /+}$; Ctnnb1 ${ }^{\text {lox(ex } 3) /+}$; RosaZsGreen mouse strain to investigate proliferation of Prom $1^{+}$cells following aberrant Wnt signaling [77]. The Prom $1^{C-L /+}$ mouse strain contains an inducible Cre and a nuclear LacZ reporter allele knocked into the Prom 1 locus, which allowed us to detect cells expressing Prom 1 using $\beta$ galactosidase staining. This mouse strain also encodes a Cre-dependent RosaZsGreen reporter allele for use in lineage tracing, which is expressed irreversibly in Prom $1^{+}$cells when CreER ${ }^{\mathrm{T} 2}$ is induced from the Prom $1^{C-L /+}$ locus following tamoxifen treatment. Further, the Wnt signaling pathway is aberrant in this mouse strain owing to the presence of a Cre-dependent mutant allele of $\beta$-catenin $\left(C_{t n n b} 1^{\text {lox(ex3) }}\right)$. To determine whether colon cells from mice lacking AIM2 exhibited increased tumor susceptibility to Wnt signaling, we crossed Aim $2^{-/-}$mice to Prom $1^{\mathrm{C}-L /+} ; \mathrm{Ctnnbl}^{\text {lox(ex3)/+}} ;$ RosaZsGreen mice. 


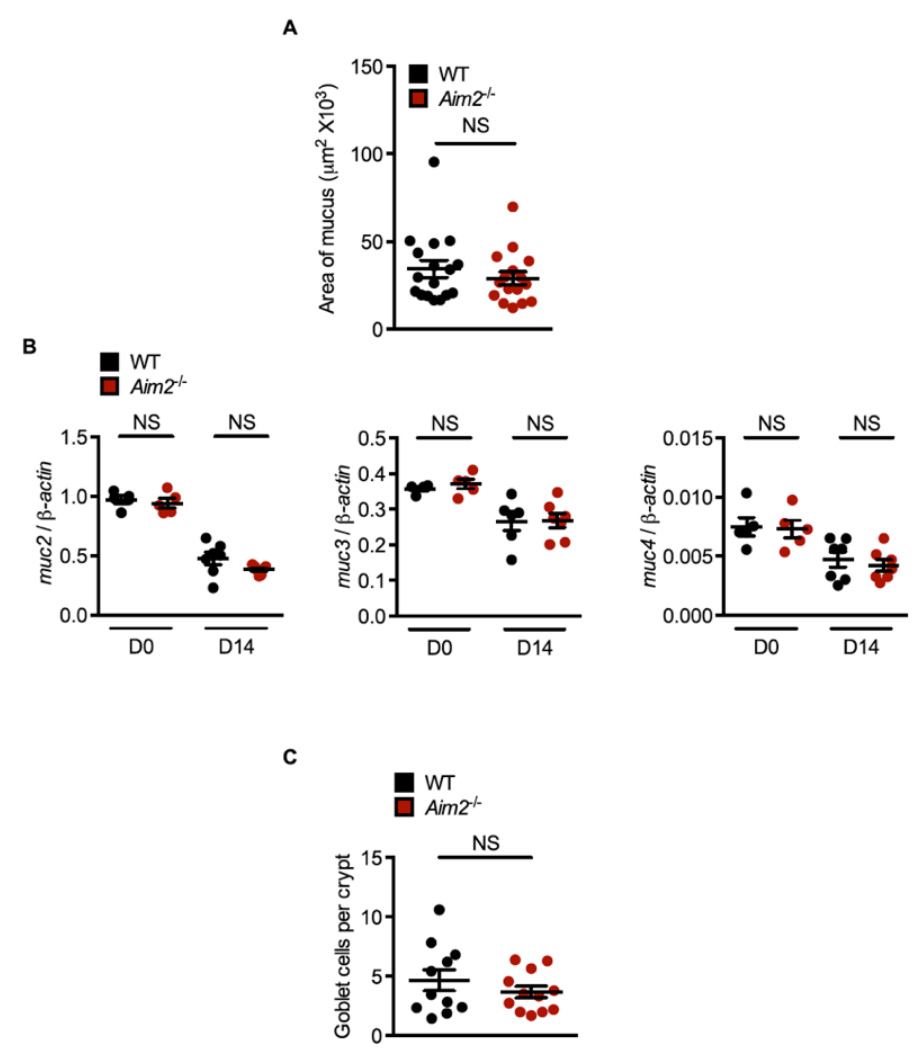

Figure 4-5. Intact mucus layer in WT and $\operatorname{Aim}^{-/-}$mice.

(A) Area of the mucus layer was measured from 5-7 different sections of the same mouse using ImageJ ( $\mathrm{n}=3$ mice). (B) Relative expression of genes encoding MUC2, MUC3, and MUC4 in untreated WT and Aim2 ${ }^{-/-}$mice or in WT and Aim2 $2^{-/-}$mice 14 days after injection of AOM. (C) The number of goblet cells was quantified in each crypt (50 crypts per mouse) in the colon of WT and Aim2/ mice stained with PAS and Alcian blue. Two tailed $t$ test $(\mathrm{A}-\mathrm{C})$. NS, not statistically significant. Data represent one experiment (mean and SEM). 

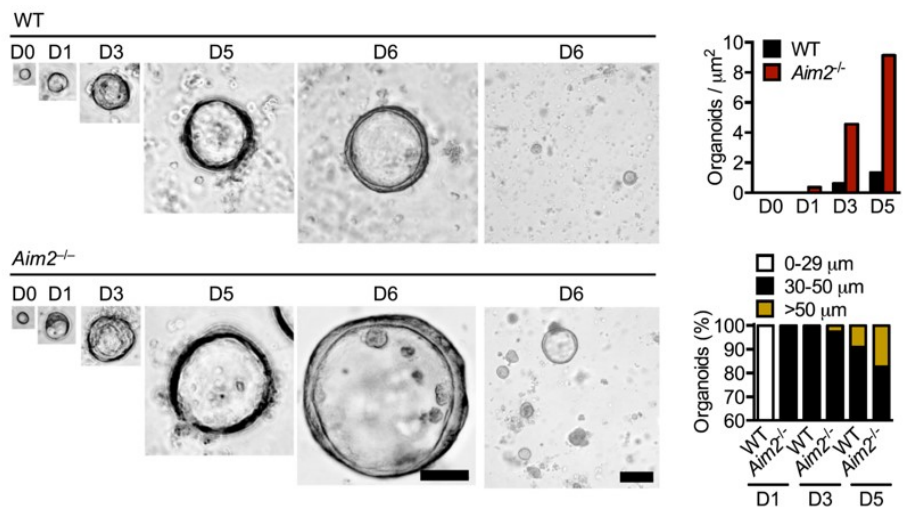

Figure 4-6. AIM2 controls proliferation of intestinal progenitor stem cells.

Formation of organoids derived from colonic stem cells harvested from untreated WT and Aim $2^{-1-}$ mice. Quantification of the number (top right) and size of organoids (bottom right). Scale bars, $50 \mathrm{~mm}$ (left) or $200 \mathrm{~mm}$ (right). Data represent one of three independent experiments. 
We used $\beta$-galactosidase staining to detect nuclear LacZ expression from the Prom 1 promoter. We found that a loss of AIM2 did not alter Prom1 expression pattern in the large intestine and the majority of cells in the colonic crypts expressed Prom1

(Figure 4-7A). Remarkably, three weeks after induction of aberrant $\beta$-catenin activation by tamoxifen treatment, we observed a significant increase in the stem cell activity of Prom $1^{+}$cells, indicated by GFP lineage tracing using the RosaZs $G$ allele, in the colon of Prom $^{\mathrm{C}-\mathrm{L} /+}$; Ctnnb1 ${ }^{\text {lox(ex3)/+}}$; RosaZsG; Aim2 $2^{-/-}$mice compared with Prom $1^{\mathrm{C}-\mathrm{L} /+}$; Ctnnb $1^{\text {lox(ex3)/+}}$; RosaZsG; Aim $2^{+/+}$mice (Figure 4-8A). There was a significantly increased number of $\mathrm{GFP}^{+}$cells in Prom $\mathrm{I}^{\mathrm{C}-\mathrm{L} /+} ; \mathrm{Ctnnbl}^{\mathrm{lox}(\mathrm{ex} 3) /+} ; \operatorname{RosaZsG}$; Aim2 $2^{-{ }_{-}}$mice

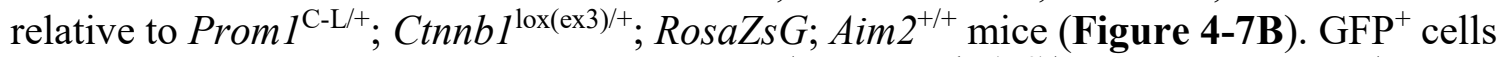
populated almost the entire crypts in Prom $^{\mathrm{C}-\mathrm{L} /+} ;$ Ctnnbl $^{\operatorname{lox}(\mathrm{ex} 3) /+} ; \operatorname{RosaZsG} ;$ Aim2 $^{-/-}$mice (Figure 4-8A). We observed very few crypts that were comprised of $\mathrm{GFP}^{+}$cells in Prom $^{\mathrm{C}-\mathrm{L} /+} ;$ Ctnnb1 $^{\mathrm{lox}(\mathrm{ex} 3) /+} ; \operatorname{RosaZs} G ;$ Aim $^{+/+}$mice (Figure 4-8A), indicating that a small population of Prom $1^{+}$cells could still potentially behave as stem or progenitor cells in the colon of $\mathrm{Aim}^{+/+}$mice. These findings suggested that the lack of AIM2 increased the stem cell activity of Prom $1^{+}$cells following aberrant Wnt activation.

Prom $1^{C-L /+} ;$ Ctnnbl $1^{\text {lox(ex3)/+}} ;$ RosaZs $G$ mice succumb within six weeks of tamoxifen induction owing to extensive tumor formation initiated from Prom $1^{+}$stem cells in the small intestine (Figure 4-8B). Although we did not observe macroscopic tumors in the large intestine of these animals, we found an elevated number of $\mathrm{Ki}^{+} 7^{+}$cells, increased staining for phosphorylated AKT, total AKT and c-Myc and a small number of

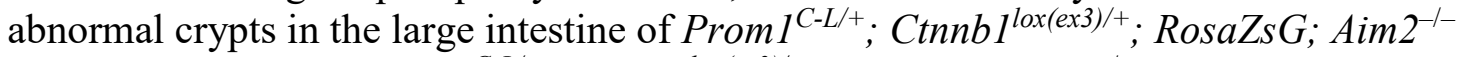

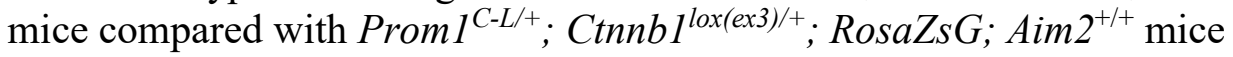

(Figure 4-7C and Figure 4-8A). We also noticed accelerated tumorigenesis, increased staining for phosphorylated AKT and c-Myc in the small intestine of $\operatorname{Prom} 1^{C-L /+}$; Ctnnb1 ${ }^{\text {lox }(e \times 3) /+}$; RosaZsG; Aim2 $2^{-/-}$mice compared with Prom $1^{C-L /+} ;$ Ctnnb1 $^{\text {lox }(e x 3) /+}$; RosaZsG; Aim $^{+/+}$mice (Figure 4-8B). These results validated the colitis-associated colorectal cancer model and suggested that AIM2 played a role in protecting tumor formation in the intestine. Overall, loss of AIM2 resulted in an increased stem cell activity in the colon following aberrant Wnt-signaling in the intestinal stem-cell niche.

To further confirm a role for the epithelial compartment in AIM2-mediated protection against colon cancer development, we performed bone marrow transplantation experiments to examine the contribution of the radioresistant stromal compartment relative to that of the hematopoietic compartment in governing colon tumorigenesis. As expected, Aim $^{-1-}$ mice that received $\mathrm{Aim}^{-/-}$bone marrow were more susceptible to tumorigenesis compared to WT mice that received WT bone marrow (Figure 4-9A). After 80 days post-AOM injection, Aim $2^{-/-}$mice that received WT bone marrow had significantly increased tumor burden compared to WT mice that received WT bone marrow (Figure 4-9B-C). These results provided evidence to suggest that AIM2 in the stromal compartment could play a role in the protection against colorectal tumorigenesis. WT mice that received im $^{-/-}$bone marrow also showed slightly increased tumor burden compared with WT mice that received WT bone marrow, suggesting that the bone marrow compartment contributed to colorectal tumorigenesis via a yet-undefined mechanism. 
A
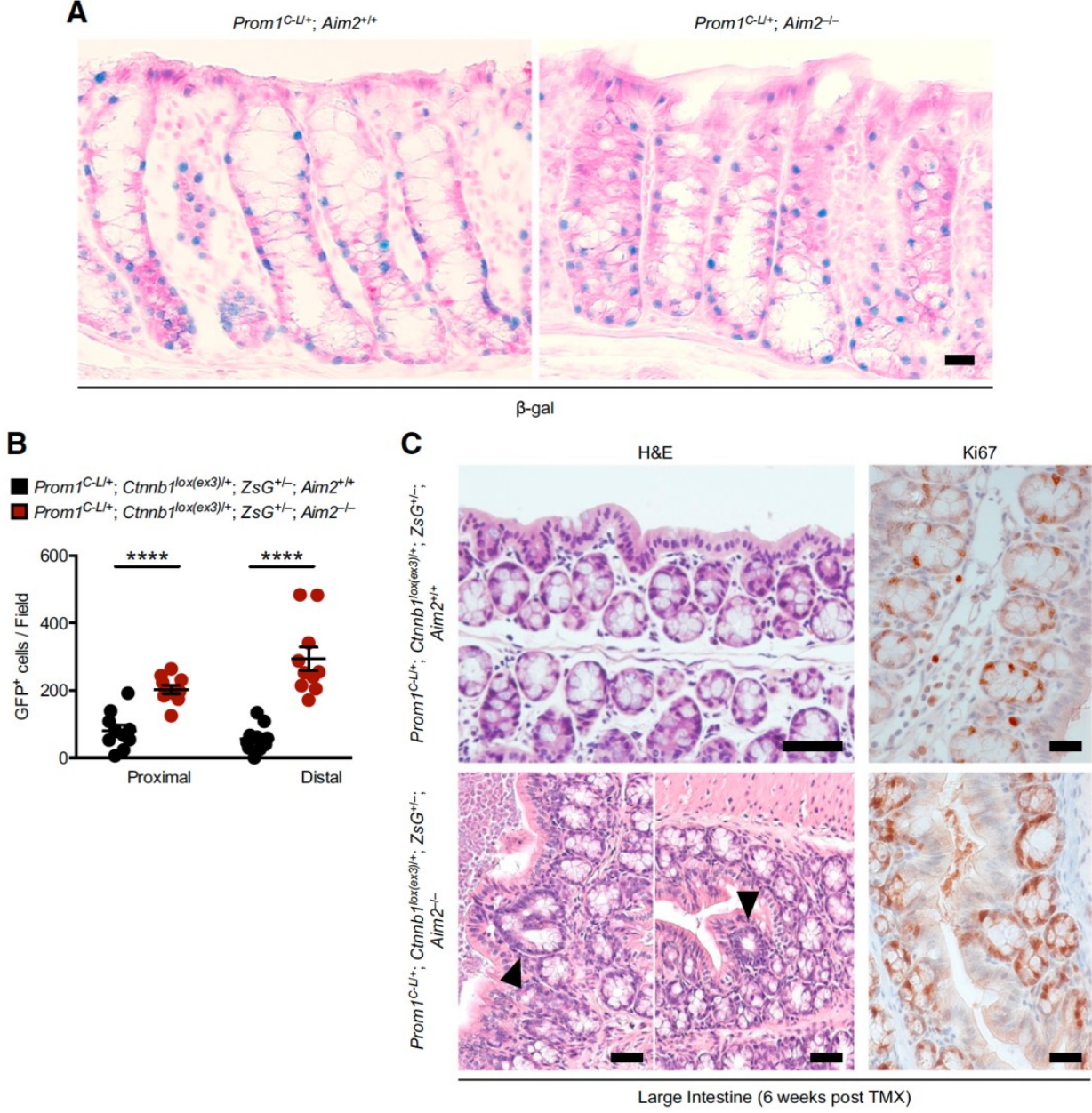

Figure 4-7. Loss of AIM2 increased proliferation initiated by Prom1 ${ }^{+}$cells in the large intestine following $\boldsymbol{\beta}$-catenin activation.

(A) b-galactosidase staining of Prom1-expressing cells indicating similar expression pattern in the large intestine of Prom $1^{\mathrm{C}-\mathrm{L} /+} ;$ Aim $^{+/+}$and Prom $1^{\mathrm{C}-\mathrm{L} /+} ;$ Aim $^{-/-}$mice. Scale bar, $20 \mathrm{~mm}$. (B) Quantification of the number of $\mathrm{GFP}^{+}$cells per field in the large intestine of Prom $1^{\mathrm{C}-\mathrm{L} /+} ;$ Ctnnbl $^{\text {lox(ex3)/+}} ; \operatorname{RosaZsG} ;$ Aim2 $^{+/+}$and Prom $1^{\mathrm{C}-\mathrm{L} /+} ;$ Ctnnbl $^{\text {lox }(\mathrm{ex} 3) /+}$; RosaZsG; $\mathrm{Aim}^{-1-}$ mice 3 weeks post-tamoxifen (TMX) induction. Fields from 10 images for each genotype were quantified. (C) H\&E (left) and Ki67 (right) staining of the large

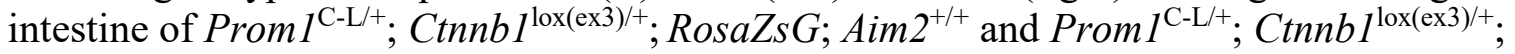
RosaZsG; Aim $2^{--}$mice 6 weeks post-TMX induction. Arrowheads indicate abnormal crypts. Scale bar, $50 \mathrm{~mm}$ (H\&E, top), $100 \mathrm{~mm}$ (H\&E, bottom), $200 \mathrm{~mm}$ (Ki67). Mean and SEM in (B). $* * * * p<0.0001$ (two-tailed t test [B]). 

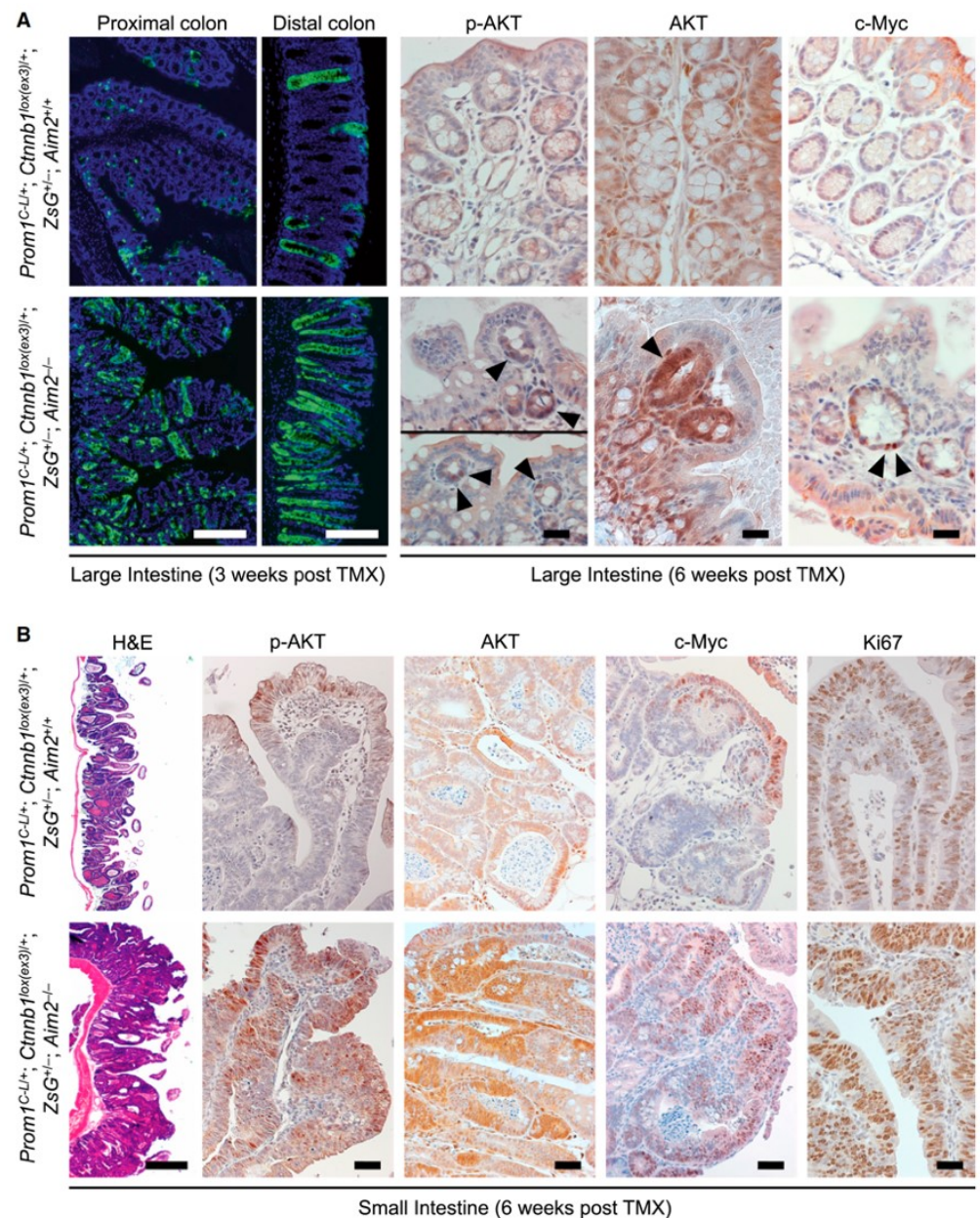

Figure 4-8. AIM2 controls expansion of intestinal stem cells.

(A) Lineage tracing (left) of Prom $1^{+}$cells (green) in the large intestine 3 weeks posttamoxifen (TMX) induction. Blue staining indicates DAPI. Scale bar, $100 \mathrm{~mm}$. Staining for phosphorylated AKT (p-AKT, Ser473), total AKT and c-Myc (right) in the large intestine 6 weeks post-TMX induction. Scale bar, $200 \mathrm{~mm}$. Arrow heads indicate early lesions with intense staining for the respective markers. (B) H\&E staining and staining for p-AKT (Ser473), total AKT, c-Myc, and Ki67 staining in the small intestine 6 weeks post-TMX induction. Scale bars, $250 \mathrm{~mm}(\mathrm{H} \& \mathrm{E})$ and $100 \mathrm{~mm}$ (p-AKT, total AKT, cMyc, and Ki67 staining). 

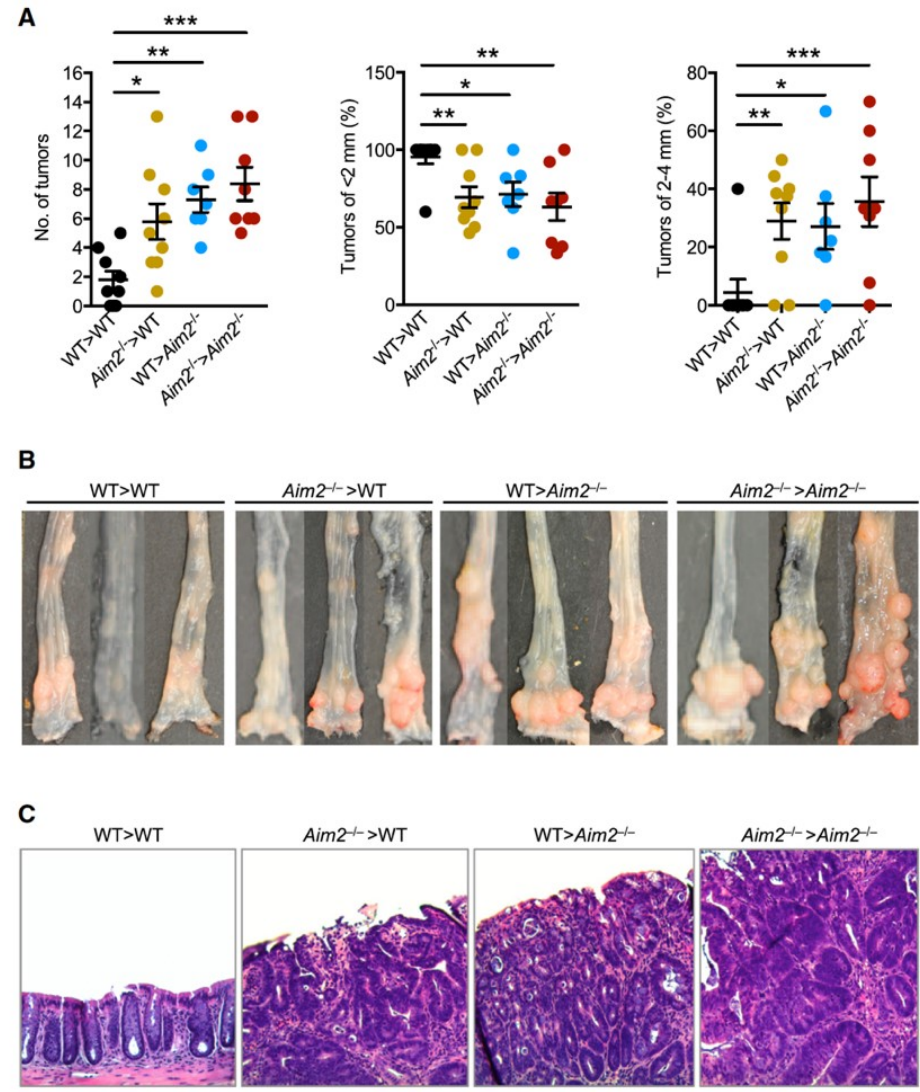

Figure 4-9. The role of hematopoietic and non-hematopoietic compartments in AIM2-mediated protection of colorectal tumorigenesis.

(A) Four groups of mice were generated by bone marrow transplantation: (1) WT $\rightarrow$ WT, n =9; (2) Aim $2^{-/-} \rightarrow \mathrm{WT}, \mathrm{n}=9$; (3) WT $\rightarrow \operatorname{Aim}^{-/-}, \mathrm{n}=7$; (4) $\operatorname{Aim}^{-/-} \rightarrow \operatorname{Aim}^{-/-}, \mathrm{n}=8.6$ weeks following bone marrow transplantation, mice were treated with AOM followed by DSS, and colon tumors were counted 80 days after injection of AOM. The numbers of tumors in the colon and percentages of tumors of less than $2 \mathrm{~mm}$ or $2-4 \mathrm{~mm}$ in diameter were quantified. (B) Representative images of colon tumors. (C) Representative H\&E images of colon tumors. Magnification: $20 \times$. Data represent one experiment (mean and SEM). ${ }^{*} \mathrm{p}<0.05 ; * * \mathrm{p}<0.01 ; * * * \mathrm{p}<0.001$. One-way ANOVA (A). 


\section{AIM2 Protects Colorectal Tumorigenesis by Modulating the Gut Microbiota}

Intestinal cell proliferation and the development and progression of colorectal cancer is regulated by the composition of gut microbiota. Studies have reported that the NOD-like receptor protein NLRP6 modulates the microbial ecology and protects against colon tumor development $[136,137]$. To assess whether the gut microbiota plays a role in the increased tumorigenic susceptibility of $\mathrm{Aim} 2^{-/}$mice, we performed $16 \mathrm{~S}$ rRNA gene sequencing analysis of the gut microbiota derived from stool samples of separately housed WT and im $^{-/-}$mice. Our 16S rRNA gene sequencing analyses revealed a markedly distinct intestinal microbial landscape in separately housed WT and $\mathrm{Aim}^{-1-}$ mice (Figure 4-10A-B). Relative to WT mice, Aim $2^{-/-}$mice harbored increased levels of Akkermansia muciniphila, Anaeroplasma, and decreased levels of Anaerostipes, Bifidobacterium, Flexispira, Prevotella and Paraprevotella species (Figure 4-10A). Of these, previous reports have linked an increase in Akkermansia and a decrease in Prevotellaceae with the development of colonic tumorigenesis [138]. Interestingly, cohousing equilibrated the relative abundance of Anaerostipes, Flexispira, Prevotella, and Paraprevotella in WT and Aim $2^{-/-}$mice (Figure 4-10A-B).

We took advantage of the transmissible nature of the gut microbiota to investigate whether susceptibility to colorectal tumorigenesis could be reduced in $\mathrm{Aim} 2^{-/-}$mice by co-housing them with WT mice. We found a striking reduction in the number and incidence of colon tumors in co-housed $\mathrm{Aim}^{-/-}$mice compared with separately housed Aim $2^{-/-}$mice (Figure 4-10C-D). Intriguingly, WT mice co-housed with $\mathrm{Aim} 2^{-/-}$mice responded conversely and developed more tumors compared with their separately housed controls (Figure 4-10C-D). Co-housed $\mathrm{Aim}^{-/-}$mice were still more susceptible to tumorigenesis compared with co-housed WT mice (Figure 4-10C-D), supporting the concept that both genetic and environmental factors contributed to the development of cancer.

\section{Discussion}

Members of the pattern-recognition receptor family are central players in the regulation of infectious, inflammatory and metabolic diseases. Our laboratory and others have previously shown that the innate immune receptors NLRP3, NLRP6, NLRP12, NLRC4, STING and NOD2 are required for the protection against colitis and colitisassociated tumorigenesis [27-31, 139-143]. However, the role of AIM2-like receptors, including AIM2 and IFI16 or p202, in regulating gastrointestinal health and disease is unknown.

The innate immune receptor AIM2 was originally isolated from human melanoma cells [37]. AIM2 directly binds dsDNA and initiates the recruitment of ASC and caspase1 to assemble a multi-protein inflammasome complex. Whether AIM2 can operate independently of inflammasomes in the cell is unclear. Here, we identified an unexpected function for AIM2 that departs from its known role in inflammasome signaling. Certain members of the NLR family, including NLRP6 and NLRP12, have also been shown to 


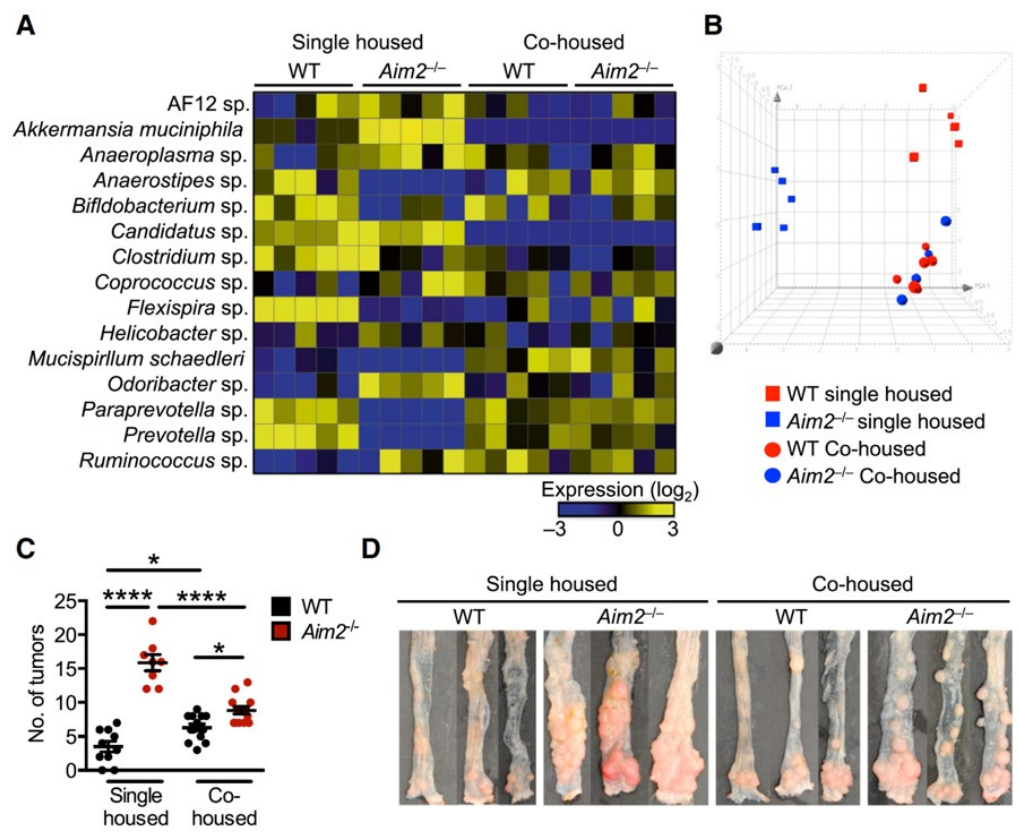

Figure 4-10. Reciprocal exchange of gut microbiota reduces susceptibility of $\operatorname{Aim}^{-}$ - mice to colon cancer.

(A) Bacterial species from feces that had the greatest difference in abundance between single housed WT and Aim $2^{-/-}$mice and their relative abundance in co-housed WT and Aim $2^{-1-}$ mice, based on analysis of the $16 \mathrm{~S}$ rRNA gene, are shown in a heatmap in alphabetical order. (B) Principal component analysis of (A). (C) The total number of tumors observed in the colon of separately housed and co-housed mice 80 days after injection of AOM. (D) Representative images of colons in (C). Data represent one experiment (A and B) or pooled from two independent experiments (C and D) (mean and $\mathrm{SEM}$ in $[\mathrm{C}]) .{ }^{*} \mathrm{p}<0.05 ; * * * * \mathrm{p}<0.0001$. One-way ANOVA (C). 
exert inflammasome-dependent and inflammasome-independent functions in health and disease [144].

The mechanism driving AIM2-dependent protection against tumorigenesis is the ability of AIM2 to tightly suppress overt cellular proliferation of intestinal epithelial cells. Whether AIM2 directly interacts and prevents phosphorylation of the kinase AKT is unclear. Unlike the role for NLRP6 in targeting goblet cell mucin secretion [145], AIM2 specifically controlled expansion and proliferation of intestinal stem cells. The larger population of cells derived from Prom $1^{+}$cells lining the intestinal crypt in mice lacking AIM2 following aberrant induction of $\beta$-catenin indicated that these cells might be highly proliferative and less well-differentiated. In vitro studies have shown that ectopic expression of AIM2 can be used to promote cell cycle arrest and inhibition of cell proliferation [146]. These findings, together, highlight the dynamic roles of AIM2 in regulating cell functions during cell growth and tumorigenesis.

In mice exposed to AOM-DSS, the expression of AIM2 was down-regulated as early as 14 days, suggesting that robust AIM2 expression was critical for preventing early tumorigenic events. The levels of AIM2 expression in tumor tissues of patients have been shown to greatly influence the outcome of the disease. Patients who have tumor cells that completely lacked AIM2 expression have a more than 3-fold higher mortality rate than patients whose tumor cells retained some AIM2 expression [26]. The precise molecular signal that triggers down-regulation of AIM2 expression during colorectal tumorigenesis is unknown and therapeutic strategies aimed at increasing colonic AIM2 expression may help to prevent overt tumorigenesis in susceptible individuals.

Of particular clinical relevance is the observation that defective AIM2 function leading to tumorigenesis can be partially prevented by a transmissible gut microbiota in cohoused mice. This finding indicates that engraftment of healthy donor gut microbiota to patients suffering chronic intestinal inflammation could reduce the risk of developing colorectal cancer. The microbiota plays a central role in modulating cell proliferation in the intestine. Gut-associated Lactobacillus murinus enhances cell proliferation in the mouse intestine [147]. Furthermore, gut microbiota has the capacity to induce IL-17C production in intestinal epithelial cells via a MyD88-dependent pathway, which leads to increased expression of the prosurvival proteins Bcl2 and Bcl- $\mathrm{x}_{\mathrm{L}}$ to drive colorectal tumorigenesis [148]. Carbohydrate-derived metabolites generate by gut microbiota has also been shown to enhance colon epithelial cell proliferation in an $A p c^{\mathrm{Min} /+}$ mouse model lacking the gene encoding the DNA mismatch repair protein MutS homolog 2 (MSH2) [149].

During barrier damage, it is possible that DNA from microbial species that have invaded intestinal cells or DNA from dying host cells could be sensed by AIM2 in intestinal cells. It is tempting to hypothesize that instead of contributing to the inflammatory response further by inducing activation of the inflammasome, AIM2 responds by dampening cellular proliferation in the intestine. How AIM2 might be sensing different environmental cues in the cytoplasm to direct context-specific cellular processes is an exciting question for future investigation. In conclusion, our findings 
demonstrated a requirement for AIM2 in the protection against colorectal cancer. Therapeutic modulation of AIM2 expression and gut microbiota could play a central role in reducing the risk of developing colorectal cancer. 


\section{CHAPTER 5. DETRIMENTAL TYPE I INTERFERON SIGNALING DOMINATES PROTECTIVE AIM2 INFLAMMASOME RESPONSES DURING FRANCISELLA NOVICIDA INFECTION*}

\section{Introduction}

Innate immune sensors mediate the recognition of PAMPs and DAMPs and are central regulators of host defense against microbial infections. Upon activation, immune sensors induce the production of inflammatory cytokines and interferons. Certain innate immune cytoplasmic sensors have a unique ability to form a caspase-1-activating multiprotein complex termed the inflammasome. These sensors include members of the nucleotidebinding oligomerization domain (NOD), leucine-rich repeat (LRR)-containing protein family members, AIM2 and pyrin [150]. Of these, AIM2 binds directly to dsDNA and assembles the inflammasome to mediate the release of interleukin IL- $1 \beta$ and IL-18 and the induction of pyroptosis. AIM2 orchestrates protective inflammasome-dependent responses against microbial infection in vivo, including infection by the Gram-negative bacterial pathogen $F$. novicida [150].

Components of the type I IFN signaling pathway are essential for the activation of AIM2 inflammasome during infection by $F$. novicida and L. monocytogenes $[41-45,151$, 152]. During $F$. novicida infection, the DNA sensor, cGAS, and its adaptor, STING, mediate the production of type I IFNs, which in turn drive expression of effector proteins of cell-autonomous immunity leading to bacteriolysis and release of bacterial DNA for recognition by AIM2 [150].

Type I IFN signaling during bacterial infection in vivo can be beneficial or detrimental depending on the pathogen [153]. Whereas type I IFN signaling is protective in mice infected with Streptococcus pneumoniae, or Streptococcus pyogenes, it is detrimental in case of $F$. novicida or L. monocytogenes [50]. The requirement for type I IFN signaling to activate the AIM2 inflammasome in macrophages is counterintuitive to the observation that, type I IFN signaling is detrimental during in vivo infection with $F$. novicida while the AIM2 inflammasome is protective. How the interplay between the opposing effects of these signaling pathways determines the final outcome during $F$. novicida infection has remained unclear. Here, we showed that the detrimental effects of type I IFN signaling overrides protective AIM2 inflammasome responses.

\footnotetext{
* Adapted with open access permission. Zhu, Q., Man S.M., Karki R., Malireddi R.K., Kanneganti, T.D. (2018). Detrimental type I Interferon signaling dominates protective AIM2 Inflammasome responses during Francisella novicida Infection. Cell Reports. 22, 3168-3174.
} 


\section{Results and Discussion}

\section{IFNAR Signaling Enhances Susceptibility to Infection by F. novicida}

A recent study has demonstrated non-redundant functions for type I IFN receptor subunits IFNAR1 and IFNAR2 independently of one another [154]. To directly compare the effects of IFNAR1 and IFNAR2 during $F$. novicida infection, we infected WT mice and mice lacking IFNAR1 (Ifnar $1^{--}$) or IFNAR2 (Ifnar ${ }^{-/-}$) with $F$. novicida and monitored their survival. Whereas $65 \%$ of the infected WT mice died within 14 days, only $13 \%$ of the Ifnar $1^{-/-}$mice and $11 \%$ of the Ifnar $2^{-/}$mice succumbed to infection (Figure 5-1A), suggesting that signaling by both IFNAR1 and IFNAR2 contribute to increased susceptibility to infection by $F$. novicida. The resistance to $F$. novicida infection in Ifnar $1^{-/-}$mice is consistent with the observations of a previous study [155]. Furthermore, only $8 \%$ of mice lacking the transcription factor IRF9 $\left(\operatorname{Irf} 9^{-/-}\right)$, a component of ISGF3 transducing type I IFN signaling, succumbed to infection by $F$. novicida over 14 days, essentially phenocopying Ifnar $1^{-/-}$and Ifnar $2^{-/-}$mice (Figure 5-1A). IRFs are central components regulating type I IFN production in response to diverse pathogens. Indeed, we found that $\operatorname{Irf} 7^{-/}$and $\operatorname{Irf} 3^{-1-} \operatorname{Irf} 7^{-/-}$mice were resistant to the infection (Figure 5-1B). However, mice lacking IRF5, an IRF not critical for IFN production, $\left(\operatorname{Irf5} 5^{--}\right)$were as susceptible as WT mice (Figure 5-1B). We also observed increased resistance in Sting ${ }^{\mathrm{gt} / \mathrm{gt}}$ mice (Figure 5-1B). Consistent with these results, we found significantly lower bacterial burdens in the liver and spleen of Sting ${ }^{\mathrm{gt} / \mathrm{gt}}$ mice, $\operatorname{Irf} 7^{-/}$mice, and $\operatorname{Irf} 9^{-/-}$mice 3 days after infection compared with WT mice (Figure 5-1C). Together, these results suggest that the effector responses mediated by type I IFN signaling enhance susceptibility to $F$. novicida infection in vivo.

\section{The Detrimental Effects of Type I IFN Signaling Dominate the Protective Effects of AIM2 during $F$. novicida Infection in vivo}

To directly assess the relative contribution of IFNs and AIM2 in host defense during $F$. novicida infection, we compared mice lacking components of the type I IFN signaling pathway, such as IFNAR2, with mice lacking AIM2. Whereas Ifnar $2^{-/-}$mice were resistant to infection by $F$. novicida, with only $19 \%$ of the mice dying, all $\mathrm{Aim}^{-/}$ mice succumbed to infection within 6 days (Figure 5-2A). The disparate roles of type I IFN signaling and AIM2 during $F$. novicida infection in vivo suggest a more complex functional relationship between these two pathways.

To formally investigate the cumulative effects of type I IFN signaling and AIM2 during $F$. novicida infection, we bred Ifnar $2^{-1-}$ mice and $A$ im $2^{-1-}$ mice to generate mice

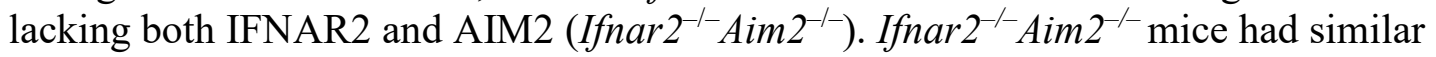
numbers of neutrophils, macrophages, dendritic cells, $\mathrm{CD} 8^{+} \mathrm{T}$ cells and $\mathrm{B}$ cells in the spleen, $\mathrm{T}$ cells in the thymus, and neutrophils, monocytes and B cells in the bone marrow compared with WT mice (Figure 5-3A-C). We infected Ifnar $2^{-1-}$ Aim $2^{-/}$mice with $F$. novicida and monitored their survival. Interesting, Ifnar $2^{--}$Aim $2^{-/-}$mice were resistant to 

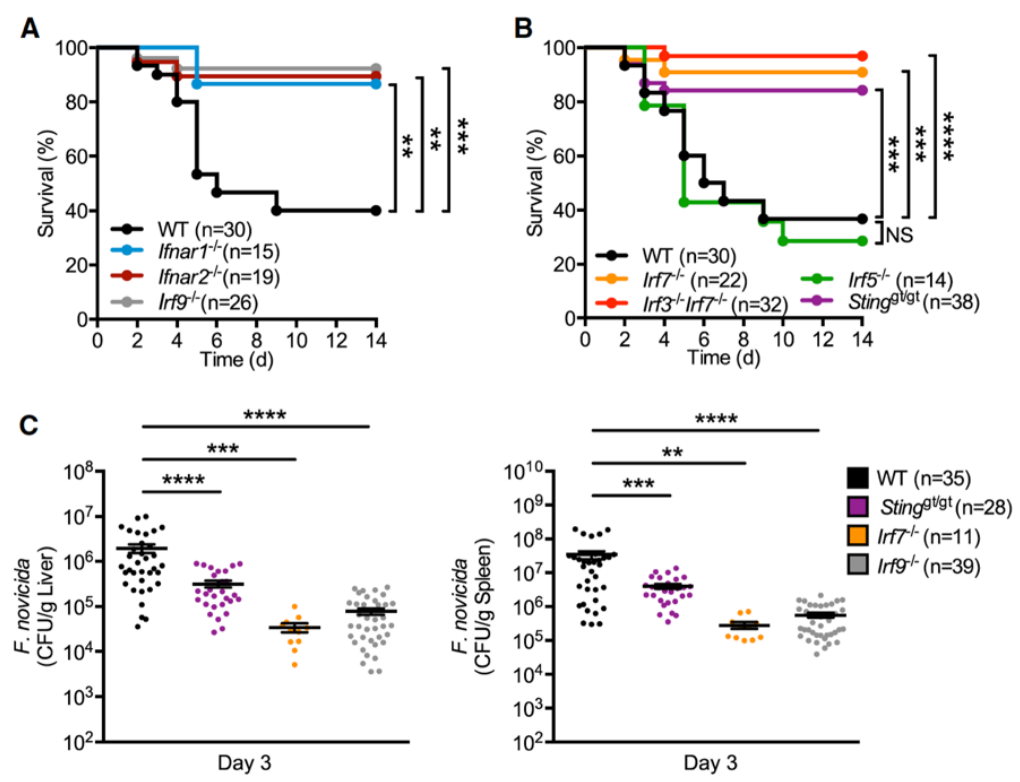

Figure 5-1. Mice lacking IFNAR1 or IFNAR2 are resistant to $\boldsymbol{F}$. novicida.

(A) Survival of WT, Ifnarl ${ }^{-/-}$, Ifnar2 $2^{-/-}$, and $\operatorname{IrfS}^{-/-}$mice infected with $F$. novicida. (B) Survival of WT, Irf7 ${ }^{-/}, \operatorname{Irf} 3^{-/-} \operatorname{Irf7}^{--}, \operatorname{Irf5} 5^{-/}$and Sting ${ }^{\text {gt/gt }}$ mice infected with F. novicida. (C) Bacterial burdens in the liver and spleen of WT, Sting ${ }^{\mathrm{gt} / \mathrm{gt}}, \operatorname{Irf7/}$, and $\operatorname{IrfS}^{-/-}$mice 3 days after infection with $F$. novicida. NS, not significant; **p $<0.01, * * * \mathrm{p}<0.001$, $* * * * \mathrm{p}<0.0001$; log-rank test (A and B) or one-way ANOVA with Dunnett's multiplecomparisons test $(\mathrm{C})$. Data are pooled from three $(\mathrm{C})$ or four (A and B) independent experiments (mean and SEM in $\mathrm{C}$ ). 

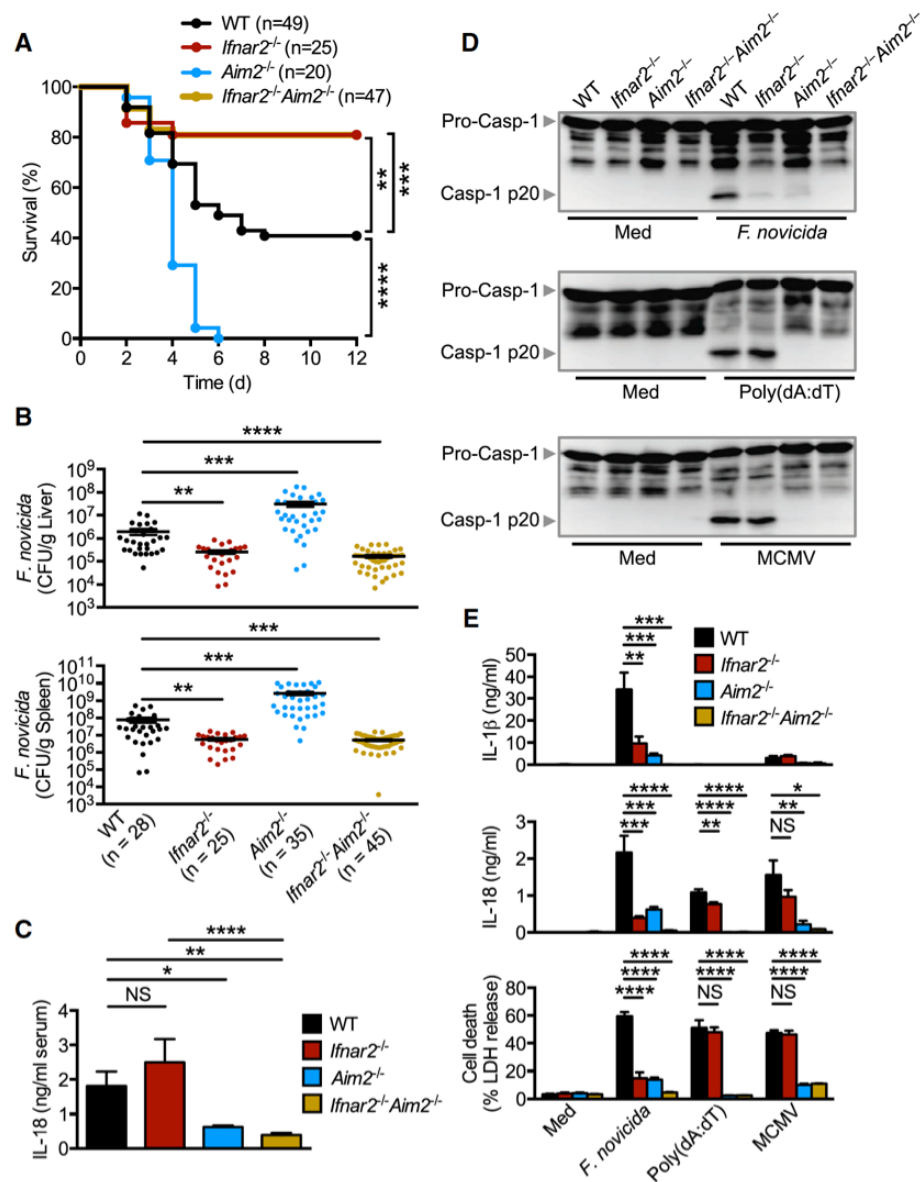

Figure 5-2. Detrimental role of type I IFN signaling is dominant over AIM2 inflammasome responses during $F$. novicida infection.

(A) Survival of WT, Ifnar2 $2^{--}$, Aim $2^{-/-}$, and Ifnar $2^{-1-}$ Aim $2^{-/-}$mice infected with $F$. novicida. (B) Bacterial burdens in the liver and spleen of WT, Ifnar $2^{-/-}$, Aim $2^{-1-}$, and Ifnar $2^{-/}$Aim $2^{-/-}$mice 3 days after $F$. novicida infection. (C) Concentration of IL-18 in the sera of WT, Ifnar $2^{-/-}$, Aim $^{-/-}$, and Ifnar $2^{-/-}$Aim $2^{-/-}$mice 1 day after infection. (D) Immunoblot analysis of pro-caspase-1 (Pro-Casp-1) and the caspase-1 subunit p20 (Casp1p20) in unprimed WT or mutant BMDMs left uninfected or untreated (Med, medium alone) or assessed 20 hours after infection with $F$. novicida (MOI, 100; top), 5 hours after transfection with poly(dA:dT) (middle), or 10 hours after infection with MCMV (bottom). (E) Release of IL-1b (top) or IL-18 (middle) and death (bottom) of unprimed BMDMs after treatment, as in (D). NS, not significant; *p $<0.05 ; * * p<0.01, * * * p<$ $0.001, * * * * p<0.0001$; long-rank test (A) or one-way ANOVA with Dunnett's multiple comparisons test (B, C, and E). Data are pooled from three (B) or four (A and C) independent experiments or are from one experiment representative of two independent experiments (D and E) (mean and SEM in B, C, and E). 
A
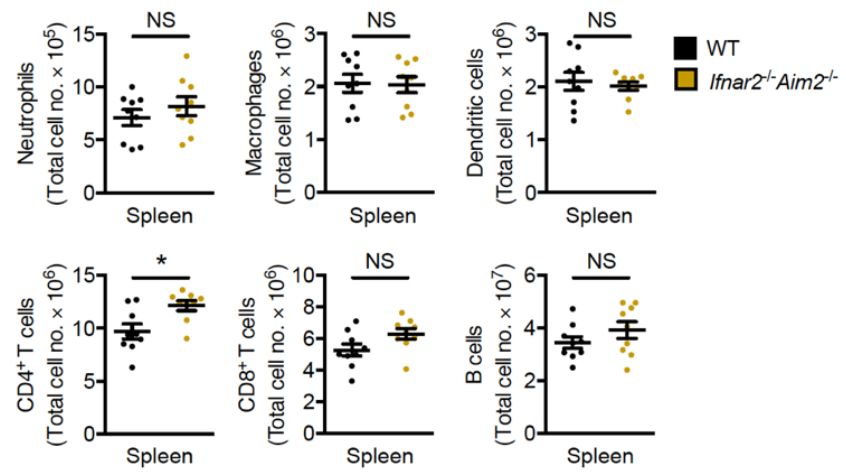

B

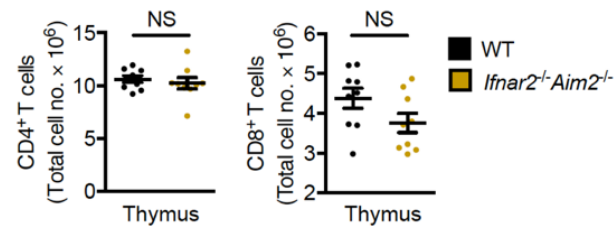

C

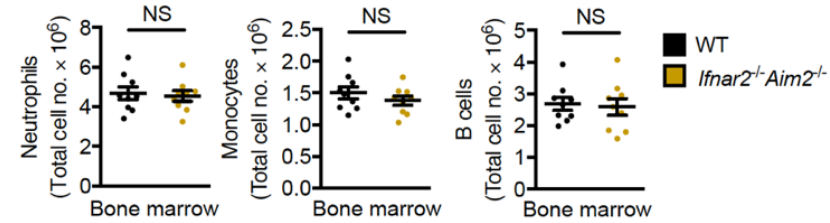

Figure 5-3. Characterization of Ifnar2 ${ }^{-1-} \operatorname{Aim} 2^{-/-}$mice.

(A) Total number of neutrophils, macrophages, $\mathrm{DCs}, \mathrm{CD}^{+} \mathrm{T}$ cells, $\mathrm{CD} 8^{+} \mathrm{T}$ cells, and $\mathrm{B}$ cells in the spleen of WT mice and Ifnar $2^{-1-}$ Aim $^{-/-}$mice. (B) Total number of CD4 ${ }^{+} \mathrm{T}$ cells and $\mathrm{CD}^{+} \mathrm{T}$ cells in the thymus of WT mice and Ifnar $2^{-1-}$ Aim $^{-1-}$ mice. (C) Total number of neutrophils, monocytes, and B cells in the bone marrow of WT mice and Ifnar $2^{-/-}$Aim $2^{-/-}$mice. NS, not statically significant; ${ }^{*} P<0.05$ (two-tailed t test $[\mathrm{A}-\mathrm{C}]$ ). Data are pooled from two independent experiments (A-C; mean and SEM in A-C). 
the infection similar to Ifnar $2^{-/-}$mice, with only $19 \%$ of the mice dying of infection over 12 days compared with $100 \%$ in Aim $^{-/-}$mice (Figure 5-2A). Consistent with the reduction in mortality, both Ifnar $2^{-/}$Aim $2^{-/-}$and Ifnar $2^{-/}$mice had significantly lower bacterial burdens in the liver and spleen 3 days after infection compared with WT mice, while Aim $^{-1-}$ mice were more susceptible to infection and had more bacterial burden (Figure 5-2B). These results demonstrate that type I IFN signaling is dominant over AIM2 inflammasome during $F$. novicida infection in vivo. Since both IFNAR1 and IFNAR2 are required for mediating detrimental type I IFN signaling, it is likely that Ifnar $\mathrm{I}^{--} \mathrm{Aim}^{-{ }^{--}}$mice are also resistant to the infection phenocopying Ifnar $2^{-/}$Aim $^{-/-}$ mice. Increased number of splenic $\mathrm{CD}^{+} \mathrm{T}$ cells in naïve Ifnar $2^{--} A \mathrm{im} 2^{--}$mice is unlikely to contribute to the susceptibility (Figure 5-3A), as bacterial burden in Ifnar $2^{-1-}$ Aim $^{-{ }^{--}}$ mice was significantly lower than WT mice 3 days after infection when $\mathrm{T}$ cell responses barely occurred.

We next investigated whether type I IFN signaling was required for AIM2 inflammasome-induced IL-18 production in vivo during $F$. novicida infection. Reduced levels of IL-18 were observed in the sera of Aim $^{2^{--}}$and Ifnar $2^{-/}$Aim $2^{-/}$mice confirming AIM2-dependent IL-18 production in the infected mice (Figure 5-2C). Notably, Ifnar $2^{-/}$ mice retained the ability to produce IL-18 after the infection indicating inflammasome activation independently of type I IFNs in vivo (Figure 5-2C). The comparable level of resistance to F. novicida infection in Ifnar $2^{-/-}$mice and Ifnar $2^{-/} \mathrm{Aim}^{-/-}$mice suggest that the dominance of IFNAR signaling-mediated responses over AIM2 is independent of IL18 .

As demonstrated previously [44], Ifnar $2^{-/}$BMDMs had an impaired ability to activate caspase-1, trigger release of IL-1 $\beta$ and IL-18, and induce cell death in response to F. novicida compared to the WT BMDMs (Figure 5-2D-E). Aim2 ${ }^{-/-}$or Ifnar2 ${ }^{-1-}$ Aim2 $2^{-}$ ${ }^{1}$ BMDMs infected with $F$. novicida also failed to activate inflammasome responses, confirming specificity of $F$. novicida infection to the AIM2 inflammasome

(Figure 5-2D-E). Inflammasome responses occurred normally in Ifnar $2^{-1-}$ cells after poly(dA:dT) transfection or MCMV infection (Figure 5-2D-E). Consistent with previous reports $[44,47]$, transfection of poly $(\mathrm{dA}: \mathrm{dT})$ did not induce IL-1 $\beta$ release in WT BMDMs (Figure 5-2E), possibly due to an inability of poly(dA:dT) transfection in mediating IL$1 \beta$ expression. Collectively, these results demonstrate that unlike in macrophages, AIM2 inflammasome activation in vivo is retained even in the absence of type I IFN signaling.

\section{Inflammatory Cytokine Production Is Intact in the Absence of Type I IFN Signaling}

Previous studies showed that type I IFNs suppress production of proinflammatory cytokines to prevent inflammation-associated damage and lethality during $S$. pneumonia infection [156]. To identify whether the levels of pro-inflammatory cytokines are differentially regulated by type I IFN signaling during infection by $F$. novicida, we analyzed a range of cytokines and chemokines 1 day after $F$. novicida infection, when WT, Ifnar $2^{-/-}$, and Ifnar $2^{-/-}$Aim $2^{-/-}$mice had similar bacterial burden (Figure 5-4A). Levels of the pro-inflammatory cytokines GM-CSF, TNF, IL-6, MIP1- $\alpha$, 
IL-17, IL-12 and IFN $\gamma$ and the anti-inflammatory cytokine IL-10 in the liver were comparable between WT, Ifnar $2^{-1}$, and Ifnar $2^{-1-}$ Aim $^{-1-}$ mice (Figure 5-4B). The levels of these proinflammatory cytokines were also similar in the sera of WT, Ifnar $2^{-/}$, and Ifnar $2^{-1-}$ Aim $2^{-1-}$ mice at this time point (Figure 5-4C). We also measured levels of the cytokines 2 days after infection, when bacterial burdens were similar between the animals (Figure 5-5A). Whereas levels of TNF, IL-6, MIP1- $\alpha$, IFN $\gamma$ and IL-10 were similar in the liver (Figure 5-5B), levels of GM-CSF and IL-12 were decreased in both Ifnar $2^{-/}$ and Ifnar $2^{-/-}$Aim $^{-/-}$mice and level of IL-17 was decreased in Ifnar $2^{-/-}$mice

(Figure 5-5B). Interestingly, we found similar levels of TNF, IL-6, MIP1- $\alpha$ and IL-10 but increased levels IL-17 and IL-12 in the sera of Ifnar $2^{-/-}$and Ifnar $2^{-1-}$ Aim $^{-1-}$ mice compared with WT animals (Figure 5-5C), which is consistent with a previous study [155], suggesting that circulating IL-17 and IL-12 may also contribute to host defense at this time point. Level of IFN $\gamma$ was reduced in Ifnar $2^{-/-}$Aim $2^{--}$but not Ifnar $2^{-/-}$mice. This could be due to a lack of IL-18-induced IFN $\gamma$ production in Ifnar2 ${ }^{-1-}$ Aim $^{-1-}$ mice (Figure 5-5C).

\section{Type I IFN Signaling Controls Activation of Apoptotic Caspases and Cell Death}

Type I IFN signaling has been shown to exert its detrimental effects to the host via induction of cell death during L. monocytogenes and Brucella abortus infection [50]. To determine whether type I IFN signaling promotes excessive cell death during $F$. novicida infection, we measured levels of apoptotic caspase- $8,-3$, and -7 in liver tissues of WT, Ifnar2 ${ }^{--}$, and Ifnar $2^{--}$Aim $2^{-/}$mice. In line with our hypothesis, the levels of cleaved caspase-8, -3 and -7 were lower in Ifnar $2^{--}$and Ifnar $2^{-1-}$ Aim $2^{-1-}$ mice than in WT mice 1 and 2 days after infection (Figure 5-6A-B). These results suggest that induction of apoptosis is impaired in the absence of type I IFN signaling. Levels of cleaved caspase-3 and -7 were similar between WT and $A i m 2^{---}$mice (Figure 5-7A). Additionally, Ifnar $2^{--}$and Ifnar $2^{-1-}$ Aim $2^{-1-}$ mice showed decreased cleavage of caspase-8, -3 and -7 on day 3 post infection (Figure 5-6C), whereas Aim $2^{-/}$mice had higher levels of cleaved caspase- $8,-3$ and -7 , possibly due to excessive liver damage caused by uncontrolled bacteria replication (Figure 5-6C). TUNEL staining also indicated reduced cell death in Ifnar2 ${ }^{-/-}$and Ifnar2 ${ }^{-/-}$Aim $^{-/-}$mice and enhanced cell death in Aim2 $2^{-/-}$mice after the infection (Figure 5-7B). We further tested role of AIM2 in mediating apoptosis in vitro during $F$. novicida infection by measuring cleavage of apoptotic caspases. Cleavage of caspase- 8 and -3 was increased in Aim $^{-/-}$BMDMs compared with WT cells after the infection (Figure 5-7C). A previous study has shown that the AIM2 inflammasome negatively regulates type I IFNs production [157]. It is possible that increased levels of type I IFNs in Aim2 ${ }^{-/}$BMDMs induces activation of caspase-8 and -3. Interestingly, caspase-7 activation was decreased in Aim $^{-/-}$BMDMs (Figure 5-7C). Caspsae-7 has been reported as a substrate of caspase-1 [158]. The reduced cleavage of caspase-7 in Aim $2^{-/}$BMDMs could be due to impaired caspase-1 activation during the infection. Overall, these results suggest that $\operatorname{Aim}^{-/-}$BMDMs have increased apoptosis after $F$. novicida infection. On the other hand, apoptosis occurred similarly in infected Aim $2^{-/}$mice initially but further increased at a later stage of the infection compared with 
A
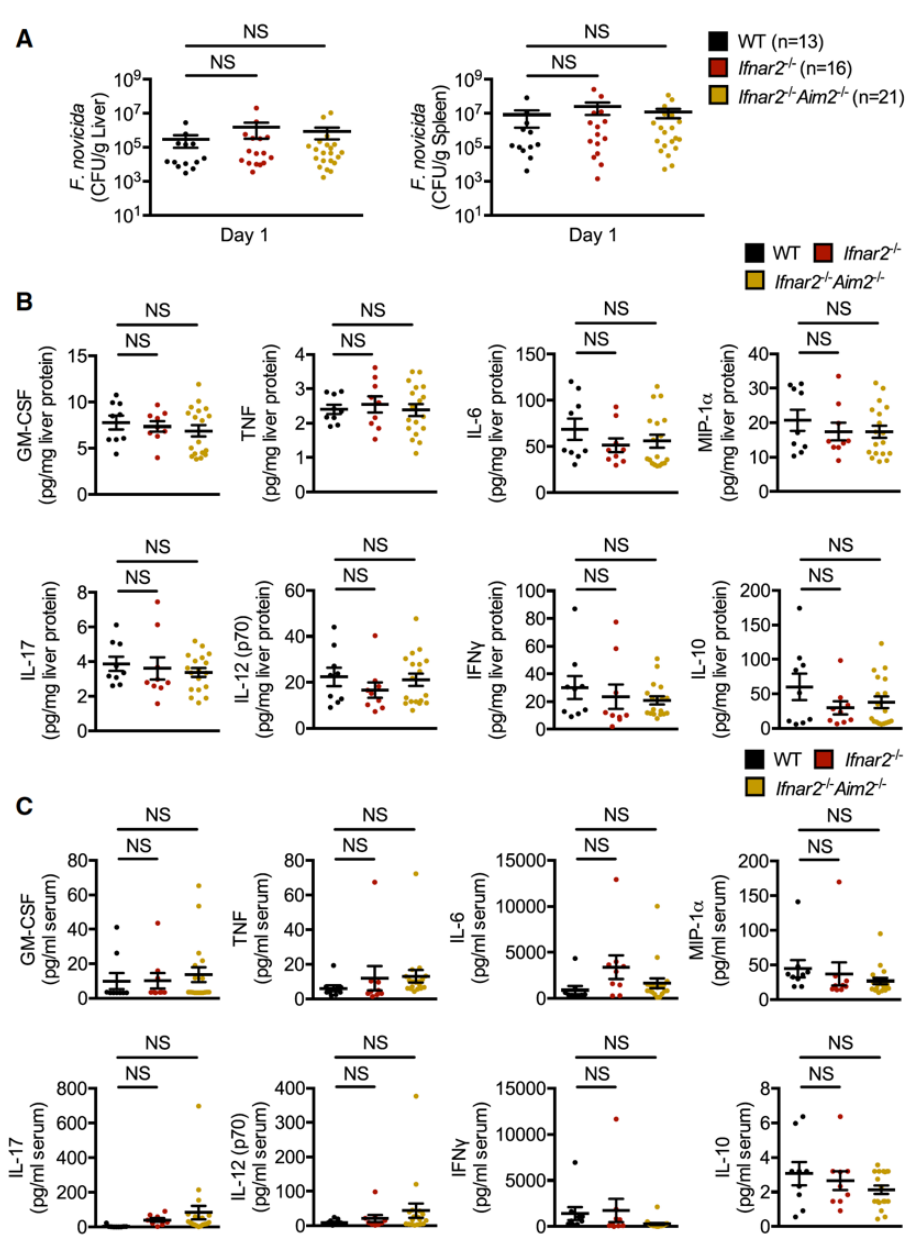

Figure 5-4. Type I IFN signaling does not alter production of a range of inflammatory cytokines during $F$. novicida infection.

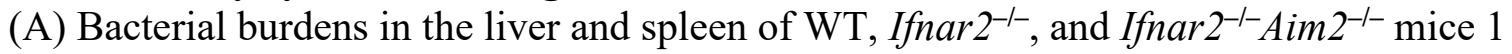
day after infection with $F$. novicida . (B and C) Concentrations of GM-CSF, TNF, IL-6, MIP1- $\alpha$, IL-17, IL-12 (p70), IFN $\gamma$, and IL-10 in the liver (B) or serum (C) of WT, Ifnar2${ }^{\prime-}$, and Ifnar $2^{-/-}$Aim $^{-/-}$mice 1 day after infection, as in (A). NS, not significant; one-way ANOVA with Dunnett's multiple-comparisons test. Data are pooled from two (B and C) or three $(\mathrm{A})$ independent experiments (mean and SEM in $\mathrm{A}-\mathrm{C}$ ). 
A

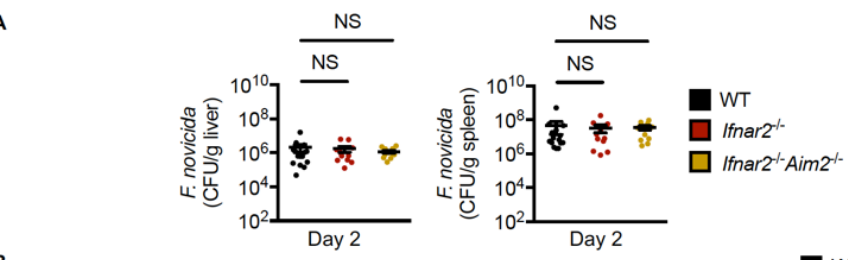

B

B WT $\square$ Ifnar2 $^{-1-}$
$\square$ Ifnar2 $^{-1}$ Aim2 $2^{-1-}$
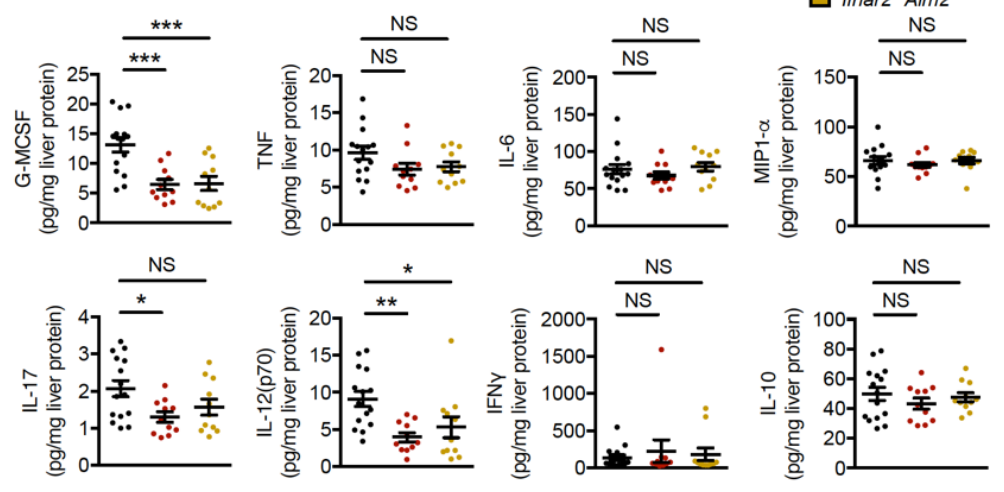

C
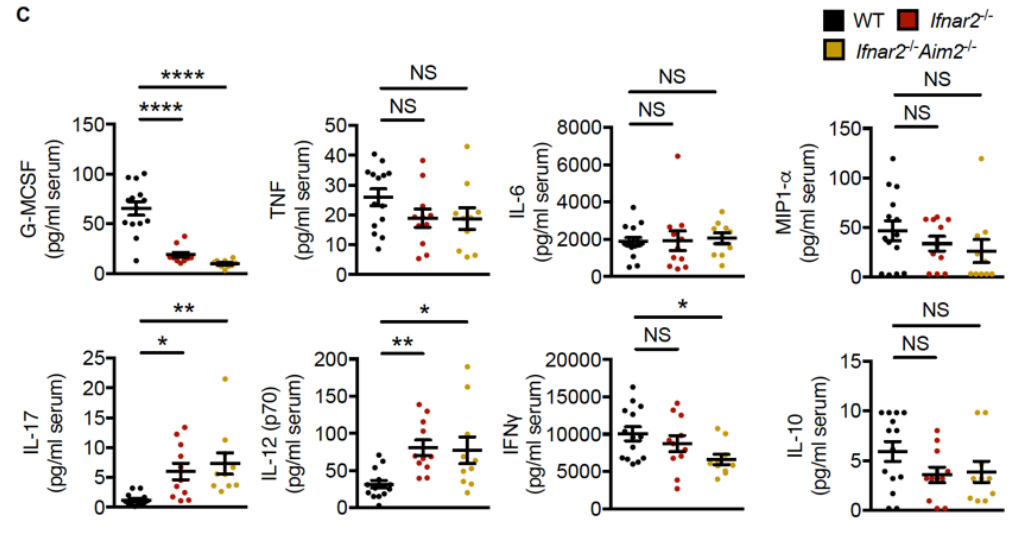

Figure 5-5. Inflammatory cytokine production after $F$. novicida infection.

(A) Bacterial burdens in the liver and spleen of WT, Ifnar2 $2^{-/-}$, and Ifnar $2^{-/-} \operatorname{Aim}^{-/-}$mice 2 days after infection with $F$. novicida. (B-C) Concentrations of GM-CSF, TNF, IL-6, MIP1- $\alpha$, IL-17, IL-12 (p70), IFN $\gamma$, and IL-10 in the liver (B) or serum (C) of WT, Ifnar $2^{-1}$, and Ifnar $2^{-1-}$ Aim $2^{-1-}$ mice 2 days after infection as in (A). NS, not significant; $* P<0.05 ; * * P<0.01, * * * P<0.001, * * * * P<0.0001$ (one-way ANOVA with Dunnett's multiple-comparisons test $[\mathrm{A}-\mathrm{C}]$ ). Data are pooled from two independent experiments (A-C; mean and SEM in A-C). 

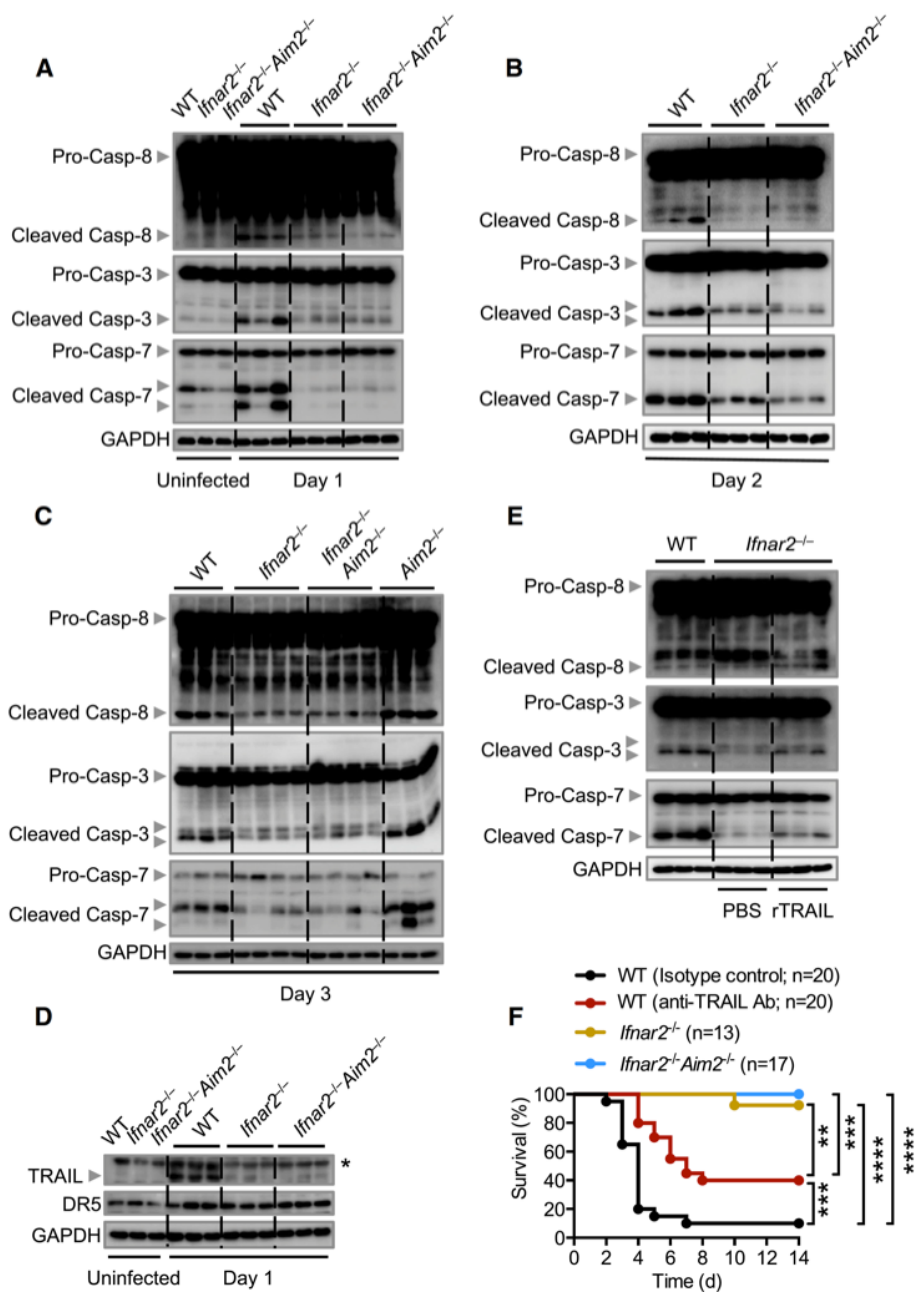

Figure 5-6. Type I IFN signaling induces apoptotic cell death.

(A-C) Immunoblot analysis of pro-caspase-8, cleaved caspase-8, pro-caspase-3, cleaved caspase-3, pro-caspase-7, cleaved caspase-7, and GAPDH in liver tissue from uninfected animals or WT, Ifnar $2^{-1-}$, and Ifnar $2^{-1-} A i m 2^{-/-}$mice 1 day (A) or 2 days (B) after $F$. novicida infection or from WT, Ifnar $2^{-/-}$, Ifnar $2^{-/-} A i m 2^{-l-}$, and $A i m 2^{-l-}$ mice 3 days (C) after $F$. novicida infection. (D) Immunoblot analysis of TRAIL, DR5, and GAPDH in liver tissues of uninfected animals or WT, Ifnar $2^{-1-}$, and Ifnar $2^{-1-}$ Aim $2^{-/-}$mice 1 day after infection. The asterisk indicates a non-specific band. (E) Immunoblot analysis of procaspase-8, cleaved caspase-8, pro-caspase-3, cleaved caspase-3, pro-caspase-7, cleaved caspase-7, and GAPDH in liver tissue from WT and Ifnar2/ mice treated with PBS or rTRAIL 1 day after $F$. novicida infection. (F) Survival of WT mice that received TRAIL neutralizing antibody or isotype control, Ifnar $2^{-/-}$mice, and Ifnar $2^{-1-} \mathrm{Aim}^{-/-}$mice infected with F. novicida. NS, not significant; **p $<0.01 ; * * * p<0.001$, ****p $<0.0001$; long-rank test $(\mathrm{F})$. Data are pooled from three independent experiments $(\mathrm{F})$ or are from one experiment representative of two (B and $\mathrm{E})$ or four $(\mathrm{A}, \mathrm{C}$ and $\mathrm{D})$ independent experiments. 

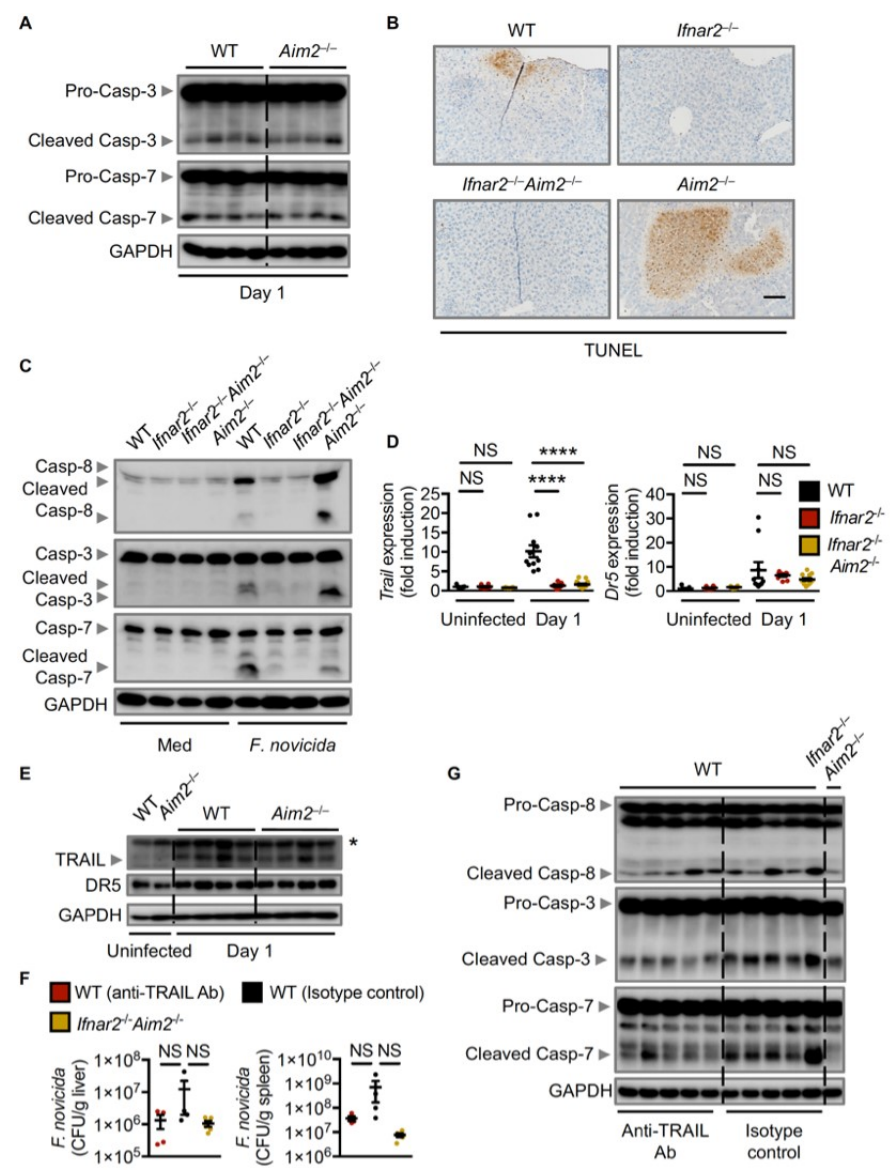

Figure 5-7. Analysis of apoptotic cell death and TRAIL pathway during $F$. novicida infection.

(A) Immunoblot analysis of cleavage of caspase-8, -3 and -7 and GAPDH in liver tissue from WT and Aim $2^{-/-}$mice 1 day after $F$. novicida infection. (B) TUNEL staining of liver tissues from WT, Ifnar2 $2^{--}$, Ifnar $2^{-/} \mathrm{Aim}^{-/-}$, and $\mathrm{Aim} 2^{-/-}$mice 3 days after $\mathrm{F}$. novicida infection. Scale bar, $100 \mu \mathrm{m}$. (C) Immunoblot analysis of cleavage of caspase-8, -3 and -7 and GAPDH in unprimed WT or mutant BMDMs left uninfected or assessed 20 hours after infection with $F$. novicida (MOI, 100). (D) Relative expression of genes encoding TRAIL and DR5 in liver tissues of uninfected animals or WT, Ifnar $2^{-/-}$, and Ifnar $2^{-1-}$ Aim $^{-/-}$mice 1 day after infection. (E) Immunoblot analysis of TRAIL, DR5 and GAPDH in liver tissue from uninfected animals or WT and $A \mathrm{im} 2^{-1-}$ mice 1 day after $F$. novicida infection. The asterisk indicates a non-specific band. (F) Bacterial burdens in the liver and spleen of WT mice that received TRAIL-neutralizing antibody or isotype control and Ifnar $2^{-/-}$Aim $2^{-/-}$mice 3 days after infection with F. novicida. (G) Immunoblot analysis of cleavage of caspase-8, -3 and -7 and GAPDH in liver tissue of WT mice that received TRAIL-neutralizing antibody or isotype control and Ifnar $2^{-1-}$ Aim $2^{-1-}$ mice after $F$. novicida infection. NS, not significant; $* * * * P<0.0001$ (one-way ANOVA with Dunnett's multiple-comparisons test [D and F]). Data are pooled from two experiments (D) or from one experiment representative of two (A, C and E) independent experiments or from one experiment (B, F and G; mean and SEM in D and F). 
WT mice. This could be explained by the cell-type specific differences in AIM2 function between BMDMs and liver where the majority of cells are hepatocytes. Similar to the liver tissues, BMDMs from Ifnar $2^{-/-}$and Ifnar $2^{-/}$Aim $^{-/-}$mice had reduced levels of cleaved caspase-8, -3 and -7 compared with WT cells following $F$. novicida infection (Figure 5-7C), indicating a potential role for type I IFN signaling in activating apoptotic caspases in vitro. The defective caspase-1 activation in Ifnar $2^{-/-}$and Ifnar $2^{-/} \mathrm{Aim}^{-/-}$ BMDMs may lead to impaired caspase-7 cleavage in these cells.

TRAIL is upregulated by type I IFNs and was shown to mediate type I IFN signaling-induced cell death during viral and bacterial infections [50]. TRAIL binds to its receptor DR5 and triggers apoptosis by driving caspase-8-mediated caspase-3 and -7 activation. We therefore hypothesize that type I IFN signaling may promote cell death through TRAIL pathway. We measured expression levels of TRAIL and DR5 in the liver and found that expression of TRAIL was upregulated in the liver of WT mice 1 day after infection; however, the induction was abrogated in Ifnar $2^{--}$and Ifnar $2^{-1-}$ Aim $^{-1-}$ mice

(Figure 5-6D, Figure 5-7D). DR5 was similarly induced between the animals (Figure 5-6D, Figure 5-7D). Interestingly, the level of TRAIL was slightly reduced in Aim $2^{-/}$mice after the infection, while the level of DR5 was similar (Figure 5-7E). IFN $\gamma$ can induce TRAIL expression in addition to type I IFNs (Park et al., 2004). Infected Aim $2^{-/-}$mice may exhibit reduced IFN $\gamma$ production (Pierini et al., 2013) which in turn leads to a modest reduction in TRAIL. However, the reduction was not significant enough to reduce apoptosis in $\mathrm{Aim} 2^{-/-}$mice, suggesting that other mechanisms such as Fas (CD95/APO-1) signaling may co-exist to promote marginal cell death. Furthermore, treatment of rTRAIL increased cleavage of caspase-8, -3 and -7 in Ifnar $2^{-1-}$ mice compared with PBS-treated Ifnar $2^{-/-}$mice after the infection (Figure 5-6E), indicating that type I IFN signaling induces TRAIL-mediated apoptosis during $F$. novicida infection. To further confirm that type I IFN signaling exacerbates $F$. novicida infection via the TRAIL pathway, we treated WT mice with TRAIL-neutralizing antibody or isotype control and infected and infected these mice along with Ifnar $2^{-/-}$and Ifnar $2^{-/}$ Aim $2^{-1-}$ mice with $F$. novicida. A higher dose of $F$. novicida was used in these experiments so that any protective effects rendered by TRAIL neutralization would be more prominent. Although $90 \%$ of WT mice that received isotype control died, only $60 \%$ of WT mice that received TRAIL-neutralizing antibody succumbed to infection over 14 days (Figure 5-6F). Neutralization of TRAIL also appeared to decrease bacterial burdens in the liver and spleen 3 days post infection (Figure 5-7F), demonstrating a detrimental role for TRAIL pathway during $F$. novicida infection in vivo. Moreover, WT mice that received TRAIL-neutralizing antibody had reduced levels of cleaved caspase-8, -3 and -7 compared with the control animals after the infection (Figure 5-7G), further confirming that TRAIL pathway leads to activation of apoptotic caspases during $F$. novicida infection.

In this study, we demonstrate type I IFN-induced apoptosis in the liver following $F$. novicida infection and the pathogenic effects of type I IFN signaling dominate the protective AIM2 responses (Figure 5-8). Type I IFN signaling has been shown to enhance susceptibility to L. monocytogenes and B. abortus infection by promoting cell death in the spleen with an ability to induce Trail expression [159-162]. Moreover, 


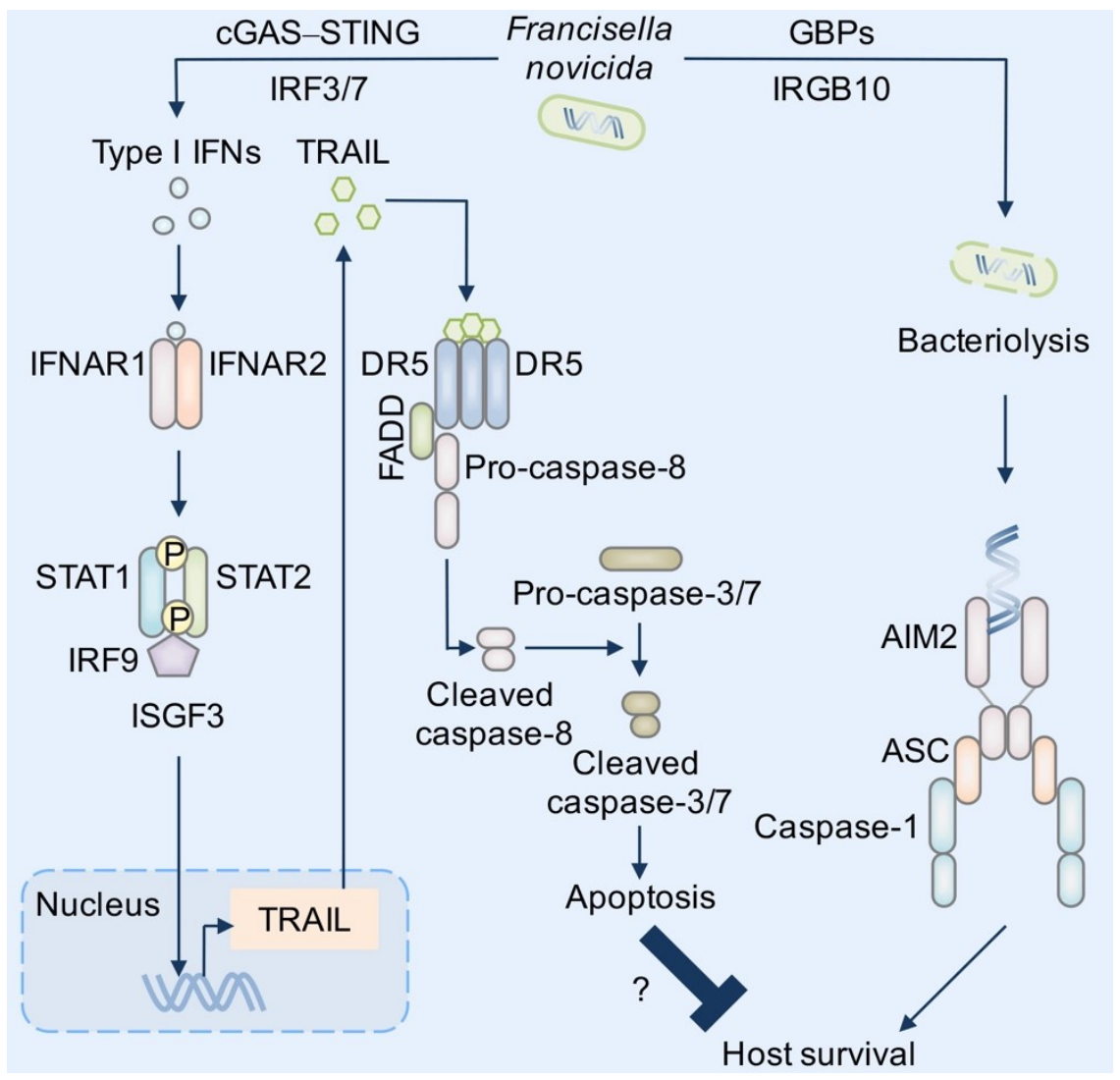

Figure 5-8. Opposing roles for type I IFNs signaling and AIM2 inflammasome responses during Francisella novicida infection in vivo.

cGAS-STING pathway induces production of type I IFNs in response to Francisella novicida infection in vivo, leading to induction of TRAIL and TRAIL-dependent extrinsic apoptotic cell death. On the other hand, AIM2 inflammasome is activated potentially via bacteriolysis of Francisella by GBPs and IRGB10. The detrimental type I IFN signaling overrides protective AIM2 inflammasome responses. 
Trail $^{--}$mice have decreased bacterial burdens in the liver and spleen and reduced splenic cell death after L. monocytogenes infection [163]. Increased apoptosis during F. novicida infection could lead to loss of hepatocytes, which have crucial roles in controlling infection [164]. An expansion of IL-17-producing $\gamma \delta \mathrm{T}$ cells is attributed to the phenotype of Ifnarl ${ }^{-/}$mice in a previous study [155]. Since reduced activation of apoptotic caspases in Ifnar $2^{-/-}$and Ifnar $2^{--}$A im $^{-/-}$mice occurred as early as day 1 post infection, preceding the elevation of IL-17 and IL-12 in the serum, it is intriguing to hypothesize that certain immune cells such as $\gamma \delta \mathrm{T}$ cells may exhibit dampened cell death in the absence of type I IFN signaling, leading to an increased production of circulating IL-17 and IL-12. Type I IFN signaling is necessary for activating AIM2 inflammasome in BMDMs infected with $F$. novicida via regulation of IRF1 and IRF1-mediated induction of GBPs and IRGB10 [150]. However, in contrast to mice lacking type I IFN signaling, mice lacking IRF1, GBPs or IRGB10 are susceptible to the infection due to impaired AIM2 inflammasome responses [44, 47]. Since activation of AIM2 inflammasome is IFNAR-independent in vivo, expression of IRF1, GBPs and IRGB10 might be intact in mice lacking type I IFN signaling because of the redundant induction by other types of IFNs. Consistent with this, priming Ifnar ${ }^{-/-}$BMDMs with IFN $\gamma$ bypasses the requirement for type I IFN signaling in the activation of the AIM2 inflammasome induced by F. novicida infection [45]. Overall, our work highlights the dominant role of type I IFN signaling, which exacerbates $F$. novicida infection in vivo, as opposed to the protective role of the AIM2 inflammasome. 


\section{CHAPTER 6. GASDERMIN D MEDIATES PROTECTION AGAINST FRANCISELLA NOVICIDA BY PROMOTING INFLAMMASOME ACTIVATION}

\section{Introduction}

Inflammasomes are innate immune multiprotein complexes formed by activation of innate immune sensors including NOD-like receptors, AIM2 or pyrin in response to pathogen-associated molecular patterns or danger-associated molecular patterns [63]. AIM2 is a cytoplasmic sensor for dsDNA. Upon activation, AIM2 assembles the inflammasome complex with adaptor ASC to mediate caspase-1 activation and caspase1 -dependent pyroptosis and release of pro-inflammatory cytokines IL-1 $\beta$ and IL-18 [32$35]$.

GSDMD was recently discovered as an executioner of pyroptotic cell death downstream of caspase-1 and caspase-11 [51-53]. GSDMD belongs to the gasdermin family of proteins and is maintained in an auto-inhibitory form under steady state. Inflammatory caspases including caspase-1 and -11 cleave at a link region between the $\mathrm{N}$ - and C-terminal of GSDMD, releasing the N-terminal fragment from inhibited by the C-terminal domain [51-53]. The N-terminal end of GSDMD binds to phosphoinositides located in the inner leaflet of the plasma membrane and forms pores on the membrane leading to cell lysis [54-58]. Recent studies have further shown that pores generated by GSDMD can function as channels to allow secretion of IL-1 $\beta$ and IL-18 independently of pyroptosis $[59,60]$.

AIM2 recognizes dsDNA originated from Gram-negative bacterium Francisella, DNA viruses MCMV and vaccinia virus and host cells, thereby exhibiting crucial roles in microbial infections and autoimmune diseases [41-43, 121, 165-167]. Mice lacking AIM2, ASC or caspase-1 are highly susceptible to infection by F. novicida, demonstrating a protective role for AIM2 inflammasome during the infection [41, 43-45, $47,83,168]$. IL-1 $\beta$ and IL-18 have been further shown to mediate the beneficial effects of AIM2 in response to Francisella tularensis Live Vaccine Strain [61, 62]. However, the role of pyroptosis downstream of AIM2 inflammasome is unknown. In this study, we showed that mice lacking the effector molecule of pyroptotic cell death, GSDMD, are susceptible to $F$. novicida infection. We further found that although cleavage of GSDMD is dependent on AIM2 inflammasome, GSDMD itself is required for optimal caspase-1 activation. Interestingly, GSDMD is also required for caspase-1 activation triggered by other AIM2 inflammasome stimuli including poly(dA:dT) transfection and MCMV infection. 


\section{Results}

\section{GSDMD Is Required for Host Defense Against $\boldsymbol{F}$. novicida Infection}

Mice lacking AIM2 inflammasome components such as AIM2 ( $\left.\mathrm{Aim}^{-/-}\right)$or

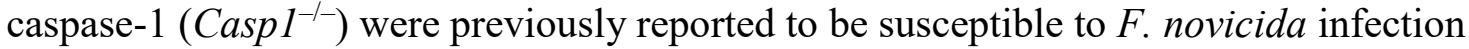
[41, 43-45, 47, 83, 168]. Consistent with previous findings, we found that $\mathrm{Aim}^{-/-}$and

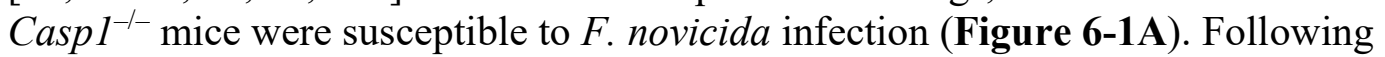
assembly of the inflammasome, activated caspase-1 cleaves pro-IL-1 $\beta$ and pro-IL-18 into mature forms and mediates their release from the cell. We therefore tested the role of IL$1 \beta$ and IL-18 during F. novicida infection. Similar to Aim $2^{-1-}$ and Casp $1^{-/-}$mice, mice lacking both IL-1R and IL-18 (Ill $\left.1 r^{--} I l 18^{--}\right)$were also highly susceptible and succumbed to death within 3 days of the infection (Figure 6-1A), highlighting a protective role for IL-1 $\beta$ and IL-18 downstream of inflammasome activation during $F$. novicida infection.

GSDMD mediates pyroptosis downstream of AIM2 inflammasome in response to DNA transfection [53]. To test the role of pyroptosis during $F$. novicida infection, we infected WT mice and mice lacking GSDMD (Gsdm ${ }^{-1-}$ ) with $F$. novicida and monitored the survival. While $70 \%$ of WT survived on day 14 post infection, only $10 \%$ of $\mathrm{Gs}_{\mathrm{s}} \mathrm{m} \mathrm{d}^{-/}$ mice survived during the course of infection (Fig. 1A). Furthermore, $G s d m d^{-1-}$ mice had significantly increased bacterial burden in the liver and spleen 3 days after $F$. novicida infection (Figure 6-1B), further confirming a protective role for GSDMD. Pores formed by GSDMD have been shown to mediate secretion of IL-1 $\beta$ and IL-18. Indeed, we observed reduced level of circulating IL-18 in $G s d m d^{-/}$mice compared to WT mice 1-day post infection (Figure 6-1C).

\section{GSDMD Is Necessary for Optimal Caspase-1 Activation by F. novicida}

Inflammasome activation is critical for host defense against $F$. novicida infection in vivo. To test whether inflammasome activation is affected in the absence of GSDMD, we generated bone marrow-derived macrophages (BMDMs) from WT, Gsdm $d^{-1-}$ and Aim $2^{--}$mice. The population of $\mathrm{CD} 11 \mathrm{~b}^{+} \mathrm{F} 4 / 80^{+}$macrophage was comparable between WT and $G s d m d^{-1-}$ BMDMs (Figure 6-2A), suggesting that there is no difference in the extent of macrophage differentiation. We then infected BMDMs from WT, Gsdmd $d^{-1-}$ and Aim $2^{-/-}$mice with F. novicida and measured cleavage of pro-caspase-1 into its active form (p20) following assembly of inflammasome. Interestingly, Gsdm $d^{-1-}$ BMDMs showed decreased activation of caspase- 1 compared to WT cells after $F$. novicida infection (Figure 6-3A), highlighting the requirement of GSDMD for inflammasome activation in response to $F$. novicida. Caspase-1 activation was reduced in $G s d m d^{-1-}$ BMDMs after infection with multiple MOIs of $F$. novicida (Figure 6-2B). In addition, we observed impaired IL-1 $\beta$ and IL-18 release in $F$. novicida-infected Gsdmd ${ }^{-1}$ BMDMs, potentially due to impaired caspase-1 activation along with defective pore formation (Figure 6-3B), whereas secretion of TNF was similar between the cells (Figure 6-4). GSDMD has been shown to mediate pyroptotic cell death upon AIM2 inflammasome 
A
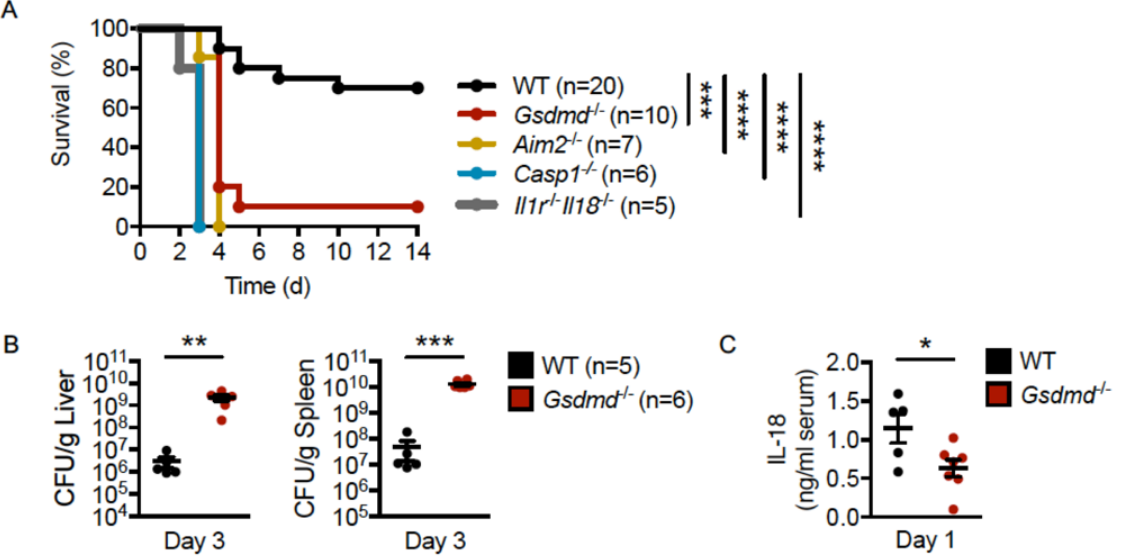

Figure 6-1. GSDMD contributes to host defense against $F$. novicida infection.

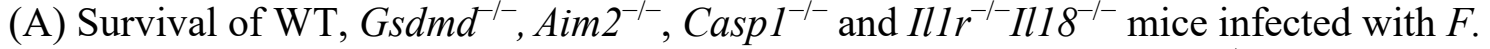
novicida. (B) Bacterial burden in the liver and spleen of WT and $G s d m d^{-1-}$ mice 3 days after infection with $F$. novicida. (C) Concentration of IL-18 in the sera of WT and $G s d m d^{-1-} 1$ day after the infection. $* P<0.05, * * P<0.01, * * * P<0.001, * * * * P<0.0001$ (logrank test $[\mathrm{A}]$ or two-tailed t test [B]). Data are pooled from two independent experiments (A) or from one experiment $(\mathrm{B}-\mathrm{C})$; mean and SEM in $\mathrm{B}-\mathrm{C})$. 

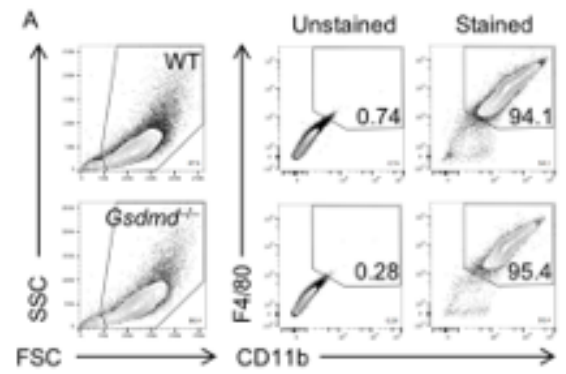

C
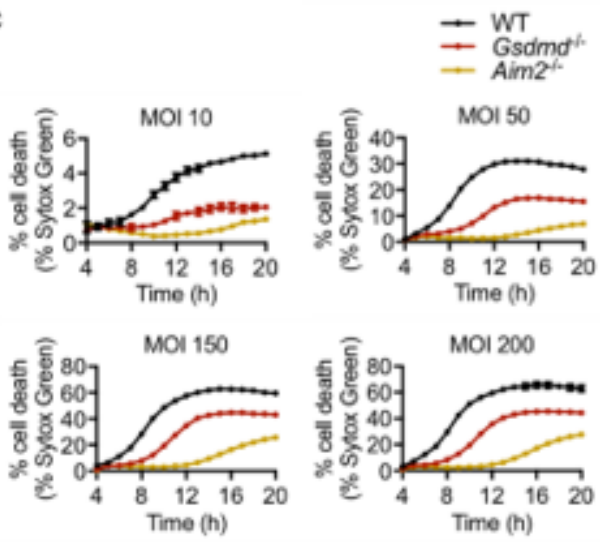
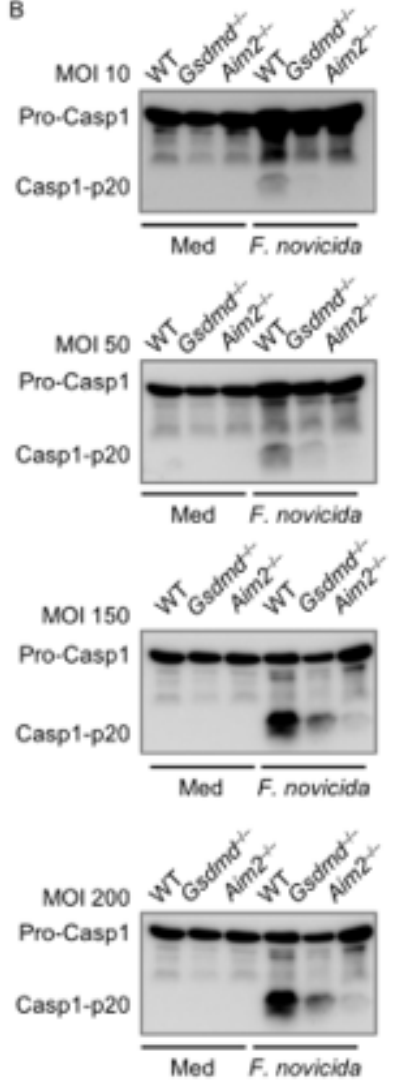

Figure 6-2. GSDMD regulates caspase-1 activation and cell death after various doses of $F$. novicida infection.

(A) Flow cytometric analysis of $\mathrm{CD} 11 \mathrm{~b}^{+} \mathrm{F} 4 / 80^{+} \mathrm{BMDMs}$ generated from $\mathrm{WT}$ and $G s d m d^{-1-}$ mice. (B) Immunoblot analysis of pro-caspase-1 (Pro-Casp-1) and the caspase1 subunit p20 (Casp-1 p20) in unprimed WT or mutant BMDMs left uninfected (medium alone [Med]) or assessed $20 \mathrm{~h}$ after infection with varying MOIs (10, 50, 150 or 200) of F. novicida. (C) Cell death analysis by Incucyte after infection as in (B). Data are representative of two independent experiments (A-C; mean and SEM in $\mathrm{C}$ ). 
A

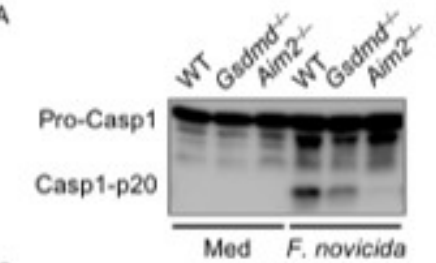

C

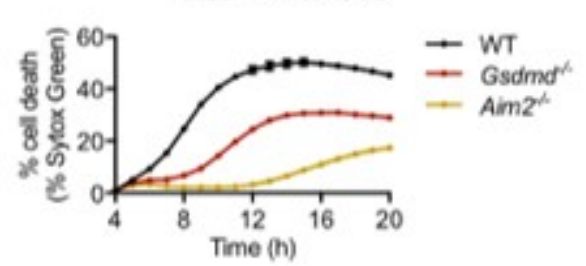

$\mathbf{E}$
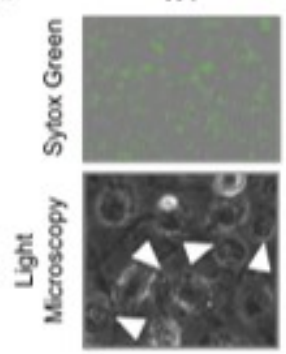
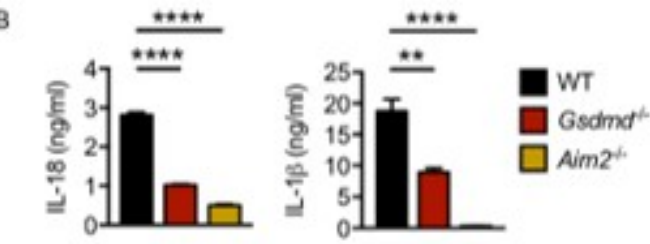

D
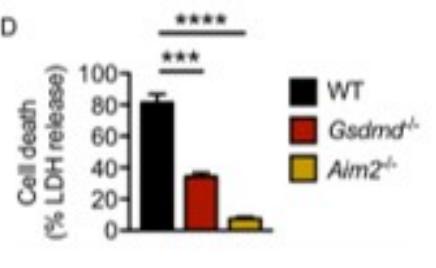

Figure 6-3. GSDMD promotes casaspse-1 activation by $\boldsymbol{F}$. novicida.

(A) Immunoblot analysis of pro-caspase-1 (Pro-Casp-1) and the caspase-1 subunit p20 (Casp-1 p20) in unprimed WT or mutant BMDMs left uninfected (medium alone [Med]) or assessed $20 \mathrm{~h}$ after infection with $F$. novicida (MOI, 100). (B) Release of IL-18 (left) and IL-1 $\beta$ (right) in unprimed BMDMs after infection as in (A). (C and D) Cell death analysis by Incucyte (C) or LDH release (D) after infection as in (A). (E) Representative images of Sytox Green staining by Incucyte (Scale bar, $100 \mu \mathrm{m}$ ) or light microscopy (Scale bar, $25 \mu \mathrm{m}$ ) of BMDMs after infection with $F$. novicida (MOI, 100). Arrowheads indicate representative pyroptotic cells. $* * P<0.01, * * * P<0.001, * * * * P<0.0001$ (one-way ANOVA with Dunnett's multiple comparisons test [B and D]). Data are representative of two (B), three (C, D and E) or six (A) independent experiments (mean and SEM in B, C and $\mathrm{D})$. 

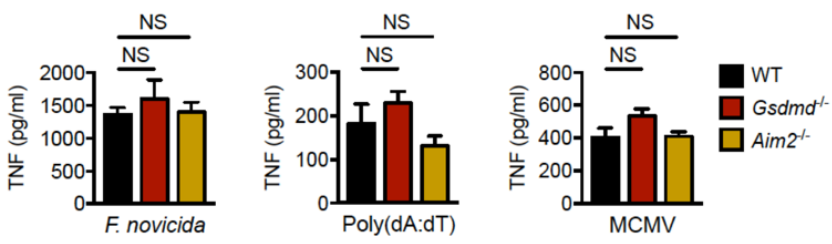

Figure 6-4. TNF release is normal in the absence of GSDMD.

Release of TNF in unprimed BMDMs assessed $20 \mathrm{~h}$ after infection with $F$. novicida (MOI, 100; left), $3 \mathrm{~h}$ transfection with poly(dA:dT) (middle), or $10 \mathrm{~h}$ infection with MCMV (right). NS, not significant (one-way ANOVA with Dunnett's multiple comparisons test). Data are representative of two independent experiments (mean and SEM). 
activation induced by cytoplasmic transfection of dsDNA [53]. To test the requirement of GSDMD in pyroptosis triggered by $F$. novicida infection, we first monitored the extent of cell death in WT, Gsdmd $d^{--}$and Aim ${ }^{-/-}$BMDMs after F. novicida infection using Sytox Green staining and found that $G s d m d^{-1-}$ BMDMs exhibited lower number of Sytox-Green positive cells than WT BMDMs over the course of the infection

(Figure 6-2C, Figure 6-3C, Figure 6-3E). Consistent with the Sytox green staining, LDH release was also decreased in $G s d m d^{-1-}$ BMDMs 20 hours post infection, which further confirms that pyroptosis in BMDMs during $F$. novicida infection is dependent on GSDMD (Figure 6-3D-E). Interestingly, GSDMD-independent cell death was also observed in Gsdmd ${ }^{-1-}$ BMDMs after F. novicida infection suggesting that other molecules may exist to mediate $F$. novicida-induced pyroptosis (Figure 6-2C, Figure 6-3C-E).

\section{GSDMD Is Necessary for Optimal Caspase-1 Activation by Other AIM2 Inflammasome Triggers}

In addition to $F$. novicida infection, AIM2 inflammasome can also be activated by transfection of poly(dA:dT) or MCMV infection. We found that caspase-1 activation induced by transfection of dsDNA ligand, poly(dA:dT) was partially reduced in $G s d m d^{-1-}$ BMDMs compared with WT cells (Figure 6-5A). Gsdmd ${ }^{-1-}$ BMDMs exhibited compromised release of IL-18 but not TNF (Figure 6-5B, Figure 6-4). Consistent with previous studies $[53,169]$, cell death was completely abolished in poly(dA:dT)transfected $G s d m d^{-1-}$ BMDMs during the early phase of stimulation (Figure 6-5C-E). Furthermore, MCMV-induced caspase-1 activation was dampened in Gsdmd ${ }^{-/-}$BMDMs compared with WT BMDMs (Figure 6-5F). Release of IL-1 $\beta$ and IL-18 in MCMVinfected $G s d m d^{-1-}$ BMDMs were impaired (Figure 6-5G), while TNF secretion was comparable between WT and Gsdmd ${ }^{-1-}$ BMDMs (Figure 6-4). Altogether, these data demonstrate that GSDMD is necessary for optimal caspase-1 activation in response to AIM2 inflammasome triggers including $F$. novicida and MCMV infection and, to a lesser extent, poly(dA:dT) transfection and that GSDMD plays a critical role in mediating pyroptosis after AIM2 inflammasome activation.

\section{AIM2 Inflammasome Cleaves GSDMD during $F$. novicida Infection}

Previous studies have shown that GSDMD is cleaved downstream of inflammasome activation. To test if AIM2 inflammasome is able to mediate GSDMD cleavage following $F$. novicida infection, we infected cells with $F$. novicida and observed impaired cleavage of GSDMD in BMDMs lacking AIM2 $\left(\mathrm{Aim}^{--}\right)$or ASC $\left(\mathrm{Asc}^{--}\right)$

(Figure 6-6A), suggesting that AIM2 inflammasome is required for GSDMD cleavage. Type I IFN signaling is required for activating AIM2 inflammasome in macrophages during $F$. novicida infection via induction of transcriptional factor IRF1, an interferonstimulated gene, which leads to IRF1-mediated expression of cell-autonomous immunity proteins including GBP2, GBP5 and IRGB10, leading to bacteriolysis and release of bacterial DNA [41-45, 47, 152, 155]. To investigate the involvement of GBPs in AIM2 mediated GSDMD cleavage, we utilized mice lacking multiple GBPs (including GBP2 
A

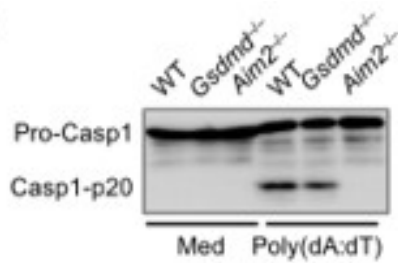

$\mathrm{C}$

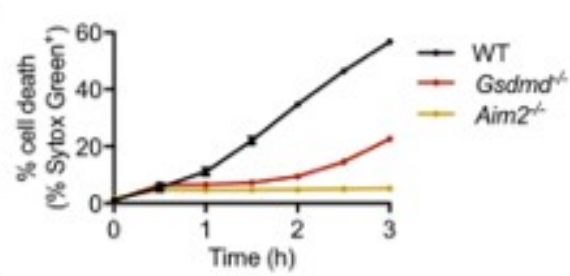

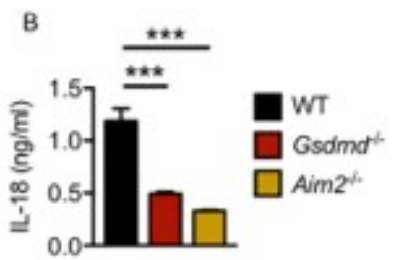

D

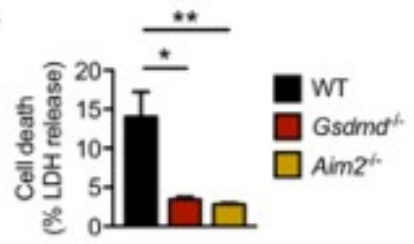

$E$

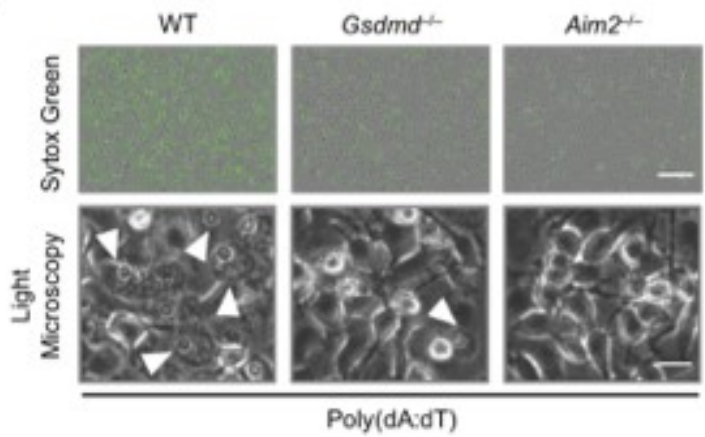

$\mathrm{F}$
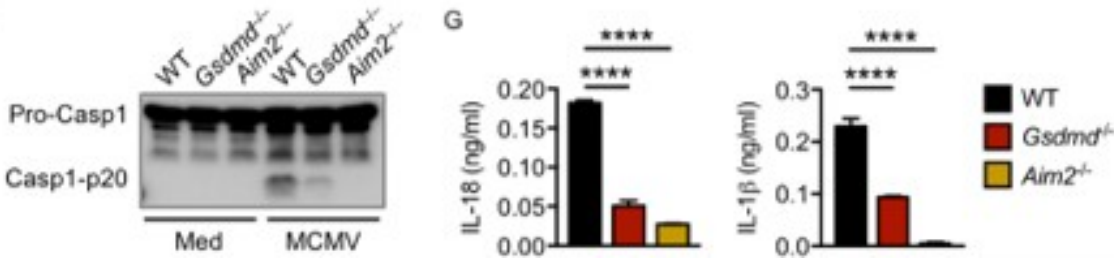

Figure 6-5. GSDMD is necessary for optimal casaspse-1 activation by other AIM2 inflammasome triggers.

(A) Immunoblot analysis of pro-caspase-1 (Pro-Casp-1) and the caspase-1 subunit p20 (Casp-1 p20) in unprimed WT or mutant BMDMs untreated (medium alone [Med]) or assessed $3 \mathrm{~h}$ after transfection with poly(dA:dT). (B) Release of IL-18 in unprimed BMDMs after treatment as in (A). (C and D) Cell death analysis by Incucyte (C) or LDH release (D) after treatment as in (A). (E) Representative images of Sytox Green staining by Incucyte (Scale bar, $100 \mu \mathrm{m}$ ) or light microscopy (Scale bar, $25 \mu \mathrm{m}$ ) of BMDMs after transfection with poly(dA:dT). Arrowheads indicate representative pyroptotic cells. (F) Immunoblot analysis of pro-caspase-1 (Pro-Casp-1) and the caspase-1 subunit p20 (Casp1 p20) in unprimed WT or mutant BMDMs left uninfected (medium alone [Med]) or assessed $10 \mathrm{~h}$ after infection with $10 \mathrm{MOI}$ of MCMV. (G) Release of IL-18 (left) and IL$1 \beta$ (right) in unprimed BMDMs after infection as in (F). ${ }^{*} P<0.01,{ }^{* *} P<0.01$, $* * * P<0.001, * * * * P<0.0001$ (one-way ANOVA with Dunnett's multiple comparisons test $[B, D$ and $G])$. Data are representative of two $(B, F$ and $G)$, three $(C, D, E)$ or six $(A)$ independent experiments (mean and SEM in B, C, D and G). 
A
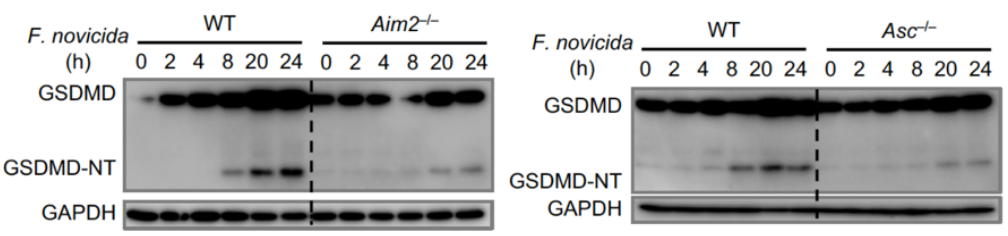

B

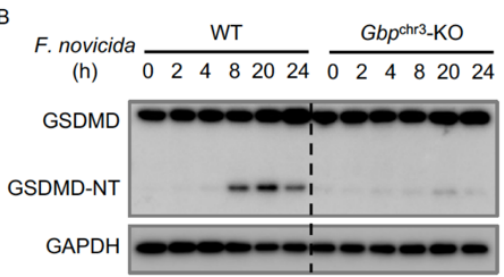

Figure 6-6. GSDMD cleavage is dependent on AIM2 inflammasome. (A and B) Immunoblot analysis of pro-GSDMD (GSDMD), cleaved N-terminal of GSDMD (GSDMD-NT) and GAPDH in unprimed WT, Aim $2^{--}$and $\mathrm{Asc}^{-/-}$BMDMs (A) or WT and $G b p^{\text {chr3 }}-$ KO BMDMs (B) infected with $F$. novicida (MOI, 20). Data are representative of two independent experiments (A and $\mathrm{B}$ ). 
and 5) encoded on chromosome $3\left(G b p^{\text {chr3 }}-\mathrm{KO}\right)$ and infected BMDMs from $G b p^{\text {chr3 }}-\mathrm{KO}$ mice with $F$. novicida. We found that $G b p^{\mathrm{chr}}-\mathrm{KO}$ BMDMs also exhibited reduced cleavage of GSDMD (Figure 6-6B). Overall, these data demonstrate that although caspase-1 activation induced by AIM2 inflammasome triggers is largely dependent on GSDMD, GSDMD cleavage still requires AIM2 inflammasome.

\section{Discussion}

Pyroptosis is a lytic form of cell death that is induced by inflammasomes leading to release of mature IL-1 $\beta$ and IL-18 and other cellular components including highmobility group box 1 (HMGB1) and IL-1 $\alpha$. It has not been discovered until recently that GSDMD induces pyroptosis by forming pores on plasma membrane after being activated by caspase- 1 or -11 [51-53]. In this study, we demonstrated a protective role for GSDMD during $F$. novicida infection. While caspase-1 processing is intact in the absence of GSDMD downstream of canonical NLRP3, NLRC4 or pyrin inflammasome [52, 53], we observed that activation of caspase-1 is impaired in $G s d m d^{-1-}$ BMDMs in response to AIM2 inflammasome stimuli. Given that a residual amount of caspase-1 activation was observed in $G s d m d^{-1}$ BMDMs infected with $F$. novicida, it is intriguing to hypothesize that during the early phase of $F$. novicida infection, caspase- 1 is activated in a GSDMD independent manner. Activated caspase- 1 may cleave GSDMD to allow the N-terminal to target $F$. novicida in the cytosol leading to bacteriolysis and release of bacterial DNA, which potentially amplifies activation of AIM2 inflammasome and further GSDMD cleavage at later phase of $F$. novicida infection in a positive feedback manner. Indeed, GSDMD has been shown to be associated with phosphoinositides and cardiolipin $[54,56$, 57], the latter of which is a component of $F$. novicida membrane as well as mitochondria [170]. Moreover, incubation of the N-terminal fragment of GSDMD with E. coli or Staphylococcus aureus in vitro leads to reduced bacterial CFU [57]. Furthermore, higher CFU of Listeria monocytogenes is observed in GSDMD-knocked down BMDMs [57], revealing a bactericidal role for GSDMD during intracellular bacterial infection $[56,57]$.

Consistent with this hypothesis, we also observed a higher bacterial burden in $G s d m d^{-1-}$ mice after $F$. novicida infection. Polymers that facilitate transfection of poly(dA:dT) or viral particles may be targeted by GSDMD similarly as F. novicida, which explains a reduction in caspase- 1 activation in the absence of GSDMD in response to poly $(\mathrm{dA}: \mathrm{dT})$ transfection and MCMV infection. In addition to the possibility that GSDMD targets bacterial membrane, GSDMD-mediated pore formation on plasma membrane at early phase of $F$. novicida infection may act in a feed forward loop giving an un-identified secondary signal for AIM2 inflammasome activation. It is also possible that GSDMD binds to cardiolipin which is located at inner-membrane of mitochondria. Pores may form by GSDMD on mitochondria inducing mitochondria damage and release of mitochondria DNA or other mitochondria-associated molecules into cytosol, serving as a potential source for AIM2 inflammasome activation. In addition, although levels of cell death and release of IL-1 $\beta$ and IL-18 are greatly reduced in $G s d m d^{-1-}$ BMDMs stimulated with AIM2 inflammasome triggers compared with WT cells, the levels are still higher than those in Aim $2^{-/-}$BMDMs, suggesting that additional mechanisms may 
mediate pyroptosis and pyroptosis-dependent IL-1 $\beta$ and IL-18 release downstream of AIM2 inflammasome activation. Consistent with our findings, GSDMD-independent cell death and cytokine release are also reported previously [52, 169]. It is intriguing to hypothesize that other members of GSDMD family such as GSDME can be activated by AIM2 to induce pore formation on cell membrane leading to pyroptosis [171, 172]. Altogether, our study highlights a critical role for GSDMD in mediating host defense against $F$. novicida infection and further underscores a requirement for GSDMD in promoting optimal caspase-1 activation following AIM2 inflammasome triggers. 


\section{CHAPTER 7. DISTINCT REGULATORY MECHANISMS CONTROL PROINFLAMMATORY CYTOKINES IL-18 AND IL-1 $\beta^{*}$}

\section{Introduction}

IL-18 and IL-1 $\beta$, members of the IL-1 cytokine family, are important mediators of inflammatory diseases and play critical roles in infection and cancer $[65-67,173]$. Unlike other cytokines, cellular IL-18 and IL-1 $\beta$ are synthesized as precursor proteins and need to be cleaved to generate their biologically active forms. Inflammasome, a multimeric protein complex, is a central regulator of this process by which bioactive IL-18 and IL-1 $\beta$ are generated. The inflammasome comprises an innate immune sensor that includes the NOD-like receptor, AIM2-like receptor, and pyrin; the adaptor protein ASC; and the cysteine protease caspase 1 [36]. Inflammasome assembly induces the activation of caspase-1, which mediates the proteolytic processing of pro-IL-18 and pro-IL-1 $\beta$ to generate their bioactive forms and their release from the cell [36].

IL-18 is constitutively expressed in blood monocytes and intestinal epithelial cells of healthy humans $[68,174]$. It is also expressed in murine macrophages, dendritic cells, endothelial cells, intestinal epithelial cells, and keratinocytes under steady state [175177]. However, IL-1 $\beta$ is not constitutively expressed under homeostasis. IL-1 $\beta$ expression is induced in blood mononuclear cells, macrophages, and dendritic cells during stimulation with TLR ligands and other cytokines (e.g., TNF) [65-67]. Unlike IL$1 \beta$, a cellular pool for IL-18 already exists before an inflammatory stimulus, and is ready to be activated and released by the inflammasome [64]. It must however be noted that LPS-mediated priming of NLRP3 is required for inflammasome activation and subsequent release of both IL-18 and IL-1 $\beta$ [178]. However, several studies suggest that despite being constitutively expressed, IL-18 can also be induced under certain circumstances. Treatment with LPS (TLR4 agonist) has been shown to upregulate the mRNA levels of Il18 [68, 69]. Incubation of CpG oligonucleotides (TLR9 agonist) with dendritic cells or infection of monocytes with the Sendai virus can also increase the expression of 1118 [70, 71].

The mechanisms by which IL-18 and IL-1 $\beta$ levels are regulated by different inflammatory signals remain unclear. In this study, we showed that expression of IL-18 is induced and that the increased level is sustained during TLR4, TLR2, or TLR7 ligands stimulation. In contrast, IL-1 $\beta$ expression declines soon after reaching its peak level. The TLR3 and cGAS-STING pathways induced IL-18 but were modest at inducing IL-1 $\beta$ expression. Importantly, type I IFN signaling was required to upregulate IL-18 in response to all stimuli tested. Thus, in macrophages lacking components of type I IFN signaling, IL-18 was not induced by any inflammatory stimuli. Together, our findings

\footnotetext{
* Adapted with permission. Zhu, Q. and T.D. Kanneganti, Cutting Edge: Distinct Regulatory Mechanisms Control Proinflammatory Cytokines IL-18 and IL-1 $\beta$. J Immunol, 2017. 198(11): p. 4210-4215. Copyright 2017. The American Association of Immunologists, Inc.
} 
demonstrate that IL-18 and IL-1 $\beta$ expression are differentially regulated. Expression of these inactive pro-inflammatory cytokines is an essential step for their maturation into bioactive forms by caspase-1 inflammasomes. Overall, our data demonstrating differential regulation of IL-18 and IL- $1 \beta$ will be fundamental to understanding several inflammatory disease settings.

\section{Results and Discussion}

\section{IL-18 and IL-1ß Are Differentially Induced in Response to Inflammatory Stimuli}

TLRs, one of the major types of pattern recognition receptors in the cell [179], induce expression of pro-inflammatory cytokines and type I IFNs in response to pathogen-associated molecular patterns and damage-associated molecular patterns [179]. We first confirmed that IL-18 was constitutively expressed in untreated WT BMDMs, similar to previous reports $[68,175]$. Interestingly, we observed that its expression was upregulated by the TLR4 ligand LPS (Figure 7-1A). As expected, $I l l b$ was not expressed in untreated BMDMs but was induced shortly after LPS treatment (Figure 7-1A). Interestingly, although gene and protein expression of IL-18 was sustained 24 hours after stimulation, that of IL- $1 \beta$ was induced initially but reduced substantially at 24 hours (Figure 7-1A). These results suggest that the expressions of IL-18 and IL-1 $\beta$ are differentially regulated after LPS stimulation.

After binding to TLR4, LPS signals through both the MyD88 and TRIF pathways [179]. To determine the specific roles of MyD88 and TRIF pathways in regulating IL-18 and IL-1 $\beta$ expression, we treated cells with ligands that signal through either the MyD88 or TRIF pathway. Interestingly, Pam3CSK4, which signals through the MyD88 pathway downstream of TLR2 induced sustained expression of IL-18 over the treatment period in WT BMDMs (Figure 7-1B). On the other hand, IL-1 $\beta$ was upregulated 4 hours after treatment but diminished dramatically at later time points (Figure 7-1B). Gardiquimod induces the MyD88 pathway downstream of TLR7. Induction kinetics of mRNA expression and protein levels of IL-18 and IL-1 $\beta$ in WT BMDMs treated with gardiquimod were similar to those treated with Pam3CSK4 (Figure 7-2). Poly(I:C) is sensed by TLR3 to activate the TRIF pathway. Poly(I:C) treatment induced Ill8 expression in WT BMDMs. It also induced $I l 1 b$ expression, although at a much lower fold induction than by LPS, Pam3CSK4, and gardiquimod (Figure 7-1C). These findings suggest that the TRIF pathway moderately induces $I l l b$ expression, and both the MyD88 and TRIF pathways induce $I l 18$ expression. Taken together, our results show that IL-18 and IL-1 $\beta$ can be induced by various TLR ligands. Further, during chronic TLR stimulation, IL-18 expression is sustained but IL-1 $\beta$ expression is downregulated. These data suggest distinct regulatory pathways that control the expression of pro-IL-18 and pro-IL-1 $\beta$ levels during chronic TLR stimulation.

One of the prominent mediators induced by the TRIF pathway are type I IFNs, including IFN $\beta$ [179]. Poly(I:C) induced the expression of $I l 18$ comparable to LPS 

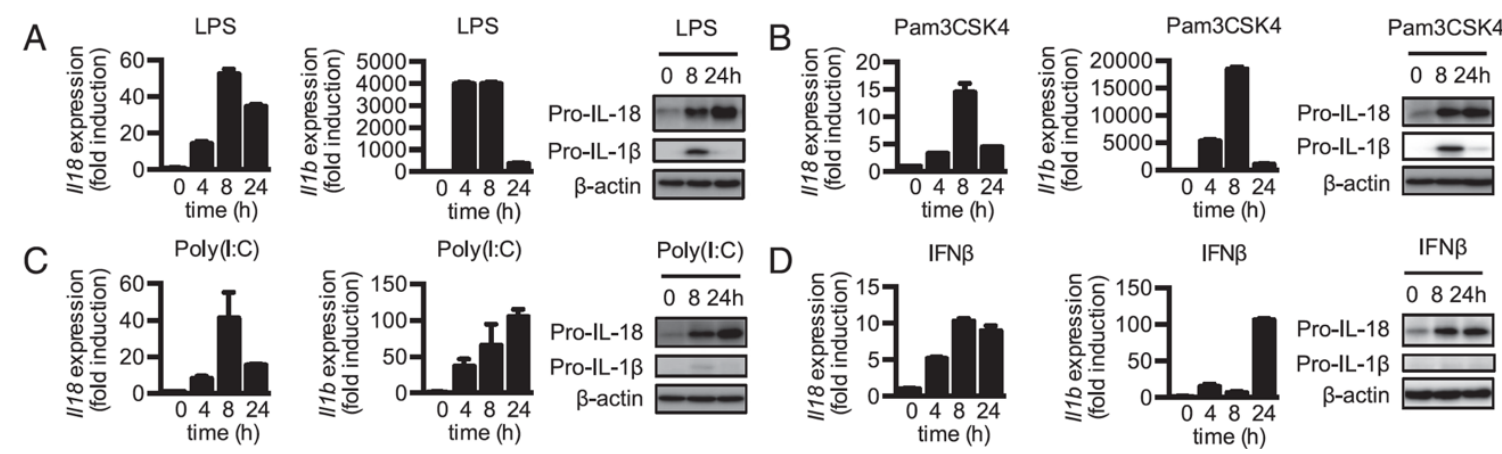

Figure 7-1. IL-18 and IL-1 $\beta$ are differentially expressed in response to inflammatory stimuli.

(A-D) Real-time qPCR analysis of genes encoding IL-18 and IL-1 $\beta$ and immunoblot analysis of pro-IL-18, pro-IL-1 $\beta$, and $\beta$-actin (loading control) in WT BMDMs at various time points after LPS (A), Pam3CSK4 (B), poly(I:C) (C), or IFN- $\beta$ (D) treatment. Data are representative of three independent experiments

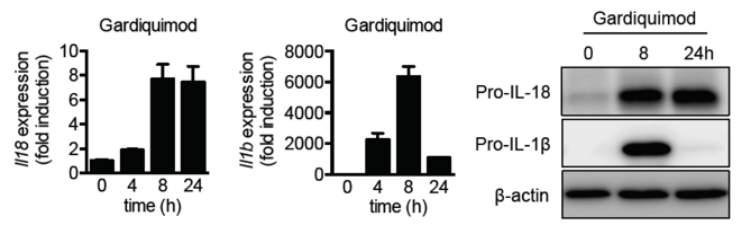

Figure 7-2. Kinetics of IL-18 and IL-1 $\beta$ expression stimulated by gardiquimod. (A) Real-time qPCR analysis of the genes encoding IL-18 and IL-1 $\beta$ and immunoblot analysis of pro-IL-18, pro-IL-1 $\beta$ and $\beta$-actin (loading control) in WT BMDMs at various times after treated with gardiquimod. Data are representative of three independent experiments. 
stimulation, which led us to hypothesize that the induction of $I 118$ requires type I IFNs. To determine the role of type I IFNs in modulating the expressions of $I l 18$ and $I l l \mathrm{~b}$, we treated WT BMDMs with recombinant IFN $\beta$ and measured $I l 18$ and $I l l b$ expression. IL18 mRNA and protein levels were induced after IFN $\beta$ treatment, but IL-1 $\beta$ mRNA and protein levels were only modestly induced at levels similar to those seen for Poly(I:C) stimulation (Figure 7-1C-D). Overall, these data suggest that treatment of type I IFNs upregulates IL-18 expression.

\section{Induction of IL-18 but Not IL-1ß Is Dependent on Type I IFN Signaling}

To test whether type I IFN signaling was required for induction of $I l 18$ expression by inflammatory stimuli, we treated WT BMDMs and cells lacking the subunit of type I IFN receptor IFNAR1 (Ifnar ${ }^{-1-}$ ) with LPS and monitored the expression of $I l 18$ and $I l l b$ over the treatment period. LPS treatment led to upregulation of IL-18 in WT BMDMs as early as 4 hours after treatment. IL-18 expression further increased and was sustained during chronic LPS treatment (Figure 7-3A). Surprisingly, this induction of IL-18 by LPS was impaired in Ifnarl $^{-/-}$cells (Figure 7-3A), indicating that type I IFN signaling is crucial to induce IL-18 during LPS treatment. In WT BMDMs, IL-1 $\beta$ expression increased 4 hours and 8 hours after treatment but diminished at later time points (Figure 7-3A). Unlike $I l 18$, induction of IL- $1 \beta$ remained largely intact in $I f n a r I^{-/-}$cells (Figure 7-3A). Moreover, expression of $I l l b \mathrm{mRNA}$ and protein was higher in $I f n a r I^{-/}$ BMDMs than in WT BMDMs 16 hours and 24 hours after stimulation (Figure 7-3A), suggesting that type I IFN signaling may negatively regulate $I l l b$ expression during chronic LPS stimulation.

To further test whether the induction of IL-18 by stimuli that trigger only the MyD88 or TRIF pathway also depends on type I IFN signaling, we treated WT and Ifnar $^{-/-}$BMDMs with Pam3CSK4, gardiquimod, or Poly(I:C). Induction of IL-18 was abolished in Ifnar $1^{-/-}$BMDMs compared with WT BMDM when MyD88 signaling was triggered by Pam3CSK 4 or gardiquimod treatment (Figure 7-3B-C). However, IL-1 $\beta$ expression remained unchanged in Ifnar $1^{-/-}$BMDMs after either stimulation (Fig. 2B and 2C). Further, during treatment with Poly(I:C), which signals via the TRIF pathway, IL-18 induction was impaired in Ifnar ${ }^{-/}$BMDMs compared with WT BMDMs (Figure 7-3D). Recognition of cytosolic DNA by the cGAS-STING pathway results in the production of type I IFNs [10]. We hypothesized that stimuli that induce the production of type I IFNs will promote IL-18 expression that is dependent on type I IFN signaling. Therefore, we activated the cGAS-STING pathway by transfecting double-stranded DNA, poly(dA:dT), or the STING ligand cGAMP in BMDMs. Expression of $I l 18$ was increased by activation of the cGAS-STING pathway (Figure 7-3E-F), but that of $I l l b$ was not robustly induced (Figure 7-3E-F). As expected, the induction of $I l 18$ was lower in Ifnar $1^{-1-}$ BMDMs in response to poly(dA:dT) or cGAMP than in WT BMDMs (Figure 7-3E-F). Collectively, these results suggest that type I IFN signaling is critical for induction of IL-18, but not IL-1 $\beta$ after inflammatory stimulations. 

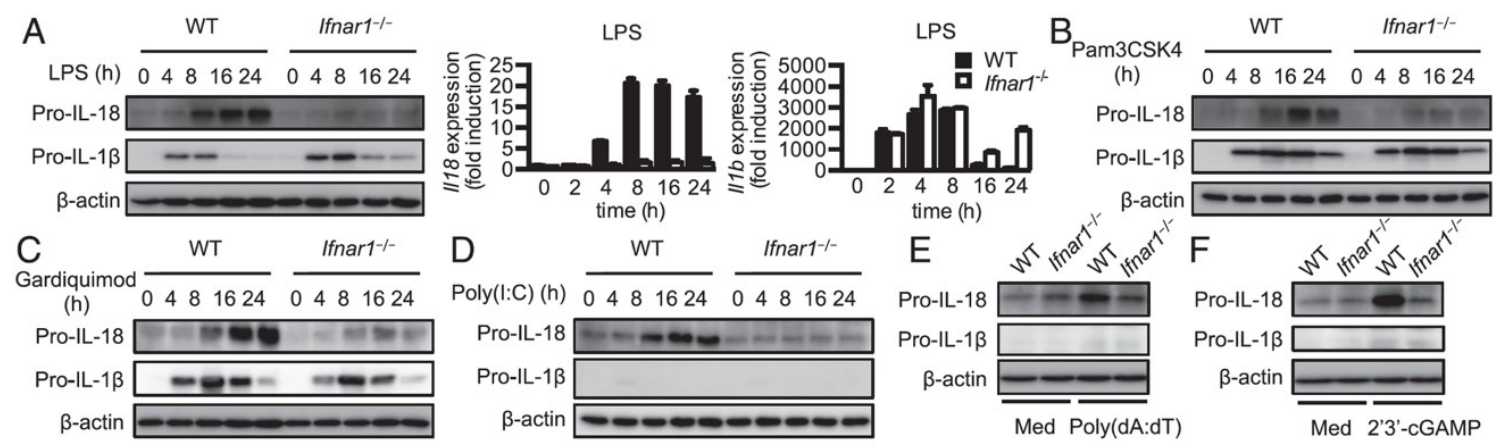

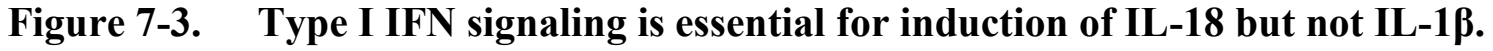
(A) Immunoblot analysis of pro-IL-18, pro-IL-1 $\beta$, and $\beta$-actin (loading control) and realtime qPCR analysis of genes encoding IL-18 and IL-1 $\beta$ in WT or Ifnar $1^{-1-}$ BMDMs at various time points after LPS treatment. (B-D) Immunoblot analysis of pro-IL-18, proIL-1 $\beta$, and $\beta$-actin (loading control) in WT or Ifnar ${ }^{-1-}$ BMDMs at various time points after Pam3CSK4 (B), gardiquimod (C), or poly(I:C) (D) treatment. (E and F) Immunoblot analysis of pro-IL-18, pro-IL-1 $\beta$, and $\beta$-actin (loading control) in untreated WT or Ifnar $1^{-1-}$ BMDMs (medium alone [Med]) or BMDMs at $8 \mathrm{~h}$ after transfected with poly(dA:dT) (E) or 2'3'-cGAMP (cGAMP) (F). Data are representative of three independent experiments. 


\section{Components of Type I IFN Signaling Are Essential to Induce IL-18}

The type I IFN receptor is a heterodimeric complex formed by IFNAR1 and IFNAR2 [180]. Binding of type I IFN to IFNAR1 and IFNAR2 induces the formation of ISGF3 by STAT members STAT1, STAT2, and IRF9, which function as transcriptional factors to induce the expression various interferon-stimulated genes (ISGs) [180]. To test whether other components of type I IFN signaling are also required to induce IL-18, we first measured IL-18 and IL-1 $\beta$ levels in WT BMDMs and cells lacking IFNAR2, IRF9, or STAT1 (Ifnar $2^{-/-}, \operatorname{Irf9^{-/-}}$ and Stat1 ${ }^{-/}$, respectively) after LPS treatment. Similar to Ifnar $1^{-/-}$BMDMs, induction of IL-18 was also impaired in Ifnar2 $2^{-/-}$, Irf $^{-/-}$, and Stat $1^{-/-}$ BMDMs (Figure 7-4A). However, expression of $1 l 1 \mathrm{~b}$ mRNA and protein was not compromised but showed a slight increase at 16 hours and 24 hours after stimulation in Ifnar $2^{-/}$, Irf $9^{-/-}$, and Stat $^{-/-}$BMDMs (Figure 7-4A). Moreover, pro-IL-18 was not upregulated in Ifnar2 ${ }^{-/-}$, Irf9 ${ }^{-/-}$, and Stat $1^{-1-}$ BMDMs treated with Pam3CSK4 or Poly(I:C) compared with WT BMDMs (Figure 7-4B-C). ISGF3 directly binds to promoters of ISGs to induce their expression, some of which include IRFs that function as transcriptional factors to drive the expression of additional genes [181]. To test whether IL-18 was induced via ISGF3 or other IRFs downstream of the type I IFN receptor, we stimulated Ifnar $1^{-/-}$, Ifnar $2^{-/-}$, Irf $9^{-/-}$, and Stat $^{-/-}$BMDMs with recombinant IFN $\beta$ and observed that IFN $\beta$-induced expression of $I l 18$ was compromised in Ifnar $1^{-/}$, Ifnar2 $2^{-/-}$, Irf9 ${ }^{-/-}$, and Stat1 $1^{-/}$BMDMs compared with WT cells

(Figure 7-5A). On the other hand, the induction of Il18 by IFN $\beta$ was not affected in BMDMs lacking other IRFs, including IRF1, IRF3, IRF4, IRF5, IRF7, or IRF8 (Irf1 ${ }^{-/}$, $\operatorname{Irf3}{ }^{-/-}, \operatorname{Irf4}{ }^{\mathrm{fl} / \mathrm{fl}}-\mathrm{Lysm}-\mathrm{Cre}, \operatorname{Irf5} 5^{-/-}, \operatorname{Irf7^{--}}$ and $\operatorname{Irf8^{-/-}}$, respectively; Figure 7-5B-C), suggesting that induction of 1118 is dependent on ISGF3 but not other IRFs.

We have established that although both IL-18 and IL-1 $\beta$ belong to the IL-1 family and are activated by the inflammasome, IL-18 and IL-1 $\beta$ are differentially regulated (Figure 7-5D). Indeed, previous report has shown that secretion of IL-1 $\beta$ but not IL-18 from murine dendritic cells in response to Listeria monocytogenes p60 protein requires ROS production and caspase 11 [182]. Distinctive expression patterns of IL-18 and IL-1 $\beta$ are observed in the intestine. IL-18 is expressed at high level in colon and confers protection against inflammation by promoting epithelial cell proliferation and tissue repair [183]. IL-1 $\beta$, on the other hand is expressed at lower levels basally, but enhanced during acute inflammation [183]. Given that type I IFNs are protective against DSSinduced colitis [184], it is possible that the type I IFNs contribute to protection by upregulating the expression of $I l 18$ in the colon. Activation of the cGAS-STING pathway leads to the production of type I IFN [10]. Colon tissues from mice lacking STING also have decreased Il18 mRNA and protein expression after DSS treatment $[143,185]$. Therefore, type I IFN signaling can be explored as a therapeutic target in IL-18associated diseases. We further demonstrated that $I l 1 b$ gene and protein expression was reduced during chronic TLR stimulation in WT BMDMs, indicating that the transcription of $I l 1 b$ or stability of $I l 1 b$ mRNA may be compromised. Reduced translational activity or protein stability can also contribute to decreased levels of pro-IL-1 $\beta$. Indeed, there is translational inhibition of $I l 1 b$ expression in LPS-stimulated macrophages [186]. Moreover, pro-IL-1 $\beta$ is ubiquinated by E2 conjugase UBE2L3 and subsequently 


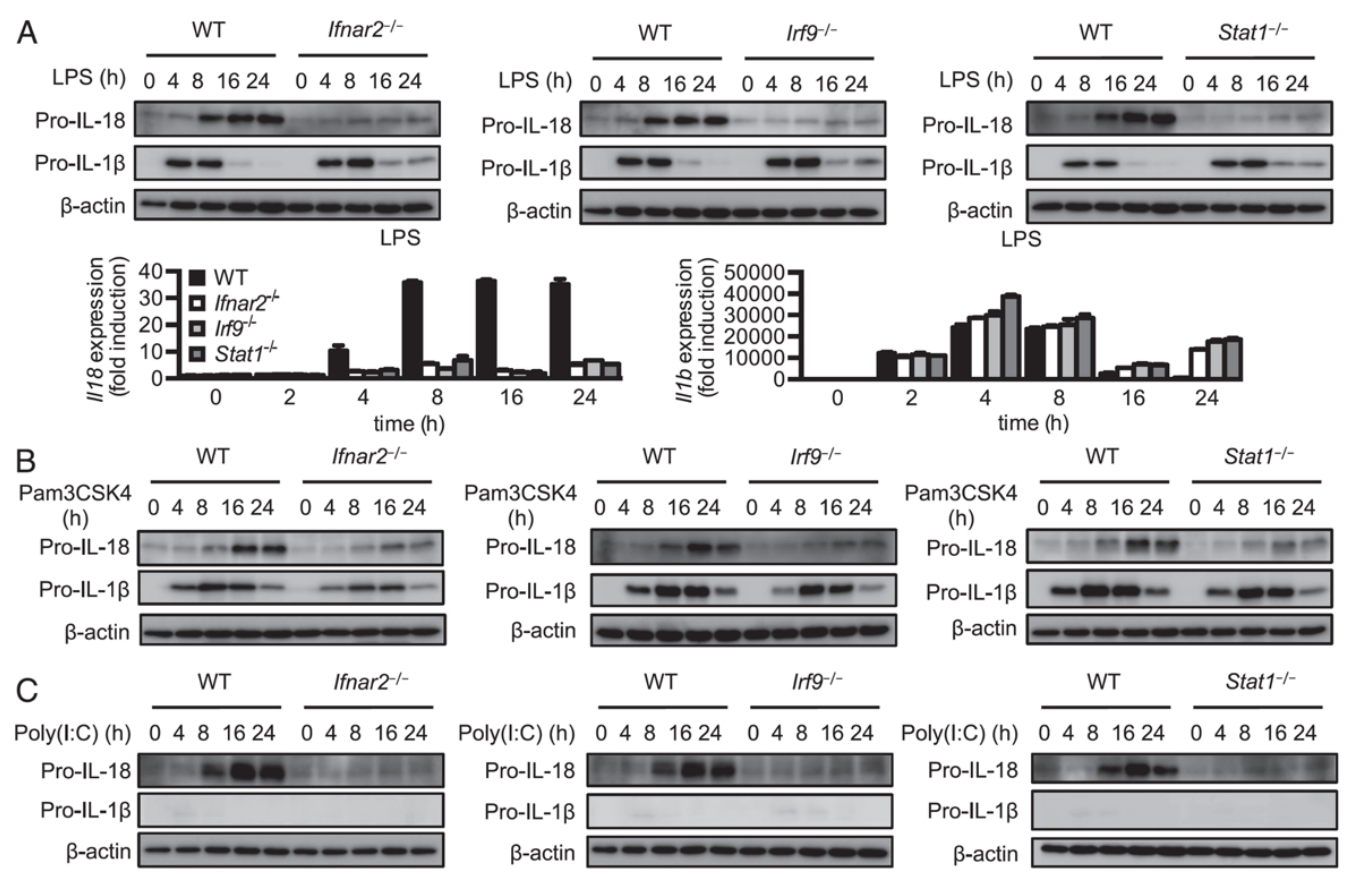

Figure 7-4. Components of type I IFN signaling are required for induction of IL18.

(A) Immunoblot analysis of pro-IL-18, pro-IL-1 $\beta$, and $\beta$-actin (loading control) and realtime qPCR analysis of genes encoding IL-18 and IL-1 $\beta$ in WT, Ifnar2 $2^{-/-}$, Irf $9^{-1-}$, or Stat $^{-1-}$ BMDMs at various time points after LPS treatment. (B and C) Immunoblot analysis of pro-IL-18, pro-IL-1 $\beta$, and $\beta$-actin (loading control) in WT, Ifnar $2^{-/-}$, Irf $f 9^{-/-}$, or Stat ${ }^{-1-}$ BMDMs at various time points after Pam3CSK4 (B) or poly(I:C) treatment (C). Data are representative of three independent experiments. 


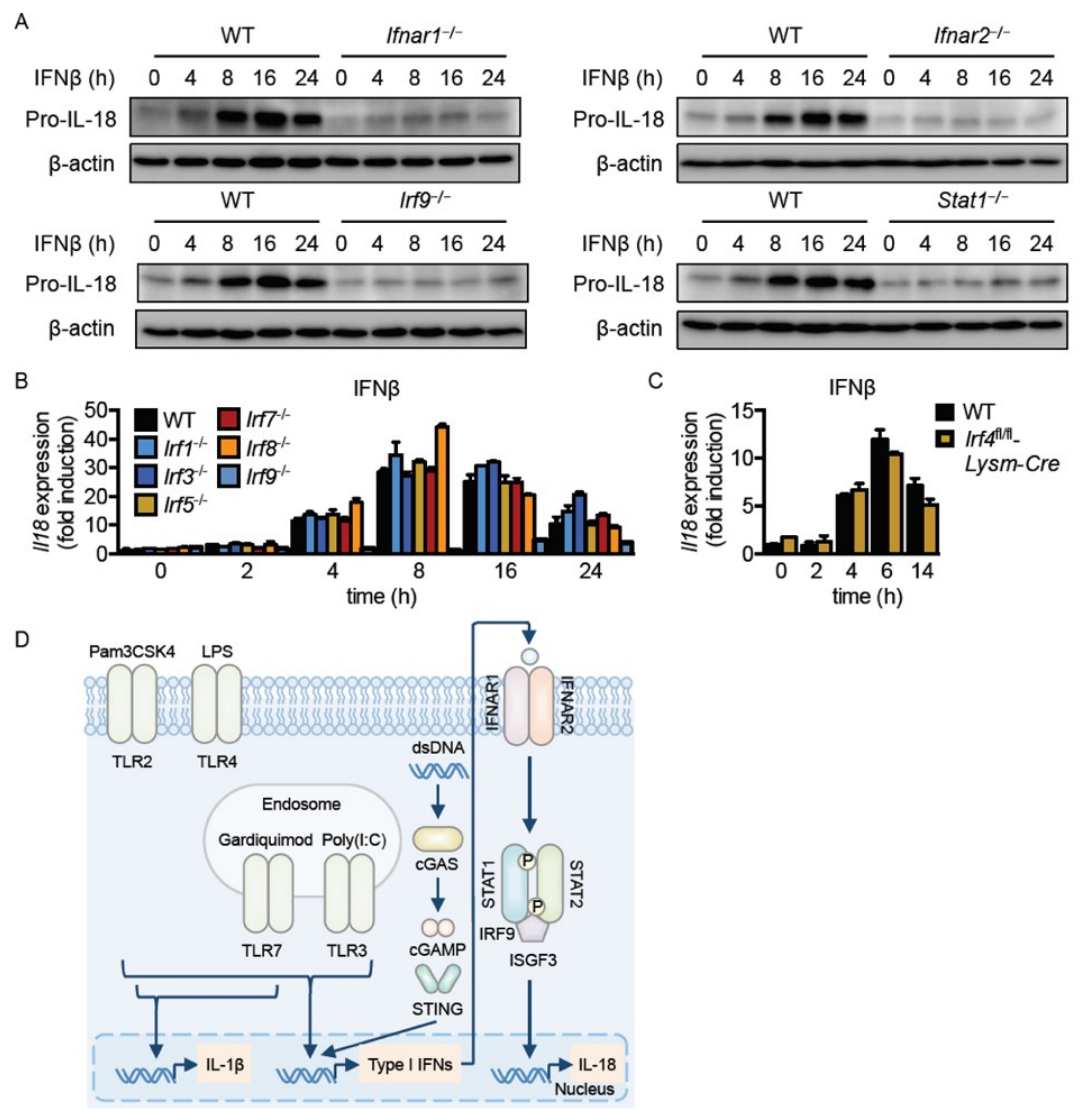

Figure 7-5. Type I IFNs induce IL-18 expression via ISGF3 and a model of differential regulation of pro-inflammatory cytokines IL-18 and IL-1ß.

(A) Immunoblot analysis of pro-IL-18 and $\beta$-actin (loading control) in WT, Ifnar $2^{-/}$, $\operatorname{Irf9^{-/-}}$ or Stat $^{-/-}$BMDMs at various times after treated with IFN $\beta$. (B) Real-time qPCR analysis of the gene encoding IL-18 in WT, $\operatorname{Irf1} 1^{-/}, \operatorname{Irf3^{-/-}}, \operatorname{Irf5} 5^{-/-}, \operatorname{Irf7^{-/}}$ or $\operatorname{Irf8^{-/-}}$ BMDMs at various times after treated with IFN $\beta$. (C) Real-time qPCR analysis of the gene encoding IL-18 in WT or Irf $4^{\mathrm{fl} / \mathrm{fl}}$-Lysm-Cre BMDMs at various times after treated with IFN $\beta$. (D) Expression of IL-18 is induced and sustained by stimulations of TLR2, TLR4, or TLR7 ligand, while IL-1 $\beta$ expression declines soon after reaching its peak level. The TLR3 and cGAS-STING pathways also upregulate IL-18 expression but are modest at inducing IL- $1 \beta$ expression. Importantly, type I IFN signaling downstream of TLRs and cGAS-STING pathway is crucial in mediating IL-18 induction. Data are representative of three independent experiments. 
degraded during chronic LPS stimulation [187]. This observation is in line with several studies showing that when macrophages are treated with TLR ligands, pro-IL-1 $\beta$ is sequestered in autophagosomes for degradation $[188,189]$. Of note, we found that although increases in mRNA levels were similar between $I l 18$ and $I l l b$ in response to Poly(I:C) in WT BMDMs, upregulation of IL- $1 \beta$ protein levels was much less robust compared with that of IL-18 levels (Fig. 1C). This could be resulted from decreased translational activity of $I l 1 b$ mRNA or lower stability of pro-IL-1 $\beta$ due to proteasome or autophagy-mediated degradation, revealing another layer of differential regulation between IL-18 and IL-1 $\beta$. In addition, the modest expression of IL-1 $\beta$ in response to Poly(I:C) is supported by a previous study showing that pro-IL-1 $\beta$ is much less induced by Poly(I:C) than by LPS or gardiquimod [190]. Our study also found that downregulation of both $I l 1 b$ mRNA and protein expression was ameliorated in cells lacking type I IFN signaling at later time points of LPS stimulation. An intriguing theory is that type I IFN signaling may be involved in the negative control of $I l 1 b$ expression. Indeed, macrophages lacking tyrosine kinase 2, which is downstream of the type I IFN receptor, have increased translation of $I l 1 b$ and hence high levels of pro-IL-1 $\beta$ in response to LPS [191]. Previous study has also demonstrated that type I IFN signaling suppresses IL-1 $\beta$ expression via IL-10-STAT3 signaling axis and inhibits proteolytic processing of IL-1 $\beta$ by NLRP3 inflammasome [192]. Interestingly, downregulation of IL-1 $\beta$ expression was not affected in BMDMs lacking type I IFN signaling compared with WT cells at later time points of Pam3CSK4 or gardiquimod stimulation (Fig. 2B, 2C, 3B and 3C). The MyD88 pathway might induce lower production of type I IFNs and consequently the negative control on IL-1 $\beta$ is less robust as compared to the TRIF pathway that is activated by LPS.

IL-1 family cytokines have pleiotropic functions and are involved in several inflammatory and autoinflammatory disease settings. Interestingly, IL- $1 \alpha$, IL-1 $\beta$ and IL18 have specific functions in regulating disease outcomes [36]. Autoinflammatory models associated with overt pyrin inflammasome activation are differentially mediated through IL-18 [193] or IL-1 $\beta$ [194] production. While levels of circulating IL-1 $\beta$ and IL18 in the serum are upregulated in patients bearing activating NLRC4 or NLRP3 mutation, IL-18 is induced to a greater extent in patients harboring mutation in NLRC4 [195]. IL-18 is also chronically elevated in several of the autoinflammatory diseases and inflammasomepathies [196]. However, the cause and effect of this chronically sustained elevated level of IL-18 is not studied well. Our current studies demonstrate that targeting type I IFN signaling pathway could be a way to regulate the pathologic level of IL-18 in the affected patients. In summary, our study demonstrated that during chronic stimulation, IL-18 expression is induced and sustained. In contrast, during chronic stimulations IL-1 $\beta$ is robustly induced but not sustained. Finally, we show that type I IFN signaling is necessary for the induction of IL-18, but not IL-1 $\beta$, which points to a critical and differential role for type I IFN in regulating IL-18 signaling. 


\section{CHAPTER 8. DISCUSSIONS}

Cytosolic nucleic acid sensors are pattern recognition receptors that mediate a range of physiological and pathological conditions. STING functions as an adaptor for cytosolic DNA sensing pathway induced by cGAS [10]. Our results demonstrated that STING is critical for protection against colon cancer by controlling overt colon inflammation, possibly through mediating activation of caspase-1 and casepase-1dependent IL-18 release. Shortly after our work was published [143], studies from other groups showed that expression of cGAS or STING is lost in colon carcinoma cell lines or gastric cancer [197, 198]. Moreover, tumor DNA has been identified to be recognized by cGAS-STING pathway in antigen-presenting cells leading to their activation, a process that requires production of type I IFNs induced by cGAS and STING [199, 200]. Activated antigen-presenting cells then induce potent anti-tumor $\mathrm{CD} 8^{+} \mathrm{T}$ cell responses. STING agonists have been further shown to provide anti-tumor responses in mouse models of melanoma, sarcoma, colon carcinoma, glioma, breast carcinoma, upper aerodigestive squamous cell carcinoma and pancreatic carcinoma [199-205]. The antitumorigenic properties of STING ligands in a variety of tumor types indicate a need to evaluate therapeutic values of STING agonists in the clinic. Indeed, small molecules that activate STING pathway are currently undergoing Phase I clinical trials to treat patients with solid tumors or lymphomas (NCT02675439 and NCT03172936). In addition, given that cGAMP has been recently shown to activate inflammasome in a STING-dependent manner in both mouse and human myeloid cells $[112,113]$, it is especially important to monitor inflammasome responses in those cancer patients administrated with STING ligands.

We have also demonstrated that the DNA sensor AIM2 plays a critical role in preventing against colon cancer induced by AOM and DSS treatment. Although AIM2 forms the inflammasome complex with ASC and caspase-1 upon its recognition of cytosolic dsDNA, we showed that inflammasome responses were intact in the absence of AIM2 during the tumor development, as indicated by similar activation level of caspase-1 and release of IL-18 and IL-1 $\beta$ in the colon and sera as WT mice. The finding showing an inflammasome-independent role for AIM2 in colon cancer is confirmed by a second study [206]. To further demonstrate this, it would be helpful to cross $\mathrm{Aim} 2^{-1-}$ mice with mice lacking other components such as $\mathrm{Caspl}^{-1-}$ mice, and compared tumor outcome between $\mathrm{Aim}^{-/-}$mice, $\mathrm{Casp}^{-/-}$mice and $\mathrm{Aim}^{-{ }^{-1}} \mathrm{Casp}^{-/-}$mice. The inflammasomeindependent role for AIM2 in regulating tumor development would suggest an additive effect of tumor suppression by AIM2 and caspase- 1 and that an enhanced tumor burden would be observed in Aim $2^{-1-} \mathrm{Caspl}^{-/-}$mice compared with Aim2 ${ }^{-/-}$mice or Casp 1 $1^{-/}$ mice following AOM and DSS treatment. The second study further identified that AIM2 interacts with DNA-dependent protein kinase (DNA-PK), a protein of PI3K-related family to restrict activation of AKT pathway downstream of the kinase in response to the growth factor, insulin-like growth factor-1 (IGF-1) [206]. It would be interesting to identify and generate AIM2 mutants with an impaired ability to form inflammasome induced by cytosolic dsDNA but being able to bind to DNA-PK to regulate AKT pathway. We have also discovered that AIM2 negatively controls intestinal stem cell 
activity by crossing $\mathrm{Aim} 2^{-/-}$with Prom $1^{\text {CreERT2-LacZ }(C-L) /+}$; Ctnnb ${ }^{\text {lox(ex3)/++}}$; RosaZsGreen mouse strain and observed increased generative capacity of stem cells in Aim2 $2^{-1-} ;$ Prom $^{\text {CreERT2-LacZ (C-L)/+ }} ;$ Ctnnb1 $1^{\text {lox(ex3)/+ }}$; RosaZsGreen compared with Aim2 $2^{+/+}$;Prom $1^{\text {CreERT2-LacZ }(C-L) /+} ;$ Ctnnb $1^{\text {lox }(\text { ex3 } 3) /+} ;$ RosaZs Green. To investigate the role of AIM2 in stem cell function more directly, it would be ideal to have $A i m 2^{\mathrm{fl} / \mathrm{fl}}$ mice crossed with Prom $1^{\text {CreERT2-LacZ }(C-L) /+} ;$ Ctnnb $1^{\text {lox(ex3)/+}} ;$ RosaZs Green mice to delete AIM2 in Prom $1^{+}$stem cell compartment and its progeny specifically.

AIM2 recognizes bacterial, viral or self-derived DNA in the cytosol and forms an inflammasome complex leading to maturation and release of IL-1 $\beta$ and IL-18 and pyroptotic cell death [1]. AIM2 inflammasome is critical for induction of immune responses when host encounters a variety of infectious agents including $F$. novicida. While type I IFN signaling is required for activating AIM2 inflammasome after $F$. novicida infection [1]. We found that AIM2 inflammasome activation is intact in mice lacking type I IFN signaling in response to F. novicida, suggesting that type I IFN signaling is not required for AIM2 inflammasome activation in vivo. We showed that components of type I IFN signaling pathway are detrimental to the host survival during F. novicida infection, which is consistent with a previous study using mice lacking one subunit of type I IFN receptor IFNAR1 [155]. Furthermore, the deleterious type I IFN signaling dominates against protective AIM2 inflammasome responses by activating TRAIL-mediated apoptotic cell death pathway. Types of cells that are responsible for producing type I IFNs and TRAIL during the infection are still remained to be discovered. Moreover, it is intriguing to identify which cell types undergo apoptosis in a type I IFN signaling-dependent manner leading to the susceptibility to $F$. novicida infection. We observed induction of apoptotic cell death in F. novicida-infected liver tissues, where the majority of cells are hepatocytes. It is likely that hepatocytes are targeted by type I IFNs to cell death in the liver during the infection. Apoptosis may also occur in immune cells including inflammatory monocytes and tissue-resident macrophages such as Kupffer cells, which have important roles in antimicrobial activities.

We further explored the role of GSDMD downstream of AIM2 inflammasome during $F$. novicida infection. Consistent with the susceptibility of mice lacking AIM2, ASC or caspase-1 [41, 43-45, 47, 83, 168], mice lacking GSDMD were also more sensitive to the infection. Interestingly, we further discovered that GSDMD is required for optimal caspase-1 activation in response to $F$. novicida, despite the fact that AIM2 inflammasome still mediates cleavage of GSDMD to release its functional N-terminal fragment. GSDMD has been generally considered as the executioner of pyroptosis, our findings advance current understanding of the function of GSDMD and suggest a role for GSDMD in regulating activation of AIM2 inflammasome. In addition to AIM2 inflammasome, GSDMD is cleaved by caspase-11 leading to activation of non-canonical NLRP3 inflammasome [52]. It is intriguing to hypothesize that GSDMD activates AIM2 inflammasome or non-canonical NLRP3 inflammasome by a shared mechanism via pore formation on the membrane of an unknown source. 
Our findings on distinctive mechanisms regulating the expression of inflammasome-associated cytokines IL-1 $\beta$ and IL-18 may provide explanations on several autoimmune diseases or infections where IL-1 $\beta$ and IL-18 have differential roles [65-67, 81, 173, 207, 208]. Given that induction of IL-18 is dependent on type I IFN signaling in BMDMs, effects of IL-18 should be taken into accounts in clinical therapies that modulate type I IFN signaling [209]. Consistent with our work, IL-18 expression is observed to be downregulated in Ifnar $1^{-1-}$ BMDMs in response to influenza virus [210]. Type I IFN signaling has also been shown to mediate IL-18 production by inflammatory monocytes during herpes simplex virus (HSV)-2 infection leading to the activation of natural killer (NK) cells [211], further highlighting the significance of our findings under pathological conditions. 


\section{LIST OF REFERENCES}

1. Man, S.M., R. Karki, and T.D. Kanneganti, AIM2 inflammasome in infection, cancer, and autoimmunity: Role in DNA sensing, inflammation, and innate immunity. Eur J Immunol, 2016. 46(2): p. 269-80.

2. Kato, H., et al., Length-dependent recognition of double-stranded ribonucleic acids by retinoic acid-inducible gene-I and melanoma differentiation-associated gene 5. J Exp Med, 2008. 205(7): p. 1601-10.

3. Hornung, V., et al., 5'-Triphosphate RNA is the ligand for RIG-I. Science, 2006. 314(5801): p. 994-7.

4. Pichlmair, A., et al., RIG-I-mediated antiviral responses to single-stranded RNA bearing 5'-phosphates. Science, 2006. 314(5801): p. 997-1001.

5. Goubau, D., et al., Antiviral immunity via RIG-I-mediated recognition of $R N A$ bearing 5'-diphosphates. Nature, 2014. 514(7522): p. 372-375.

6. Kawai, T., et al., IPS-1, an adaptor triggering RIG-I-and Mda5-mediated type I interferon induction. Nat Immunol, 2005. 6(10): p. 981-8.

7. Meylan, E., et al., Cardif is an adaptor protein in the RIG-I antiviral pathway and is targeted by hepatitis $C$ virus. Nature, 2005. 437(7062): p. 1167-72.

8. Seth, R.B., et al., Identification and characterization of MAVS, a mitochondrial antiviral signaling protein that activates NF-kappaB and IRF 3. Cell, 2005. 122(5): p. 669-82.

9. $\mathrm{Xu}, \mathrm{L} . \mathrm{G}$., et al., VISA is an adapter protein required for virus-triggered IFN-beta signaling. Mol Cell, 2005. 19(6): p. 727-40.

10. $\mathrm{Wu}, \mathrm{J}$. and Z.J. Chen, Innate immune sensing and signaling of cytosolic nucleic acids. Annu Rev Immunol, 2014. 32: p. 461-88.

11. Kato, H., et al., Differential roles of MDA5 and RIG-I helicases in the recognition of RNA viruses. Nature, 2006. 441(7089): p. 101-5.

12. Sun, L., et al., Cyclic GMP-AMP synthase is a cytosolic DNA sensor that activates the type I interferon pathway. Science, 2013. 339(6121): p. 786-91.

13. Wu, J., et al., Cyclic GMP-AMP is an endogenous second messenger in innate immune signaling by cytosolic DNA. Science, 2013. 339(6121): p. 826-30.

14. Zhong, B., et al., The adaptor protein MITA links virus-sensing receptors to IRF3 transcription factor activation. Immunity, 2008. 29(4): p. 538-50.

15. Ishikawa, H. and G.N. Barber, STING is an endoplasmic reticulum adaptor that facilitates innate immune signalling. Nature, 2008. 455(7213): p. 674-8.

16. Sun, W., et al., ERIS, an endoplasmic reticulum IFN stimulator, activates innate immune signaling through dimerization. Proc Natl Acad Sci U S A, 2009.

106(21): p. 8653-8.

17. Burdette, D.L., et al., STING is a direct innate immune sensor of cyclic di-GMP. Nature, 2011. 478(7370): p. 515-8.

18. Saitoh, T., et al., Atg9a controls dsDNA-driven dynamic translocation of STING and the innate immune response. Proc Natl Acad Sci U S A, 2009. 106(49): p. 20842-6. 
19. Ishikawa, H., Z. Ma, and G.N. Barber, STING regulates intracellular DNAmediated, type I interferon-dependent innate immunity. Nature, 2009. 461(7265): p. 788-92.

20. Tanaka, Y. and Z.J. Chen, STING specifies IRF3 phosphorylation by TBK1 in the cytosolic DNA signaling pathway. Sci Signal, 2012. 5(214): p. ra20.

21. Marinho, F.V., et al., The Emerging Roles of STING in Bacterial Infections. Trends Microbiol, 2017. 25(11): p. 906-918.

22. Woodward, J.J., A.T. Iavarone, and D.A. Portnoy, c-di-AMP secreted by intracellular Listeria monocytogenes activates a host type I interferon response. Science, 2010. 328(5986): p. 1703-5.

23. Roulois, D., et al., DNA-Demethylating Agents Target Colorectal Cancer Cells by Inducing Viral Mimicry by Endogenous Transcripts. Cell, 2015. 162(5): p. 96173.

24. Ferlay, J., et al., Cancer incidence and mortality worldwide: sources, methods and major patterns in GLOBOCAN 2012. Int J Cancer, 2015. 136(5): p. E359-86.

25. Arnold, M., et al., Global patterns and trends in colorectal cancer incidence and mortality. Gut, 2017. 66(4): p. 683-691.

26. Dihlmann, S., et al., Lack of Absent in Melanoma 2 (AIM2) expression in tumor cells is closely associated with poor survival in colorectal cancer patients. Int $\mathrm{J}$ Cancer, 2014. 135(10): p. 2387-96.

27. Allen, I.C., et al., The NLRP3 inflammasome functions as a negative regulator of tumorigenesis during colitis-associated cancer. J Exp Med, 2010. 207(5): p. 1045-56.

28. Zaki, M.H., et al., The NLRP3 inflammasome protects against loss of epithelial integrity and mortality during experimental colitis. Immunity, 2010. 32(3): p. 379-91.

29. Zaki, M.H., et al., IL-18 production downstream of the Nlrp3 inflammasome confers protection against colorectal tumor formation. J Immunol, 2010. 185(8): p. 4912-20.

30. Allen, I.C., et al., NLRP12 suppresses colon inflammation and tumorigenesis through the negative regulation of noncanonical NF-kappaB signaling. Immunity, 2012. 36(5): p. 742-54.

31. Zaki, M.H., et al., The NOD-like receptor NLRP12 attenuates colon inflammation and tumorigenesis. Cancer Cell, 2011. 20(5): p. 649-60.

32. Burckstummer, T., et al., An orthogonal proteomic-genomic screen identifies AIM2 as a cytoplasmic DNA sensor for the inflammasome. Nat Immunol, 2009. 10(3): p. 266-72.

33. Fernandes-Alnemri, T., et al., AIM2 activates the inflammasome and cell death in response to cytoplasmic DNA. Nature, 2009. 458(7237): p. 509-13.

34. Hornung, V., et al., AIM2 recognizes cytosolic dsDNA and forms a caspase-1activating inflammasome with ASC. Nature, 2009. 458(7237): p. 514-8.

35. Roberts, T.L., et al., HIN-200 proteins regulate caspase activation in response to foreign cytoplasmic DNA. Science, 2009. 323(5917): p. 1057-60.

36. Man, S.M. and T.D. Kanneganti, Converging roles of caspases in inflammasome activation, cell death and innate immunity. Nat Rev Immunol, 2016. 16(1): p. 721. 
37. DeYoung, K.L., et al., Cloning a novel member of the human interferon-inducible gene family associated with control of tumorigenicity in a model of human melanoma. Oncogene, 1997. 15(4): p. 453-7.

38. Woerner, S.M., et al., The putative tumor suppressor AIM2 is frequently affected by different genetic alterations in microsatellite unstable colon cancers. Genes Chromosomes Cancer, 2007. 46(12): p. 1080-9.

39. Kim, T.M., P.W. Laird, and P.J. Park, The landscape of microsatellite instability in colorectal and endometrial cancer genomes. Cell, 2013. 155(4): p. 858-68.

40. Dihlmann, S., et al., Lack of Absent in Melanoma 2 (AIM2) expression in tumor cells is closely associated with poor survival in colorectal cancer patients. Int J Cancer, 2014.

41. Fernandes-Alnemri, T., et al., The AIM2 inflammasome is critical for innate immunity to Francisella tularensis. Nat Immunol, 2010. 11(5): p. 385-93.

42. Rathinam, V.A., et al., The AIM2 inflammasome is essential for host defense against cytosolic bacteria and DNA viruses. Nat Immunol, 2010. 11(5): p. 395402.

43. Jones, J.W., et al., Absent in melanoma 2 is required for innate immune recognition of Francisella tularensis. Proc Natl Acad Sci U S A, 2010. 107(21): p. 9771-6.

44. Man, S.M., et al., The transcription factor IRF1 and guanylate-binding proteins target activation of the AIM2 inflammasome by Francisella infection. Nat Immunol, 2015. 16(5): p. 467-75.

45. Meunier, E., et al., Guanylate-binding proteins promote activation of the AIM2 inflammasome during infection with Francisella novicida. Nat Immunol, 2015. 16(5): p. 476-84.

46. Storek, K.M., et al., cGAS and Ifi204 cooperate to produce type I IFNs in response to Francisella infection. J Immunol, 2015. 194(7): p. 3236-45.

47. Man, S.M., et al., IRGB10 Liberates Bacterial Ligands for Sensing by the AIM2 and Caspase-11-NLRP3 Inflammasomes. Cell, 2016. 167(2): p. 382-396 e17.

48. Kim, B.H., et al., IFN-inducible GTPases in host cell defense. Cell Host Microbe, 2012. 12(4): p. 432-44.

49. Kovarik, P., et al., Type I Interferons in Bacterial Infections: A Balancing Act. Front Immunol, 2016. 7: p. 652.

50. McNab, F., et al., Type I interferons in infectious disease. Nat Rev Immunol, 2015. 15(2): p. 87-103.

51. He, W.T., et al., Gasdermin D is an executor of pyroptosis and required for interleukin-1beta secretion. Cell Res, 2015. 25(12): p. 1285-98.

52. Kayagaki, N., et al., Caspase-11 cleaves gasdermin D for non-canonical inflammasome signalling. Nature, 2015. 526(7575): p. 666-71.

53. Shi, J., et al., Cleavage of GSDMD by inflammatory caspases determines pyroptotic cell death. Nature, 2015. 526(7575): p. 660-5.

54. Chen, X., et al., Pyroptosis is driven by non-selective gasdermin-D pore and its morphology is different from MLKL channel-mediated necroptosis. Cell Res, 2016. 26(9): p. 1007-20.

55. Sborgi, L., et al., GSDMD membrane pore formation constitutes the mechanism of pyroptotic cell death. EMBO J, 2016. 35(16): p. 1766-78. 
56. Ding, J., et al., Pore-forming activity and structural autoinhibition of the gasdermin family. Nature, 2016. 535(7610): p. 111-6.

57. Liu, X., et al., Inflammasome-activated gasdermin D causes pyroptosis by forming membrane pores. Nature, 2016. 535(7610): p. 153-8.

58. Aglietti, R.A., et al., GsdmD p30 elicited by caspase-11 during pyroptosis forms pores in membranes. Proc Natl Acad Sci U S A, 2016. 113(28): p. 7858-63.

59. Heilig, R., et al., The Gasdermin-D pore acts as a conduit for IL-1beta secretion in mice. Eur J Immunol, 2017.

60. Evavold, C.L., et al., The Pore-Forming Protein Gasdermin D Regulates Interleukin-1 Secretion from Living Macrophages. Immunity, 2018. 48(1): p. 3544 e6.

61. del Barrio, L., et al., Production of anti-LPS IgM by Bla B cells depends on ILlbeta and is protective against lung infection with Francisella tularensis LVS. PLoS Pathog, 2015. 11(3): p. e1004706.

62. Periasamy, S., et al., Inflammasome-Independent NLRP3 Restriction of a Protective Early Neutrophil Response to Pulmonary Tularemia. PLoS Pathog, 2016. 12(12): p. e1006059.

63. Broz, P. and V.M. Dixit, Inflammasomes: mechanism of assembly, regulation and signalling. Nat Rev Immunol, 2016. 16(7): p. 407-20.

64. Kupz, A., et al., NLRC4 inflammasomes in dendritic cells regulate noncognate effector function by memory CD8(+) T cells. Nat Immunol, 2012. 13(2): p. 162-9.

65. Dinarello, C.A., Immunological and inflammatory functions of the interleukin-1 family. Annu Rev Immunol, 2009. 27: p. 519-50.

66. Garlanda, C., C.A. Dinarello, and A. Mantovani, The interleukin-1 family: back to the future. Immunity, 2013. 39(6): p. 1003-18.

67. Sims, J.E. and D.E. Smith, The IL-1 family: regulators of immunity. Nat Rev Immunol, 2010. 10(2): p. 89-102.

68. Puren, A.J., G. Fantuzzi, and C.A. Dinarello, Gene expression, synthesis, and secretion of interleukin 18 and interleukin 1beta are differentially regulated in human blood mononuclear cells and mouse spleen cells. Proc Natl Acad Sci U S A, 1999. 96(5): p. 2256-61.

69. Marshall, J.D., et al., Regulation of human IL-18 mRNA expression. Clin Immunol, 1999. 90(1): p. 15-21.

70. Bohle, B., et al., Oligodeoxynucleotides containing CpG motifs induce IL-12, IL18 and IFN-gamma production in cells from allergic individuals and inhibit IgE synthesis in vitro. Eur J Immunol, 1999. 29(7): p. 2344-53.

71. Pirhonen, J., et al., Virus infection activates IL-1 beta and IL-18 production in human macrophages by a caspase-1-dependent pathway. J Immunol, 1999. 162(12): p. 7322-9.

72. Sauer, J.D., et al., The N-ethyl-N-nitrosourea-induced Goldenticket mouse mutant reveals an essential function of Sting in the in vivo interferon response to Listeria monocytogenes and cyclic dinucleotides. Infect Immun, 2011. 79(2): p. 688-94.

73. Mariathasan, S., et al., Differential activation of the inflammasome by caspase-1 adaptors ASC and Ipaf. Nature, 2004. 430(6996): p. 213-8.

74. Kayagaki, N., et al., Non-canonical inflammasome activation targets caspase-11. Nature, 2011. 479(7371): p. 117-21. 
75. Kuida, K., et al., Decreased apoptosis in the brain and premature lethality in CPP32-deficient mice. Nature, 1996. 384(6607): p. 368-72.

76. Lakhani, S.A., et al., Caspases 3 and 7: key mediators of mitochondrial events of apoptosis. Science, 2006. 311(5762): p. 847-51.

77. Zhu, L., et al., Prominin 1 marks intestinal stem cells that are susceptible to neoplastic transformation. Nature, 2009. 457(7229): p. 603-7.

78. Muller, U., et al., Functional role of type I and type II interferons in antiviral defense. Science, 1994. 264(5167): p. 1918-21.

79. Fenner, J.E., et al., Suppressor of cytokine signaling 1 regulates the immune response to infection by a unique inhibition of type I interferon activity. Nat Immunol, 2006. 7(1): p. 33-9.

80. Sato, M., et al., Distinct and essential roles of transcription factors IRF-3 and IRF-7 in response to viruses for IFN-alpha/beta gene induction. Immunity, 2000. 13(4): p. 539-48.

81. Zhu, Q. and T.D. Kanneganti, Cutting Edge: Distinct Regulatory Mechanisms Control Proinflammatory Cytokines IL-18 and IL-1beta. J Immunol, 2017. 198(11): p. 4210-4215.

82. Honda, K., et al., IRF-7 is the master regulator of type-I interferon-dependent immune responses. Nature, 2005. 434(7034): p. 772-7.

83. Zhu, Q., et al., Detrimental Type I Interferon Signaling Dominates Protective AIM2 Inflammasome Responses during Francisella novicida Infection. Cell Rep, 2018. 22(12): p. 3168-3174.

84. Kimura, T., et al., Essential and non-redundant roles of 448 (ISGF3 gamma) and $I R F-1$ in both type I and type II interferon responses, as revealed by gene targeting studies. Genes Cells, 1996. 1(1): p. 115-24.

85. Karki, R., et al., IRF8 Regulates Transcription of $<e m>$ Naip $</ e m>$ s for NLRC4 Inflammasome Activation. Cell, 2018. 173(4): p. 920-933.e13.

86. Man S.M., et al., Critical Role for the DNA Sensor AIM2 in Stem Cell Proliferation and Cancer. Cell, 2015. 162(1): p. 45-58.

87. Daley-Bauer, L.P., et al., Mouse cytomegalovirus M36 and M45 death suppressors cooperate to prevent inflammation resulting from antiviral programmed cell death pathways. Proc Natl Acad Sci U S A, 2017. 114(13): p. E2786-E2795.

88. Yui, S., et al., Functional engraftment of colon epithelium expanded in vitro from a single adult Lgr5(+) stem cell. Nat Med, 2012. 18(4): p. 618-23.

89. Willert, K., et al., Wnt proteins are lipid-modified and can act as stem cell growth factors. Nature, 2003. 423(6938): p. 448-52.

90. DeSantis, T.Z., et al., Greengenes, a chimera-checked 16S rRNA gene database and workbench compatible with ARB. Appl Environ Microbiol, 2006. 72(7): p. 5069-72.

91. Slater, G.S. and E. Birney, Automated generation of heuristics for biological sequence comparison. BMC Bioinformatics, 2005. 6: p. 31.

92. Edgar, R.C., Search and clustering orders of magnitude faster than BLAST. Bioinformatics, 2010. 26(19): p. 2460-1.

93. Lee, B.L., et al., ASC-and caspase-8-dependent apoptotic pathway diverges from the NLRC4 inflammasome in macrophages. Sci Rep, 2018. 8(1): p. 3788. 
94. Qi, X., et al., Critical role of caspase-8-mediated IL-1 signaling in promoting Th2 responses during asthma pathogenesis. Mucosal Immunol, 2017. 10(1): p. 128138.

95. Siegel, R.L., K.D. Miller, and A. Jemal, Cancer statistics, 2018. CA Cancer J Clin, 2018. 68(1): p. 7-30.

96. Chen, G.Y. and G. Nunez, Inflammasomes in intestinal inflammation and cancer. Gastroenterology, 2011. 141(6): p. 1986-99.

97. Elinav, E., et al., Inflammation-induced cancer: crosstalk between tumours, immune cells and microorganisms. Nat Rev Cancer, 2013. 13(11): p. 759-71.

98. Hornung, V., et al., OAS proteins and cGAS: unifying concepts in sensing and responding to cytosolic nucleic acids. Nat Rev Immunol, 2014. 14(8): p. 521-8.

99. Unterholzner, L., et al., IFI16 is an innate immune sensor for intracellular DNA. Nat Immunol, 2010. 11(11): p. 997-1004.

100. Zhang, Z., et al., The helicase DDX41 senses intracellular DNA mediated by the adaptor STING in dendritic cells. Nat Immunol, 2011. 12(10): p. 959-65.

101. Burdette, D.L. and R.E. Vance, STING and the innate immune response to nucleic acids in the cytosol. Nat Immunol, 2013. 14(1): p. 19-26.

102. Sander, L.E., et al., Detection of prokaryotic $m R N A$ signifies microbial viability and promotes immunity. Nature, 2011. 474(7351): p. 385-9.

103. Kanneganti, T.D., et al., Bacterial RNA and small antiviral compounds activate caspase-1 through cryopyrin/Nalp3. Nature, 2006. 440(7081): p. 233-6.

104. Kailasan Vanaja, S., et al., Bacterial RNA:DNA hybrids are activators of the NLRP3 inflammasome. Proc Natl Acad Sci U S A, 2014. 111(21): p. 7765-70.

105. Hou, J., et al., Hepatic RIG-I predicts survival and interferon-alpha therapeutic response in hepatocellular carcinoma. Cancer Cell, 2014. 25(1): p. 49-63.

106. Widau, R.C., et al., RIG-I-like receptor LGP2 protects tumor cells from ionizing radiation. Proc Natl Acad Sci U S A, 2014. 111(4): p. E484-91.

107. Puebla-Osorio, N., et al., A novel Ku70 function in colorectal homeostasis separate from nonhomologous end joining. Oncogene, 2014. 33(21): p. 2748-57.

108. Bollrath, J., et al., gp130-mediated Stat3 activation in enterocytes regulates cell survival and cell-cycle progression during colitis-associated tumorigenesis. Cancer Cell, 2009. 15(2): p. 91-102.

109. Grivennikov, S., et al., IL-6 and Stat3 are required for survival of intestinal epithelial cells and development of colitis-associated cancer. Cancer Cell, 2009. 15(2): p. 103-13.

110. Yu, H., D. Pardoll, and R. Jove, STATs in cancer inflammation and immunity: a leading role for STAT3. Nat Rev Cancer, 2009. 9(11): p. 798-809.

111. Sharma, D., et al., Pyrin Inflammasome Regulates Tight Junction Integrity to Restrict Colitis and Tumorigenesis. Gastroenterology, 2018. 154(4): p. 948-964 e8.

112. Gaidt, M.M., et al., The DNA Inflammasome in Human Myeloid Cells Is Initiated by a STING-Cell Death Program Upstream of NLRP3. Cell, 2017. 171(5): p. 1110-1124 e18.

113. Swanson, K.V., et al., A noncanonical function of cGAMP in inflammasome priming and activation. J Exp Med, 2017. 214(12): p. 3611-3626. 
114. Ng, K.W., et al., cGAS-STING and Cancer: Dichotomous Roles in Tumor Immunity and Development. Trends Immunol, 2018. 39(1): p. 44-54.

115. Ahn, J., et al., Extrinsic Phagocyte-Dependent STING Signaling Dictates the Immunogenicity of Dying Cells. Cancer Cell, 2018.

116. Gehrke, N., et al., Oxidative damage of DNA confers resistance to cytosolic nuclease TREX1 degradation and potentiates STING-dependent immune sensing. Immunity, 2013. 39(3): p. 482-95.

117. Westbrook, A.M. and R.H. Schiestl, Atm-deficient mice exhibit increased sensitivity to dextran sulfate sodium-induced colitis characterized by elevated DNA damage and persistent immune activation. Cancer Res, 2010. 70(5): $\mathrm{p}$. 1875-84.

118. Ramalingam, S.S., T.K. Owonikoko, and F.R. Khuri, Lung cancer: New biological insights and recent therapeutic advances. CA Cancer J Clin, 2011. 61(2): p. 91-112.

119. Schulmann, K., et al., HNPCC-associated small bowel cancer: clinical and molecular characteristics. Gastroenterology, 2005. 128(3): p. 590-9.

120. Jin, T., et al., Structures of the HIN domain:DNA complexes reveal ligand binding and activation mechanisms of the AIM2 inflammasome and IFI16 receptor. Immunity, 2012. 36(4): p. 561-71.

121. Dombrowski, Y., et al., Cytosolic DNA triggers inflammasome activation in keratinocytes in psoriatic lesions. Sci Transl Med, 2011. 3(82): p. 82ra38.

122. Schulmann, K., et al., HNPCC-associated small bowel cancer: clinical and molecular characteristics. Gastroenterology, 2005. 128(3): p. 590-599.

123. Kim, T.-M., P. Laird, and P. Park, The landscape of microsatellite instability in colorectal and endometrial cancer genomes. Cell, 2013. 155(4): p. 858-868.

124. Woerner, S., et al., The putative tumor suppressor AIM2 is frequently affected by different genetic alterations in microsatellite unstable colon cancers. Genes, chromosomes \& cancer, 2007. 46(12): p. 1080-1089.

125. Dihlmann, S., et al., Lack of Absent in Melanoma 2 (AIM2) expression in tumor cells is closely associated with poor survival in colorectal cancer patients. International journal of cancer. Journal international du cancer, 2014.

126. Zhao, L., et al., Comparative proteomic analysis identifies proteins associated with the development and progression of colorectal carcinoma. FEBS J, 2010. 277(20): p. 4195-204.

127. Vonlanthen, J., et al., A comprehensive look at transcription factor gene expression changes in colorectal adenomas. BMC Cancer, 2014. 14: p. 46.

128. Bonte, D., et al., Cdc7-Dbf4 kinase overexpression in multiple cancers and tumor cell lines is correlated with p53 inactivation. Neoplasia, 2008. 10(9): p. 920-31.

129. Vivanco, I. and C.L. Sawyers, The phosphatidylinositol 3-Kinase AKT pathway in human cancer. Nat Rev Cancer, 2002. 2(7): p. 489-501.

130. Stambolic, V., et al., Negative regulation of PKB/Akt-dependent cell survival by the tumor suppressor PTEN. Cell, 1998. 95(1): p. 29-39.

131. Vazquez, F., et al., Phosphorylation of the PTEN tail regulates protein stability and function. Mol Cell Biol, 2000. 20(14): p. 5010-8.

132. Barker, N., et al., Crypt stem cells as the cells-of-origin of intestinal cancer. Nature, 2009. 457(7229): p. 608-11. 
133. Snippert, H.J., et al., Prominin-1/CD133 marks stem cells and early progenitors in mouse small intestine. Gastroenterology, 2009. 136(7): p. 2187-2194 e1.

134. Bienz, M. and H. Clevers, Linking colorectal cancer to Wnt signaling. Cell, 2000. 103(2): p. 311-20.

135. Sparks, A.B., et al., Mutational analysis of the APC/beta-catenin/Tcf pathway in colorectal cancer. Cancer Res, 1998. 58(6): p. 1130-4.

136. Elinav, E., et al., NLRP6 inflammasome regulates colonic microbial ecology and risk for colitis. Cell, 2011. 145(5): p. 745-757.

137. Normand, S., et al., Nod-like receptor pyrin domain-containing protein 6 (NLRP6) controls epithelial self-renewal and colorectal carcinogenesis upon injury. Proceedings of the National Academy of Sciences of the United States of America, 2011. 108(23): p. 9601-9606.

138. Zackular, J.P., et al., The gut microbiome modulates colon tumorigenesis. MBio, 2013. 4(6): p. e00692-13.

139. Watanabe, T., et al., Muramyl dipeptide activation of nucleotide-binding oligomerization domain 2 protects mice from experimental colitis. J Clin Invest, 2008. 118(2): p. 545-59.

140. $\mathrm{Hu}, \mathrm{B}$., et al., Inflammation-induced tumorigenesis in the colon is regulated by caspase-1 and NLRC4. Proc Natl Acad Sci U S A, 2010. 107(50): p. 21635-40.

141. Elinav, E., et al., NLRP6 inflammasome regulates colonic microbial ecology and risk for colitis. Cell, 2011. 145(5): p. 745-57.

142. Normand, S., et al., Nod-like receptor pyrin domain-containing protein 6 (NLRP6) controls epithelial self-renewal and colorectal carcinogenesis upon injury. Proc Natl Acad Sci U S A, 2011. 108(23): p. 9601-6.

143. Zhu, Q., et al., Cutting edge: STING mediates protection against colorectal tumorigenesis by governing the magnitude of intestinal inflammation. J Immunol, 2014. 193(10): p. 4779-82.

144. Man, S.M. and T.D. Kanneganti, Regulation of inflammasome activation. Immunol Rev, 2015. 265(1): p. 6-21.

145. Wlodarska, M., et al., NLRP6 inflammasome orchestrates the colonic hostmicrobial interface by regulating goblet cell mucus secretion. Cell, 2014. 156(5): p. 1045-59.

146. Patsos, G., et al., Restoration of absent in melanoma 2 (AIM2) induces G2/M cell cycle arrest and promotes invasion of colorectal cancer cells. Int J Cancer, 2010. 126(8): p. 1838-49.

147. Okada, T., et al., Microbiota-derived lactate accelerates colon epithelial cell turnover in starvation-refed mice. Nat Commun, 2013. 4: p. 1654.

148. Song, X., et al., Alterations in the microbiota drive interleukin-17C production from intestinal epithelial cells to promote tumorigenesis. Immunity, 2014. 40(1): p. 140-52.

149. Belcheva, A., et al., Gut microbial metabolism drives transformation of MSH2deficient colon epithelial cells. Cell, 2014. 158(2): p. 288-99.

150. Man, S.M., R. Karki, and T.D. Kanneganti, Molecular mechanisms and functions of pyroptosis, inflammatory caspases and inflammasomes in infectious diseases. Immunol Rev, 2017. 277(1): p. 61-75. 
151. Henry, T., et al., Type I interferon signaling is required for activation of the inflammasome during Francisella infection. J Exp Med, 2007. 204(5): p. 987-94.

152. Cole, L.E., et al., Macrophage proinflammatory response to Francisella tularensis live vaccine strain requires coordination of multiple signaling pathways. J Immunol, 2008. 180(10): p. 6885-91.

153. Malireddi, R.K. and T.D. Kanneganti, Role of type I interferons in inflammasome activation, cell death, and disease during microbial infection. Front Cell Infect Microbiol, 2013. 3: p. 77.

154. de Weerd, N.A., et al., Structural basis of a unique interferon-beta signaling axis mediated via the receptor IFNAR1. Nat Immunol, 2013. 14(9): p. 901-7.

155. Henry, T., et al., Type I IFN signaling constrains $I L-17 A / F$ secretion by gammadelta $T$ cells during bacterial infections. J Immunol, 2010. 184(7): p. 3755-67.

156. Castiglia, V., et al., Type I Interferon Signaling Prevents IL-1beta-Driven Lethal Systemic Hyperinflammation during Invasive Bacterial Infection of Soft Tissue. Cell Host Microbe, 2016. 19(3): p. 375-87.

157. Corrales, L., et al., Antagonism of the STING Pathway via Activation of the AIM2 Inflammasome by Intracellular DNA. J Immunol, 2016. 196(7): p. 3191-8.

158. Lamkanfi, M., et al., Targeted peptidecentric proteomics reveals caspase-7 as a substrate of the caspase-1 inflammasomes. Mol Cell Proteomics, 2008. 7(12): p. 2350-63.

159. Carrero, J.A., B. Calderon, and E.R. Unanue, Type I interferon sensitizes lymphocytes to apoptosis and reduces resistance to Listeria infection. J Exp Med, 2004. 200(4): p. 535-40.

160. de Almeida, L.A., et al., MyD88 and STING signaling pathways are required for IRF3-mediated IFN-beta induction in response to Brucella abortus infection. PLoS One, 2011. 6(8): p. e23135.

161. O'Connell, R.M., et al., Type I interferon production enhances susceptibility to Listeria monocytogenes infection. J Exp Med, 2004. 200(4): p. 437-45.

162. Carrero, J.A., B. Calderon, and E.R. Unanue, Lymphocytes are detrimental during the early innate immune response against Listeria monocytogenes. J Exp Med, 2006. 203(4): p. 933-40.

163. Zheng, S.J., et al., Reduced apoptosis and ameliorated listeriosis in TRAIL-null mice. J Immunol, 2004. 173(9): p. 5652-8.

164. Zhou, Z., M.J. Xu, and B. Gao, Hepatocytes: a key cell type for innate immunity. Cell Mol Immunol, 2016. 13(3): p. 301-15.

165. Muruve, D.A., et al., The inflammasome recognizes cytosolic microbial and host DNA and triggers an innate immune response. Nature, 2008. 452(7183): p. 103-7.

166. Baum, R., et al., Cutting edge: AIM2 and endosomal TLRs differentially regulate arthritis and autoantibody production in DNase II-deficient mice. J Immunol, 2015. 194(3): p. 873-7.

167. Jakobs, C., S. Perner, and V. Hornung, AIM2 Drives Joint Inflammation in a SelfDNA Triggered Model of Chronic Polyarthritis. PLoS One, 2015. 10(6): p. e0131702.

168. Mariathasan, S., et al., Innate immunity against Francisella tularensis is dependent on the ASC/caspase-1 axis. J Exp Med, 2005. 202(8): p. 1043-9. 
169. Schneider, K.S., et al., The Inflammasome Drives GSDMD-Independent Secondary Pyroptosis and IL-1 Release in the Absence of Caspase-1 Protease Activity. Cell Rep, 2017. 21(13): p. 3846-3859.

170. Wang, X., et al., Structure and biosynthesis of free lipid A molecules that replace lipopolysaccharide in Francisella tularensis subsp. novicida. Biochemistry, 2006. 45(48): p. 14427-40.

171. Rogers, C., et al., Cleavage of DFNA5 by caspase-3 during apoptosis mediates progression to secondary necrotic/pyroptotic cell death. Nat Commun, 2017. 8: p. 14128.

172. Wang, Y., et al., Chemotherapy drugs induce pyroptosis through caspase-3 cleavage of a gasdermin. Nature, 2017. 547(7661): p. 99-103.

173. Dinarello, C.A., A. Simon, and J.W. van der Meer, Treating inflammation by blocking interleukin-1 in a broad spectrum of diseases. Nat Rev Drug Discov, 2012. 11(8): p. 633-52.

174. Pizarro, T.T., et al., IL-18, a novel immunoregulatory cytokine, is up-regulated in Crohn's disease: expression and localization in intestinal mucosal cells. J Immunol, 1999. 162(11): p. 6829-35.

175. Stoll, S., et al., Production of functional IL-18 by different subtypes of murine and human dendritic cells (DC): DC-derived IL-18 enhances IL-12-dependent ThI development. Eur J Immunol, 1998. 28(10): p. 3231-9.

176. Matsui, K., et al., Propionibacterium acnes treatment diminishes CD4+ NK1.1+ $T$ cells but induces type I T cells in the liver by induction of IL-12 and IL-18 production from Kupffer cells. J Immunol, 1997. 159(1): p. 97-106.

177. Stoll, S., et al., Production of IL-18 (IFN-gamma-inducing factor) messenger RNA and functional protein by murine keratinocytes. J Immunol, 1997. 159(1): p. 298-302.

178. Mehta, V.B., J. Hart, and M.D. Wewers, ATP-stimulated release of interleukin (IL)-1beta and IL-18 requires priming by lipopolysaccharide and is independent of caspase-1 cleavage. J Biol Chem, 2001. 276(6): p. 3820-6.

179. O'Neill, L.A., D. Golenbock, and A.G. Bowie, The history of Toll-like receptors redefining innate immunity. Nat Rev Immunol, 2013. 13(6): p. 453-60.

180. Ivashkiv, L.B. and L.T. Donlin, Regulation of type I interferon responses. Nat Rev Immunol, 2014. 14(1): p. 36-49.

181. Honda, K. and T. Taniguchi, IRFs: master regulators of signalling by Toll-like receptors and cytosolic pattern-recognition receptors. Nat Rev Immunol, 2006. 6(9): p. 644-58.

182. Schmidt, R.L. and L.L. Lenz, Distinct licensing of IL-18 and IL-1beta secretion in response to NLRP3 inflammasome activation. PLoS One, 2012. 7(9): p. e45186.

183. Zaki, M.H., M. Lamkanfi, and T.D. Kanneganti, The Nlrp3 inflammasome: contributions to intestinal homeostasis. Trends Immunol, 2011. 32(4): p. 171-9.

184. Katakura, K., et al., Toll-like receptor 9-induced type I IFN protects mice from experimental colitis. J Clin Invest, 2005. 115(3): p. 695-702.

185. Ahn, J., H. Konno, and G.N. Barber, Diverse roles of STING-dependent signaling on the development of cancer. Oncogene, 2015. 34(41): p. 5302-8. 
186. Schott, J., et al., Translational regulation of specific mRNAs controls feedback inhibition and survival during macrophage activation. PLoS Genet, 2014. 10(6): p. e1004368.

187. Eldridge, M.J., et al., The Atypical Ubiquitin E2 Conjugase UBE2L3 Is an Indirect Caspase-1 Target and Controls IL-1beta Secretion by Inflammasomes. Cell Rep, 2017. 18(5): p. 1285-1297.

188. Giegerich, A.K., et al., Autophagy-dependent PELI3 degradation inhibits proinflammatory IL1B expression. Autophagy, 2014. 10(11): p. 1937-52.

189. Harris, J., et al., Autophagy controls IL-1beta secretion by targeting pro-IL-1beta for degradation. J Biol Chem, 2011. 286(11): p. 9587-97.

190. Duong, B.H., et al., A20 restricts ubiquitination of pro-interleukin-1beta protein complexes and suppresses NLRP3 inflammasome activity. Immunity, 2015. 42(1): p. 55-67.

191. Radwan, M., et al., Tyrosine kinase 2 controls IL-1ss production at the translational level. J Immunol, 2010. 185(6): p. 3544-53.

192. Guarda, G., et al., Type I interferon inhibits interleukin-1 production and inflammasome activation. Immunity, 2011. 34(2): p. 213-23.

193. Kim, M.L., et al., Aberrant actin depolymerization triggers the pyrin inflammasome and autoinflammatory disease that is dependent on IL-18, not ILIbeta. J Exp Med, 2015. 212(6): p. 927-38.

194. Sharma, D., et al., IL-1beta and Caspase-1 Drive Autoinflammatory Disease Independently of IL-1 alpha or Caspase-8 in a Mouse Model of Familial Mediterranean Fever. Am J Pathol, 2017. 187(2): p. 236-244.

195. Canna, S.W., et al., An activating NLRC4 inflammasome mutation causes autoinflammation with recurrent macrophage activation syndrome. Nat Genet, 2014. 46(10): p. 1140-6.

196. Canna, S.W., et al., Life-threatening NLRC4-associated hyperinflammation successfully treated with IL-18 inhibition. J Allergy Clin Immunol, 2016.

197. Xia, T., et al., Deregulation of STING Signaling in Colorectal Carcinoma Constrains DNA Damage Responses and Correlates With Tumorigenesis. Cell Rep, 2016. 14(2): p. 282-97.

198. Song, S., et al., Decreased expression of STING predicts poor prognosis in patients with gastric cancer. Sci Rep, 2017. 7: p. 39858.

199. Woo, S.R., et al., STING-dependent cytosolic DNA sensing mediates innate immune recognition of immunogenic tumors. Immunity, 2014. 41(5): p. 830-42.

200. Deng, L., et al., STING-Dependent Cytosolic DNA Sensing Promotes RadiationInduced Type I Interferon-Dependent Antitumor Immunity in Immunogenic Tumors. Immunity, 2014. 41(5): p. 843-52.

201. Chandra, D., et al., STING ligand c-di-GMP improves cancer vaccination against metastatic breast cancer. Cancer Immunol Res, 2014. 2(9): p. 901-10.

202. Ohkuri, T., et al., STING contributes to antiglioma immunity via triggering type I IFN signals in the tumor microenvironment. Cancer Immunol Res, 2014. 2(12): $\mathrm{p}$. 1199-208.

203. Corrales, L., et al., Direct Activation of STING in the Tumor Microenvironment Leads to Potent and Systemic Tumor Regression and Immunity. Cell Rep, 2015. 11(7): p. 1018-30. 
204. Fu, J., et al., STING agonist formulated cancer vaccines can cure established tumors resistant to PD-1 blockade. Sci Transl Med, 2015. 7(283): p. 283ra52.

205. Demaria, O., et al., STING activation of tumor endothelial cells initiates spontaneous and therapeutic antitumor immunity. Proc Natl Acad Sci U S A, 2015. 112(50): p. 15408-13.

206. Wilson, J.E., et al., Inflammasome-independent role of AIM2 in suppressing colon tumorigenesis via DNA-PK and Akt. Nat Med, 2015. 21(8): p. 906-13.

207. Brydges, S.D., et al., Divergence of IL-1, IL-18, and cell death in NLRP3 inflammasomopathies. J Clin Invest, 2013. 123(11): p. 4695-705.

208. Naik, S., et al., Inflammatory memory sensitizes skin epithelial stem cells to tissue damage. Nature, 2017. 550(7677): p. 475-480.

209. Friedman, R.M., Clinical uses of interferons. Br J Clin Pharmacol, 2008. 65(2): p. $158-62$.

210. Kuriakose, T., et al., ZBP1/DAI is an innate sensor of influenza virus triggering the NLRP3 inflammasome and programmed cell death pathways. Sci Immunol, 2016. 1(2).

211. Lee, A.J., et al., Inflammatory monocytes require type I interferon receptor signaling to activate NK cells via IL-18 during a mucosal viral infection. J Exp Med, 2017. 214(4): p. 1153-1167. 


\section{VITA}

Qifan Zhu was born in Nanjing, China in the summer of 1990. He received his Bachelor of Science from Huazhong University of Science and Technology in Wuhan, China, with a major in Bioscience. He moved to the United States and started his doctoral study in the Integrated Biomedical Sciences Program at the University of Tennessee Health Science Center in 2012. He is expected to receive his Ph.D. degree in December of 2018 . 